University of Louisville ThinkIR: The University of Louisville's Institutional Repository

Electronic Theses and Dissertations

$8-2013$

\title{
The retina-prosthesis interface in two rat models of retinitis pigmentosa and functional changes associated with photoreceptor loss.
}

James William Fransen

University of Louisville

Follow this and additional works at: https://ir.library.louisville.edu/etd

\section{Recommended Citation}

Fransen, James William, "The retina-prosthesis interface in two rat models of retinitis pigmentosa and functional changes associated with photoreceptor loss." (2013). Electronic Theses and Dissertations. Paper 454.

https://doi.org/10.18297/etd/454

This Doctoral Dissertation is brought to you for free and open access by ThinkIR: The University of Louisville's Institutional Repository. It has been accepted for inclusion in Electronic Theses and Dissertations by an authorized administrator of ThinkIR: The University of Louisville's Institutional Repository. This title appears here courtesy of the author, who has retained all other copyrights. For more information, please contact thinkir@louisville.edu. 


\title{
THE RETINA-PROSTHESIS INTERFACE IN TWO RAT MODELS OF RETINITIS PIGMENTOSA AND FUNCTIONAL CHANGES ASSOCIATED WITH PHOTORECEPTOR LOSS
}

\author{
By \\ James William Fransen \\ B.S University of Illinois, 2004 \\ M.S. Northern Illinois University, 2009

\begin{abstract}
A Dissertation
Submitted to the Faculty of the

School of Medicine of the University of Louisville

In Partial Fulfillment of the Requirements

For the Degree of
\end{abstract} \\ Doctor of Philosophy \\ Department of Anatomical Sciences and Neurobiology \\ University of Louisville \\ Louisville, KY
}

August 2013 
Copyright 2013 by James William Fransen

All rights reserved. 

THE RETINA-PROSTHESIS INTERFACE IN TWO RAT MODELS OF RETINITIS PIGMENTOSA AND FUNCTIONAL CHANGES ASSOCIATED WITH PHOTORECEPTOR LOSS

\section{By}

James W. Fransen

B.S. University of Illinois, 2004

M.S. Northern Illinois University, 2009

A Dissertation Approved On

June $28^{\text {th }}, 2013$

by the following Dissertation Committee

Maureen McCall, Ph.D.

Martha Bickford, Ph.D.

Paul DeMarco, Ph.D.

Ronald Gregg, Ph.D.

Robert Lundy, Ph.D. 


\section{ACKNOWLEDGEMENTS}

I would be remiss if I did not acknowledge the people that have helped me complete this work as none of this would have been possible on my own. Thank you to all my lab members for your help and support. It has been a pleasure working with all of you and your various personalities have made the McCall lab a joy to be a part of. Thank you to my committee members, Dr. Bickford, Dr. Demarco, Dr. Gregg, and Dr. Lundy. Your comments and encouragement towards me and my work have been helpful beyond words. Thank you to my beautiful fiancée, Katie Heath. Your patience and love have helped me through the rough days when the weight of the world seems to bear down. Your smile always seemed to lighten the load. Thank you to my parents who always showed me love and open arms. I'm not sure anyone in the world has a better set of parents than I have and I can only hope to make you proud. Finally but certainly not least of all, thank you to my mentor Dr. Maureen McCall. I still remember first stopping by your office to discuss doing a rotation in the lab. Little did I know what I was signing up for! But in all seriousness, it has been an intense, but very enjoyable, learning process for the last 4 years. I have gained more knowledge by being a part of your lab than I had in all my previous years in college. You seemed to know when to push me and when I just needed some encouragement. Your guidance has been invaluable to me as a scientist and I know I will always be able to come to you for advice no matter what stage of my career I am at. Thank you. 


\title{
ABSTRACT \\ THE RETINA-PROSTHESIS INTERFACE IN TWO RAT MODELS OF RETINITIS PIGMENTOSA AND FUNCTIONAL CHANGES ASSOCIATED WITH PHOTORECEPTOR LOSS
}

\author{
James W. Fransen \\ June $28^{\text {th }}, 2013$
}

With increasingly successful clinical trials, the use of prosthetic devices to replace the function of photoreceptors has now become a viable option to treat degenerative diseases of the retina, including age-related macular degeneration (AMD) and retinitis pigmentosa (RP). Using prosthetics, patients have demonstrated the ability to read large text, recognize small objects, and navigate. While promising, this represents coarse visual function, with the highest visual acuity equivalent to $20 / 560$, still below the legal definition of blindness, 20/200. To investigate the benefit of more sophisticated designs, I implanted two types of photovoltaic prosthetic devices in two models of RP, Tg S334ter-3 and Tg P23H1 rats. The performance of a new bipolar photovoltaic array (bPVA) design with local ground returns was compared to a monopolar photovoltaic array (mPVA) with a single ground return. Regardless of implant design, stimulation parameters at threshold were at least 1.5 orders of magnitude below safety limits. Age and duration of implantation had no significant effect on stimulation thresholds while threshold increased inversely to pixel size. 
In addition to treating the symptoms of these diseases, a better understanding of the functional consequences of degeneration will help guide future treatment strategies. I examined changes in visual function in the retina and the superior colliculus (SC). I found profound changes in visual function in both models. First, the ON pathway is preferentially lost due to a shift from equal numbers of ON and OFF ganglion cells in the retina and ON dominated responses in the SC to OFF dominated responses in both. In the $\mathrm{Tg} \mathrm{P} 23 \mathrm{H}-1$ rat, this shift is progressive with age, while in the $\operatorname{Tg}$ S334ter-3 this shift is evident early in life. In late stages of the disease, there is little response from the ON pathway. In animals implanted with bPVA devices, there was a significant change in the response based on the location of the prosthesis, with a preservation of the ON pathway in areas directly under the influence of the prosthesis. Either the presence of the device or basal electrical activity may induce a trophic influence that attenuates this change in visual function. 


\section{TABLE OF CONTENTS}

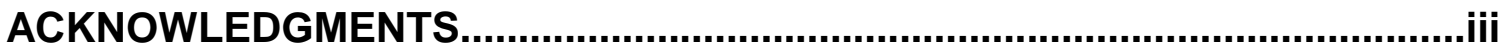

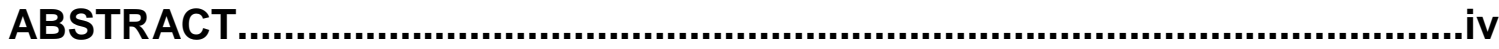

LIST OF FIGURES...........................................................................................viii

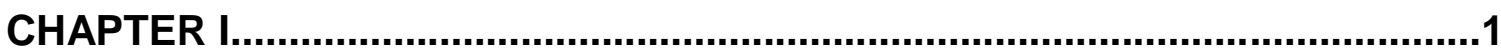

INTRODUCTION AND GENERAL BACKGROUND INFORMATION.................1

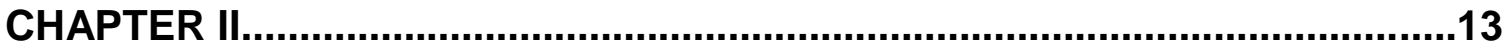

COMPARISON OF THE RETINA-PROSTHESIS INTERFACE OF TWO PHOTOVOLTAIC ARRAY DESIGNS IMPLANTED IN A RAT MODEL OF RETINITIS PIGMENTOSA

Introduction..............................................................................13

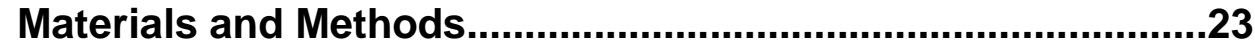

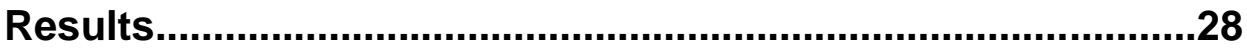

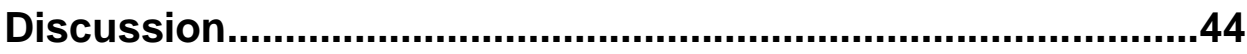

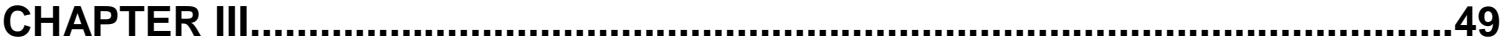

FUNCTIONAL ASSESSMENT OF TWO RAT MODELS OF RETINITIS PIGMENTOSA INDICATE A PREFERENTIAL LOSS OF THE ON PATHWAY DURING THE DEGENERATIVE PROCESS

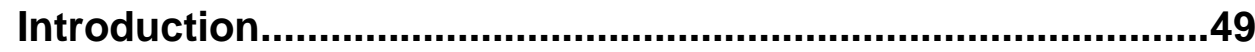

Materials and Methods.........................................................52

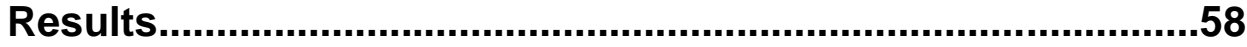

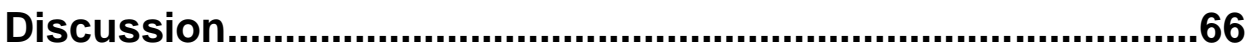

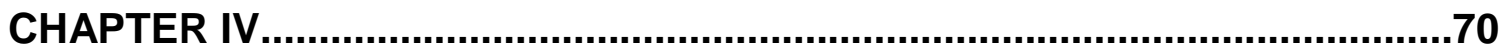

IMPLANTATION OF RETINAL PROSTHETIC DEVICES IMPARTS A

FUNCTIONAL NEUROPROTECTIVE EFFECT IN REGIONS OF THE RETINA UNDER THE INFLUENCE OF THE PROSTHETIC DEVICE

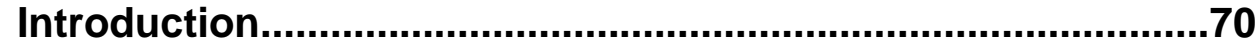

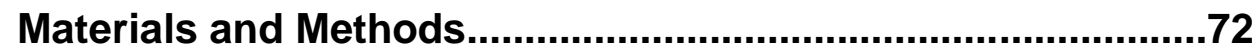




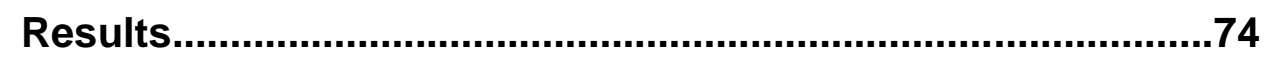

Discussion..........................................................................82

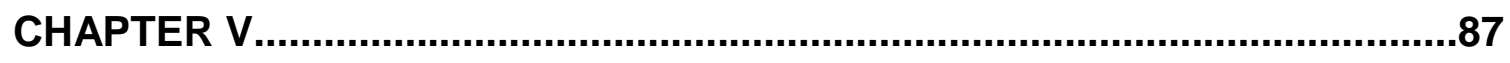

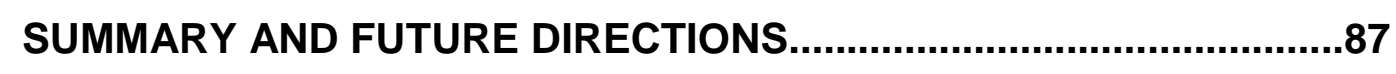

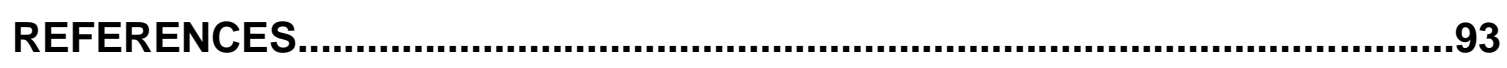

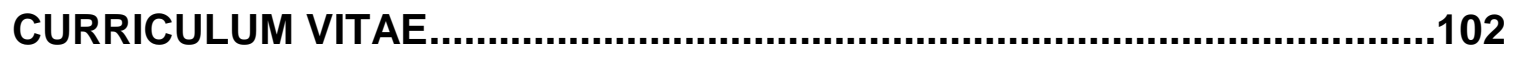




\section{LIST OF FIGURES}

Figure 1. Laminar structure of the vertebrate retina......................................3

Figure 2. Photoreceptor structure and signaling cascade............................5

Figure 3. The rod signaling pathway................................................................

Figure 4. Retinal projections to the dorsal surface of the left SC..................9

Figure 5. Normal and RP patient fundus images...........................................10

Figure 6. Tg photoreceptor degeneration rates............................................12

Figure 7. Intraocular implantation approaches...............................................17

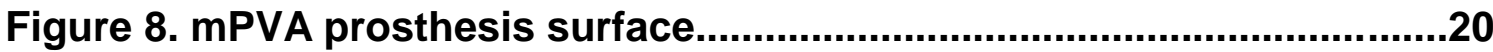

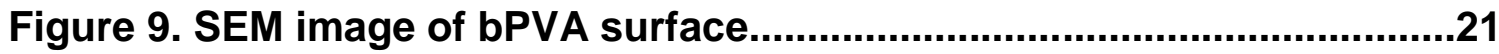

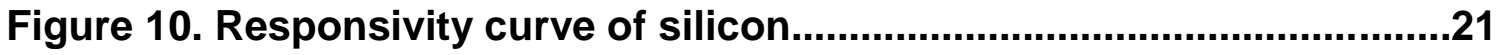

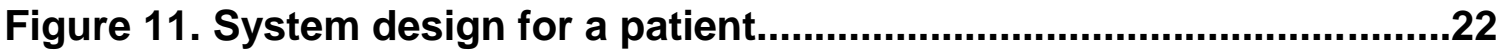

Figure 12. In vivo imaging of prosthetic device............................................25

Figure 13. Spectral sensitivity of rat cones...............................................26

Figure 14. PSTH and rater plot of prosthesis-evoked responses.................29

Figure 15. Visually-evoked vs. prosthesis-evoked responses.......................30

Figure 16. Injection of synaptic blockers eliminates prosthesis-evoked

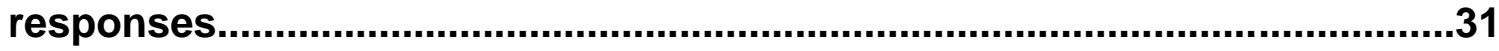

Figure 17. Representative fundus image and SC map...................................34

Figure 18. Measurement of the predicted and actual location of SC

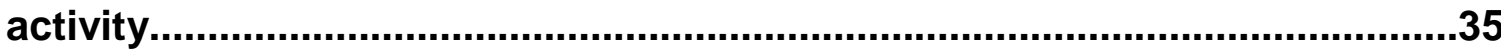

Figure 19. Predicted vs. actual location of SC activity.................................36

Figure 20. Intensity-duration curves for implantation age and duration......38

Figure 21. Intensity-duration curves for pixel size and device type.............39

Figure 22. Normalized response as a function of pulse duration.................40

Figure 23. Stimulus locations for the two device types................................41

Figure 24. Modulated vs. non-modulated response examples......................42 
Figure 25. Percentage of devices with modulated responses.....................43

Figure 26. The 54 recording sites across the dorsal surface of the SC......53

Figure 27. Raw trace, PSTH, and wave form examples...............................55

Figure 28. Typical visually-evoked responses in WT and Tg rats...............59

Figure 29. ON and OFF total response in Tg P23H-1 rats vs. WT................59

Figure 30. Duration and amplitude of Tg P23H-1 rats vs. WT......................60

Figure 31. ON and OFF total response in Tg S334ter-3 rats vs. WT.............61

Figure 32. ON-OFF index for Tg S334ter-3 rats vs. WT.................................62

Figure 33. Cell class profile for $\mathrm{P} 35$ and $\mathrm{P} 300 \mathrm{Tg} \mathrm{P} 23 \mathrm{H}-1$ rats vs. WT........64

Figure 34. Average FFT peak of P300 Tg P23H-1 RGC classes....................65

Figure 35. Schematic of IR, Near, and Far response sites.............................74

Figure 36. Example SC topographic maps from implanted Tg P23H-1

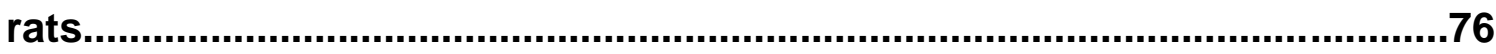

Figure 37. ON-OFF index values in implanted Tg $\mathrm{P} 23 \mathrm{H}-1$ rats......................76

Figure 38. ON and OFF peak response and duration in implanted Tg

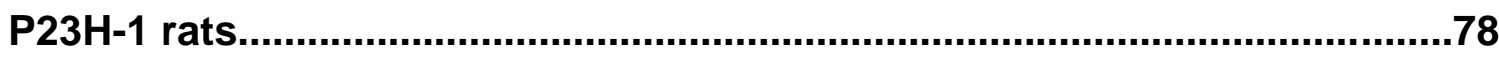

Figure 39. Spontaneous SC activity in implanted Tg P23H-1 rats.................79

Figure 40. ON-OFF index values in implanted Tg S334ter-3 rats..................80

Figure 41. ON and OFF peak response and duration in implanted Tg

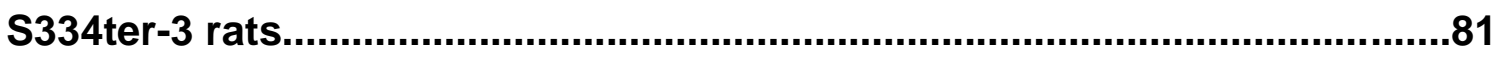

Figure 42. Spontaneous SC activity in implanted Tg S334gter-3 rats..........82 


\section{CHAPTER I}

\section{INTRODUCTION AND GENERAL BACKGROUND INFORMATION}

Regardless of species, the need for an organism to gather information about its environment is universal. Tactile, chemical, and photic stimuli have all driven the evolution of different sensory modalities to best detect stimuli contained in an organism's natural environment. The ability to detect light, food sources and environmental danger led to the development of visual systems early in evolutionary history. Early vision was likely very simple, consisting of little more than aggregations of proteins in bacteria that drove phototaxis. These proteins were able to change conformation in response to the presence or absence of light. Providing feedback to the organism, this change could then be used to drive the bacterium towards or away from a source of light depending on the needs of the organism (Suzuki et al., 2010).This rudimentary system evolved

along with the diversity of life over billions of years to provide more thorough and sophisticated visual information regarding the environment. The study of the visual system has revealed an exquisitely tuned system for collecting and interpreting visual signals in the specific environment of a species. Visual systems have evolved to the circadian cycle, food source, predatory species, etc. 
contained in an organism's specific environment. For example, bees (Skorupski and Chittka, 2010) and mice (Jacobs et al., 1991) can detect light in the ultraviolet (UV) spectrum, below the visible wavelengths of humans. This sensitivity to UV wavelengths increases the bee's ability to find specific food sources in the form of flowers that reflect UV light. The visible spectrum for humans is at least partially tied to food source as well. In a similar fashion, it is hypothesized that humans developed color perception at least in part to better assess the ripeness of fruits and vegetables, which is related to its nutritional value (Lucas et al., 2003). These are but a small fraction of examples that could be used to highlight the importance of the visual system to most organisms. Given the importance of environmental stimuli to an organism, deficits in any sensory system can be devastating. Within the visual system, mutations in the proteins that begin the process of phototransduction can lead to debilitating visual deficits or even complete blindness. The general goals of this dissertation are to assess a potential treatment for blinding eye diseases in the form of retinal prosthetic devices, describe functional changes that occur in the retina and its first synapse as a result of photoreceptor degenerative processes, and address the possibility that implantation of a prosthetic device may impart a neuroprotective effect on the function of these retinas. 


\section{The Visual System and Phototransduction}

\section{Retinal Structure and Cell Classes}

The retina is a neural structure that

contains five general cell classes. These

cells form a laminar structure in which three

cellular layers alternate with two plexiform

(synaptic) layers (Figure 1). The

photoreceptor cell bodies form the first

cellular layer, the outer nuclear layer (ONL).

In the outer plexiform layer (OPL),

photoreceptors synapse with cells of the

inner nuclear layer (INL), namely the

bipolar cells (BCs) and the horizontal cells

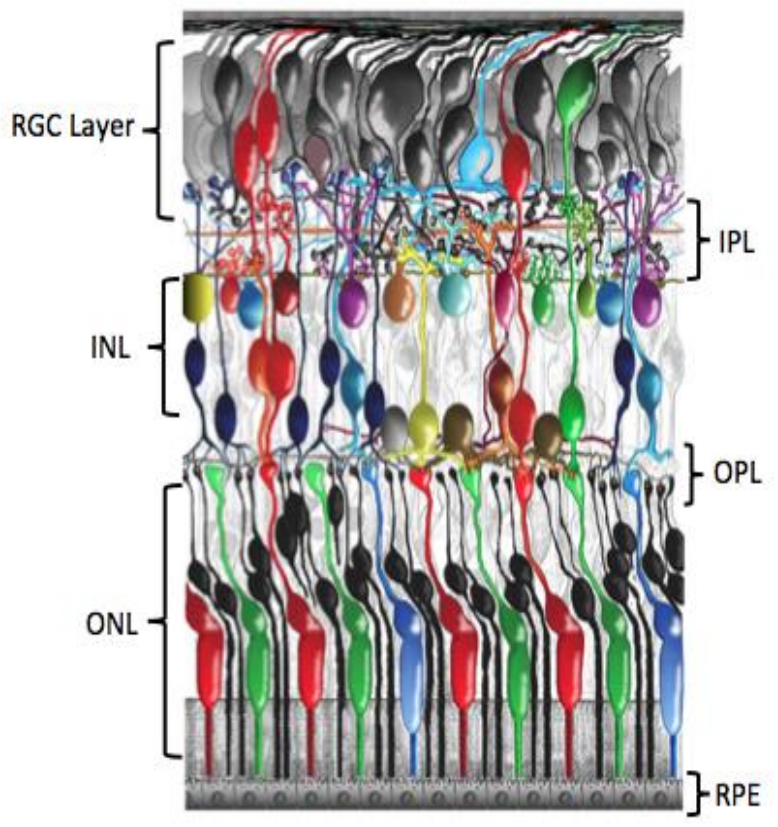

Figure 1. Laminar structure of the vertebrate retina. Modified from webvision.med.utah.edu. (HCs), which modulate the photoreceptor

output. The BCs provide the excitatory drive to amacrine cells (ACs), also located in the INL and both BCs and ACs synapse onto retinal ganglion cells (RGCs) in the inner plexiform layer (IPL). RGCs are projection neurons whose axons exit the back of the eye to form the optic nerve and transmit signals to the visual centers of the brain.

\section{Phototransduction}

Phototransduction is the conversion of light into an electrical signal within the photoreceptor. Photosensitivity within photoreceptors arises due to the presence 
of photopigments, called opsins, in the outer segment (OS) (Figure 2). In the dark, cGMP-gated cation channels in the photoreceptor outer segments are in an open state. Current flows into the outer segment (the 'dark current'), the photoreceptors depolarize and in the dark release glutamate. In the inactive state, opsin proteins are bound to a trimeric $\mathrm{g}$-protein, called transducin, which is composed of an alpha, beta, and gamma subunit. In the dark, GDP is attached to the alpha subunit and keeps it bound to the beta and gamma transducin subunits. When a photoreceptor absorbs a photon, 11-cis retinal changes conformation to all-trans form and activates rhodopsin. Once rhodopsin is activated, the GDP bound to the alpha transducin subunit is phosphorylated to GTP and the alpha subunit is released. The now activated alpha subunit is free to bind and activate cGMP phosphodiesterase. This enzyme catalyzes the hydrolysis of cGMP to a non-cyclic form, with the decrease in cGMP closing the cation channels and hyperpolarizes the photoreceptor. This decreases glutamate release in a graded fashion, proportional to the light energy (Figure 2). 
A.

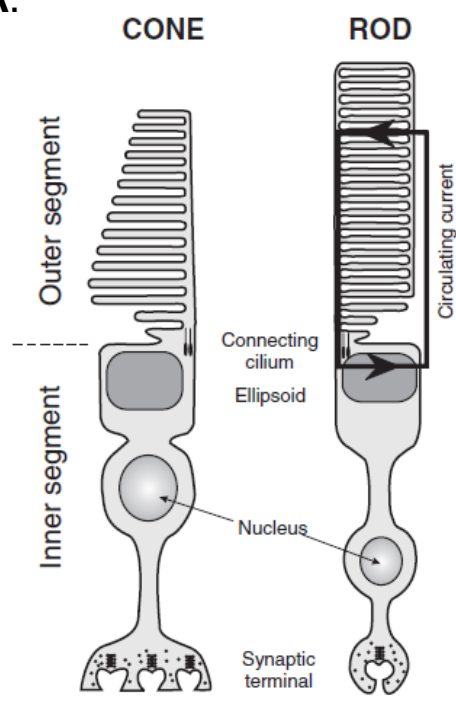

B.

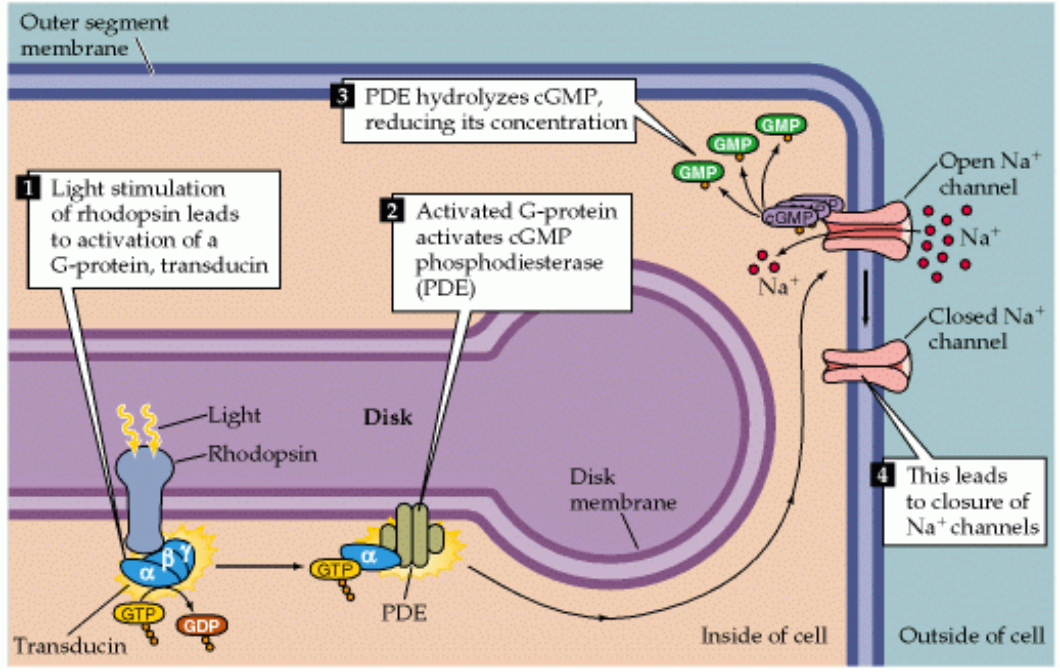

Figure 2. A. Structure of cone and rod photoreceptors. B. The elements of the phototransduction cascade. Adapted from: Left: The Visual Neurosciences (Chalupa and Werner). Right: Purves et al., 2001.

There are two classic types of retinal photoreceptors: rods and cones.

Because of their differential sensitivity to light, these two types give the retina the ability to work over a luminance range of about 12 orders of magnitude. The rods are more sensitive than the cones, with the ability of a rod to respond to a single absorbed photon (Baylor et al., 1979). To achieve high sensitivity, rods pool their signals at subsequent synapses, but the consequence is poor spatial resolution under rod driven vision. Therefore, rods are utilized under low light conditions when the ability to detect light and movement are more important than resolution. Cones are active during higher ambient light conditions and their nearly "one to one" synaptic connections in the fovea result in high acuity. Because cones can express one of two or three opsins (depending on the species) with different spectral sensitivities, they initiate color perception. 


\section{Normal Retinal Signaling}

The photoreceptor's reduction in glutamate release causes a change in the polarization of their postsynaptic partners, the BCs and HCs. The sign of the polarization depends on the type of glutamate receptor expressed by the HCs and the ten morphological types of BC. There are four morphological types of OFF cone BCs which express ionotropic glutamate receptors (iGluRs), which are comprised of a cation channel with a glutamate receptor binding site. iGluRs open when glutamate binds and depolarize then quickly desensitize due to the properties of the channel. When glutamate concentrations in the synapse are low (in the light), the iGluR channel is closed, similar to the closure of the channels in the photoreceptor. This means that the OFF BCs conserve the sign of the photoreceptor signal (hyperpolarization) to light and depolarize at the onset of a luminance decrement.

There are five types of $\mathrm{ON}$ cone $\mathrm{BCs}$ and one type of $\mathrm{ON}$ rod $\mathrm{BC}$ that express metabotropic glutamate receptor type 6 (mGluR6), which is a G-protein coupled receptor. When glutamate concentrations are low in the synapse, a G-protein cascade is inhibited and the non-specific cation channel, TRPM1, is opened. In the dark when glutamate concentrations are high, the G-protein cascade is initiated which closes the TRPM1 channel. In this way, ON BCs invert the sign of the photoreceptor signal; when the photoreceptor is hyperpolarized, the BC will depolarize. At this first synapse, the opposite $\mathrm{BC}$ responses create the $\mathrm{ON}$ (BCs with mGluRs) and OFF (BCs with iGluRs) parallel signaling pathways within the 
retina. At this synapse, horizontal cells use feedback inhibition to modulate photoreceptor output and to generate an inhibitory surround in the BCs.

Within the cone pathway, photoreceptors signal to ON and OFF cone BCs, which synapse directly onto RGCs and generate an excitatory input via glutamate release. Rod BCs are ON BCs, but do not directly synapse onto RGCs. Instead, they utilize a narrow field bistratified $A C$, the All $A C$, as an intermediary to provide input to both the ON and OFF cone pathways but the All connects to each with a different mechanism. The All AC connects to $\mathrm{ON}$ cone BCs through a gap junction, creating a signal of the same sign as the presynaptic input. The All connects to OFF cone BCs or OFF RGCs via an inhibitory synapse, releasing glycine to inhibit the OFF pathway (Figure 3). With these connections the All AC is able to convey information to both pathways: excitation to the ON pathway and inhibition to the OFF pathway during rod driven vision. BCs signal to RGCs via glutamate and ACs modulate the $\mathrm{BC}$ signal via a number of inhibitory mechanisms and using the neurotransmitters GABA or glycine. 


\section{The Superior Colliculus}

The superior colliculus (SC) is a subcortical structure that is a direct synaptic target of RGC axons. In mice, it is estimated that at least $70 \%$ of RGCs make synaptic connections with cells in the SC (May, 2006). The SC is organized as a laminar structure. The superficial (dorsal) layers receive synaptic input primarily ( 95-98\%) from contralateral RGC axons, whereas the more ventral layers process other sensory modalities or integrate multi-sensory information (May, 2006). The most likely function of the SC is to direct attention towards a novel or moving stimulus (Robinson, 1972). For most animals, this plays a vital role in the ability to spot prey or avoid predators. The SC provides input to the lateral geniculate nucleus (LGN) (Hoffman, 1973), another direct synaptic target of RGC axons, and receives input from the primary visual cortex (Lund, 1964). These connections lead to the hypothesis that the SC likely has a modulatory role for image formation and is modulated by the input from $\mathrm{V} 1$, although the specific functions of these connections are not as well understood.

The axons of the RGCs form a topographic map of the retina on the dorsal surface of the SC (Figure 4). This means adjacent RGCs within the retina synapse at adjacent points across the SC dorsal layers (Drager and Hubel, 1976). This spatial information about regional changes within the retina also can be used to localize the area of the retina that is stimulated by a prosthetic device. 


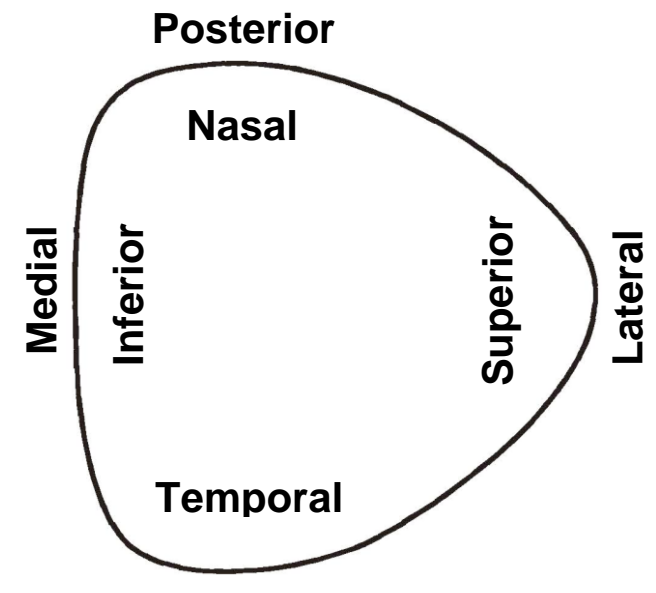

Figure 4. Retinal projections to the dorsal surface of the left SC. Labels inside the diagram represent the projections from RGCs. Labels outside the diagram represent the spatial layout of the SC surface.

\section{Retinitis Pigmentosa and Rat Models of RP}

\section{Causes of RP}

Retinitis pigmentosa is a group of disorders characterized by the loss of rod photoreceptors and in many cases, subsequent loss of cone photoreceptors. The majority of cases of RP result from mutations in rhodopsin or its signaling cascade. This can lead to difficulty with peripheral vision or low light vision, such as driving at night, which typically are the first symptoms that push patients to see their doctor. Upon examination, the typical signs of RP in the fundus include bone spicule formation, arteriolar atrophy and optic disc pallor, which are accompanied by a reduced electroretinogram (ERG) response (Fishman et al., 1988; Figure 5). 


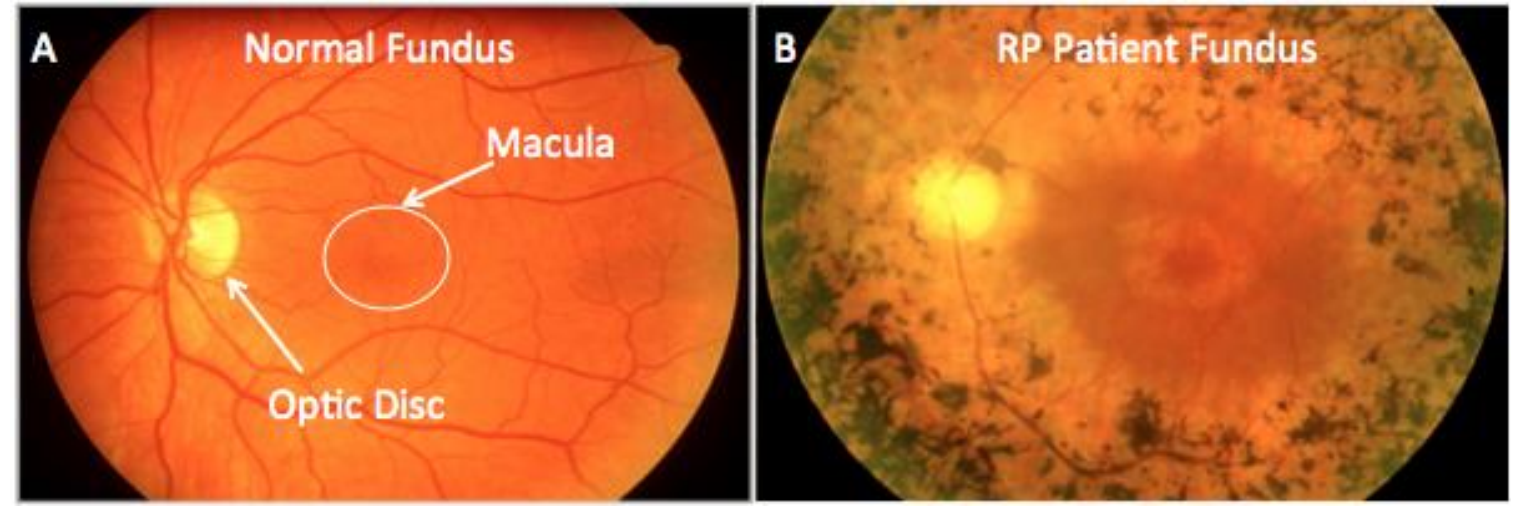

Figure 5. A: Fundus image of normal retina. B: Fundus image of advanced RP patient, note the appearance of bone spicules, optic disc pallor, and blood vessel atrophy. Modified from webvision.med.utah.edu

While many patients present with similar signs and symptoms, the genetic cause varies extensively. Mutations in a number of different genes cause RP, for example, mutations in rhodopsin (Dryja et al., 1990), in genes expressed by the retina pigment epithelium (RPE) (Thompson et al., 2002), in photoreceptor transcription factors (Martinez-Gimeno et al., 2001), and in accessory proteins associated with photoreceptors (Kimberling et al., 1990). Even with the reduced price and increased access of genetic testing to the public, up to $40 \%$ of genetic causes of RP remain unknown (Hartong et al., 2006). In addition, RP is inherited in multiple ways; some are autosomal dominant (ADRP), others autosomal recessive or X-linked. This heterogeneity in mutation and inheritance means that any potential genetic therapy approach is unlikely to be universally applicable. As a consequence, other therapeutic approaches are being developed to treat RP. They include: neuroprotection to delay degeneration, cellular therapies with the hope of replacing lost photoreceptors, optogenetic expression of bacterial 
channel opsins to replace lost photoreceptor function and finally, prosthetic devices (including retinal, optic nerve, LGN and cortical).

\section{Transgenic (Tg) P23H-1 and S334ter-3 Rat Models of RP}

Once the genetic causes of RP started to be defined, multiple animal models of RP were found or created using molecular manipulation. These include mice (Olsson et al., 1992, McNally et al., 2002, Price et al., 2011), rats (Bok and Hall 1971, Lewin et al., 1998, Liu et al., 1999), rabbits (Kondo et al., 2009) and pigs (Petters et al., 1997, Ross et al., 2012). Two Tg rat models (with multiple lines in each) that express rhodopsin mutations, $\mathrm{P} 23 \mathrm{H}$ and $\mathrm{S} 334$ ter have been widely used. The $\mathrm{Tg} \mathrm{P} 23 \mathrm{H}-1$ rat results from a single point mutation that alters the $23^{\text {rd }}$ amino acid residue in rhodopsin from a proline to a histidine in the mutated protein (Lewin et al., 1998). This amino acid change prevents the mutant rhodopsin from folding properly. Deficiencies in the ubiquitin protease system associated with these mutations lead to the accumulation of the mutant protein in the photoreceptor cell body and cause apoptosis (Illing et al., 2002). The $\mathrm{P} 23 \mathrm{H}$ mutation is the most common form of ADRP in North America, accounting for $12 \%$ of all cases (Dryja et al., 1990).

The Tg S334ter-3 rat results from a point mutation that causes an early stop codon at the $334^{\text {th }}$ residue, normally a serine (ucsfeye.net). This change results in the expression of a truncated rhodopsin protein in which 15 amino acids are lost from the C-terminus, which results in the inability to properly traffic rhodopsin to the outer segments (Concepcion et al., 2002). Like mutant P23H 
rhodopsin, this protein also accumulates in the photoreceptor cell body and causes apoptosis (Shinde at al., 2012). Within each model there are multiple lines distinguished by their different rates of degeneration, which most likely arises from positional effects of transgene insertion and differential expression of the transgene. Line number is designated at the end of the name, for example $\mathrm{Tg}$ $\mathrm{P} 23 \mathrm{H}-1$ represents line 1.

Both Tg P23H-1 and Tg S334ter-3 models show initial rod photoreceptor death followed by cone photoreceptor death at later stages, much like the course of human RP. Both models show autosomal dominant inheritance patterns, similar to some human RP patients. These models were chosen, for my experiments in order to contrast and compare a fast (Tg S334ter-3) and a slow degeneration line $(\mathrm{Tg} \mathrm{P} 23 \mathrm{H}-1)$. The rates of degeneration have been measured morphologically using ONL thickness (Figure 6;

ucsfeye.net/mlavailRDratmodels.shtml).

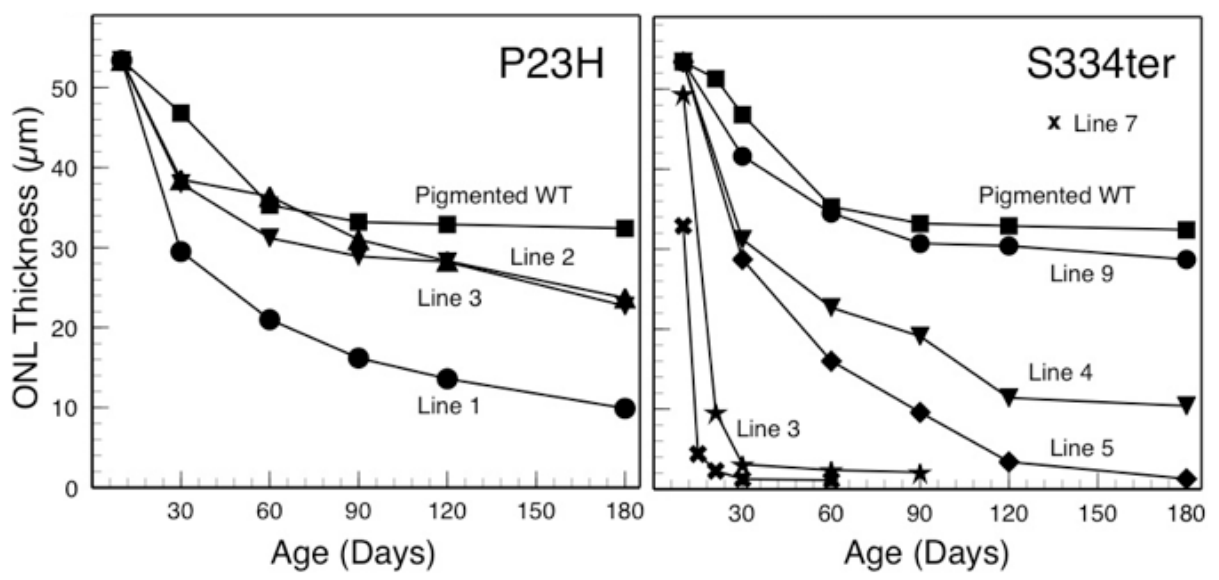

Figure 6. Degeneration rates differ across $\mathrm{Tg} \mathrm{P23H}$ and $\mathrm{Tg}$ S334ter rat lines. Tg P23H line 1 and Tg S334ter line 3 rats were used for my experiments. (Modified from ucsfeye.net/mlavailRDratmodels.shtml) 


\section{CHAPTER II}

\section{COMPARISON OF THE RETINA-PROSTHESIS INTERFACE OF TWO SUBRETINAL PHOTOVOLTAIC ARRAY DESIGNS IN A RAT MODEL OF RETINITIS PIGMENTOSA}

\section{Introduction and Background}

Many blinding eye diseases result from photoreceptor dysfunction and degeneration. When this occurs, vision can be severely impaired and visual perception may be lost completely. The most prevalent degenerative photoreceptor diseases include AMD and RP. Together they affect approximately 1.9 million people in the United States and this number is expected to increase to nearly three million within the next eight years as the baby boom generation continues to age (Friedman et al., 2004). Currently, patients have few treatment options and most therapies only delay disease progression. In AMD and RP, although photoreceptors are lost, most of the other retinal cell classes remain; including BCs, ACs and RGCs. The pathways through the retina initiate visual image formation and send input to all higher visual centers in the brain. Thus, many therapies aim to either replace the cells themselves or replace their function. At present, there is no proven cellular therapy to replace lost 
photoreceptors. Other strategies include optogenetic expression of a bacterial opsin in the remaining BCs and/or RGCs or replacement of photoreceptor function with a prosthetic device.

Retinal neurons use changes in electrical potential to generate chemical signals that transmit information across synapses, thus applied external electrical current can stimulate neurons of the retina (Gebhard, J.W. 1952, Kurosawa 1954). As a potential treatment for the loss of photoreceptors, a retinal prosthetic that responds to light and produces current should be capable of stimulating the $\mathrm{BC} / \mathrm{RGC}$ pathway. A highly proficient prosthetic device will have a high density of electrodes; ideally it will emulate the ratio of electrode to $\mathrm{BC}$ similar to that found between retinal photoreceptors and BCs. For optimal spatial coding, the electrode size should be small but also provide sufficient current to stimulate a small number of BCs over the extent of their dendritic arbor to theoretically increase spatial resolution. Stimulation thresholds should be low and there should be a linear range of current to response to emulate the graded response of the photoreceptor itself. To be of use for a majority of patients, the prosthetic/retina interface should be established regardless of disease progression and should remain intact over a long time period. While devices such as this have been made and tested previously, their design has produced limited spatial discrimination. Towards the goal of a high-resolution prosthetic device, a design has recently been produced to address these spatial limitations (Mathieson et al., 2012). With this new design, current spread is theoretically 
more localized with the idea that it will enhance spatial acuity and the resolution for an implanted patient.

\section{Applied Electrical Stimulation}

The ability of electrical stimulation to reliably elicit light perceptions (phosphenes) in completely blind patients (Humayun et al., 1999) supports the possibility that photoreceptor function can be replaced using implanted prosthetic devices. Direct stimulation of the RGCs results in a 1:1 ratio of pulse to action potential while stimulating the BCs results in a burst of RGC activity (Eikenscheidt et al., 2012). This supports the theory that stimulating through BCs allows the signal to be processed by the retinal circuit, potentially leading to a better approximation of normal vision. Importantly, multiple studies have reported that thresholds are the same when stimulating either through BCs or RGCs directly (O'Hearn et al., 2006, Shah et al., 2006, Shyu et al., 2006). If thresholds are the same regardless of the site of stimulation, then the better site should be the one that best approximates the normal flow of the visual signal.

\section{The Photovoltaic Effect}

Prosthetic devices have multiple designs to create the electrical activity that drives the postsynaptic cells. Most prosthesis designs utilize an external power source to drive the device and deliver current to the retinal cells. An external power supply is particularly useful for devices implanted in parts of the visual system that cannot be directly stimulated by light, such as the optic nerve. When 
a prosthetic device is placed in the eye, it is possible to use light to generate electric current directly from the device in a process known as the photovoltaic effect. This is the same technology that solar panels use to convert sunlight into energy to power our homes and cities. The process relies on the chemical properties of semiconductor materials such as germanium, selenium, gallium, telluride, and the most common material used, silicon. (www.eere.energy.gov). Crystalline forms of these materials absorb light of sufficient energy and release electrons bound in their crystalline lattice to a free conduction energy state. When positive and negative diodes are paired, an electric field is introduced, which generates current flow between them (pveducation.org). The current generated can then be collected, as in solar cells, or in the prosthetic device to stimulate the retina.

\section{Prosthetic Devices and the Visual System}

Multiple areas within the visual system are active areas of research for prosthetic devices: the retina (Chow et al., 2004, DeMarco et al., 2007, Jensen and Rizzo 2008), optic nerve (Veraart et al., 1998), and primary visual cortex (Maynard et al., 1997) are all targets. Currently, there is a debate over the best placement of a prosthetic device and how to stimulate the retina: trans-scleral, suprachoroidal, subretinal or epiretinal (Rettay and Resatz, 2004; Figure 7).

The trans-scleral approach places the prosthesis outside of the eye on the sclera. The current produced spreads through the sclera, choroid and RPE to stimulate the retina. The clear advantage to this placement is a minimally 
invasive surgery with few risks such as infection or surgical damage to the eye or retina. However, there are significant disadvantages to this approach. In particular, much higher current is required to stimulate the retina compared to

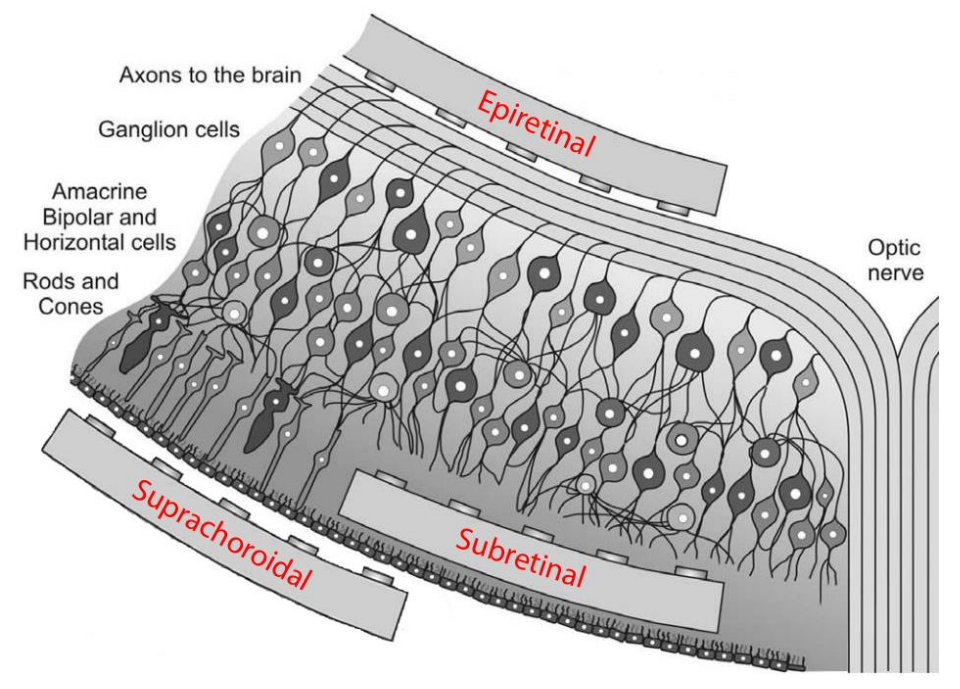

Figure 7. Intraocular implantation approaches for retinal prosthetic devices. Modified from Rattay and Resatz, 2004. intraocular approaches. The

high current levels may

adversely affect the sclera

and other surrounding

tissues. Further, the

resolution achieved is

significantly reduced due to

lateral current spread along

the outside of the eye.

The epiretinal approach

places the prosthesis within the vitreous cavity, close to the RGCs and their

nerve fiber layer (NFL). The advantage is that RGCs and their axons are directly

stimulated, which improves spatial resolution compared to a trans-scleral

approach. A significant disadvantage is that all current epiretinal designs have external power supplies with wires that exit the eye. This creates a permanent open surgical site, which increases the risk for complications, such as corneal erosion and infections that have been reported in the Argus II trial (Humayun et al., 2012). In addition, the wires must be configured to permit the eye and wire to move freely. Improper placement increases stress on these wires and may result 
in catastrophic failure, requiring additional surgery and risk to the patient to replace the damaged unit.

Subretinal and suprachoroidal approaches attempt to stimulate the retina from the photoreceptor side. The advantage of stimulating from the INL side is that it should stimulate cells in the INL to make use of the inherent circuitry within the INL rather than bypassing it and stimulating RGCs directly (Cuenca et al., 2004). Thus, the signal should be modulated before it is transmitted to the RGCs and the rest of the visual system and should result in a better approximation of normal vision. To date the interface between the prosthetic devices and the inner retina has not been empirically tested in vivo. Whether the current is limited to the inner retina or extends to directly stimulate the RGCs remains unknown. In a subretinal approach, the prosthesis is inserted within the subretinal space with the electrodes of the prosthesis in direct contact with the INL. In contrast, in a suprachoroidal approach, the prosthesis is placed on top of the choroid and must pass current through the choroid before reaching the INL. The possible advantage over subretinal placement is the diffusion of nutrients from the choroid may be improved. Although, the work of Pardue and colleagues indicates that the absence of nutrient diffusion through a solid device over 8 weeks is not detrimental to the underlying retinal morphology in RCS rats (another model of $\mathrm{RP}$ ), when implanted long term (27 weeks) in wild-type cats there was a significant loss of photoreceptors directly beneath the device (Pardue et al., 2001, Pardue et al., 2005). Whether function or the site of stimulation is altered over time also has not been addressed. New designs are incorporating pores or 
channels within the device material to permit better nutrient diffusion. Whether this will improve the outcome also has not been tested.

\section{Neuroprotective effects of prosthetic devices and electrical stimulation}

The direct benefits of prosthetic devices may be augmented by neuroprotective effects. Pardue et al. (2005) showed an increased electroretinogram (ERG) b-wave amplitude and photoreceptor preservation in implanted RCS rats in the area of the implant. Demarco et al. (2007) reported a trophic effect in RCS rats implanted with a prosthetic device even when the device was an inactive type (non-current producing). Visual responses were more robust within the area of the implant when compared to control RCS rats. In contrast, the Mertk mouse, which has a similar mutation as the RCS rat, did not show a neuroprotective effect from an implanted mPVA (Mocko et al., 2011). In addition to effects of prosthetic devices, electrical stimulation has been reported to protect axotomized RGCs (Morimoto et al., 2005), preserve photoreceptors ( $\mathrm{Ni}$ et al., 2009, Morimoto et al., 2012) and increase ERG amplitudes (Ni et al 2009). The potential for neuroprotective effects has important implications concerning when patients may optimally benefit from implantation of a prosthetic device. 
The mPVA (ASR) and bPVA Designs

The mPVA retinal prosthetic device was one of the earliest designs used in human clinical studies (Figure 8; Optobionics, Inc., Chow et al., 2004, Chow et al., 2010). In the RCS rat model of RP, DeMarco et al. (2007) were the first to demonstrate the functional efficacy of the MPVA device in a degenerate retina by recording prosthesis-evoked activity in the SC. While these were promising results, the mPVA design is limited by two important factors. First, the solid wafer design limits diffusion of nutrients to the retina directly under the device (Chow et al., 2001) and could impact the preservation of inner retina, exacerbating degeneration. Second, although the mPVA

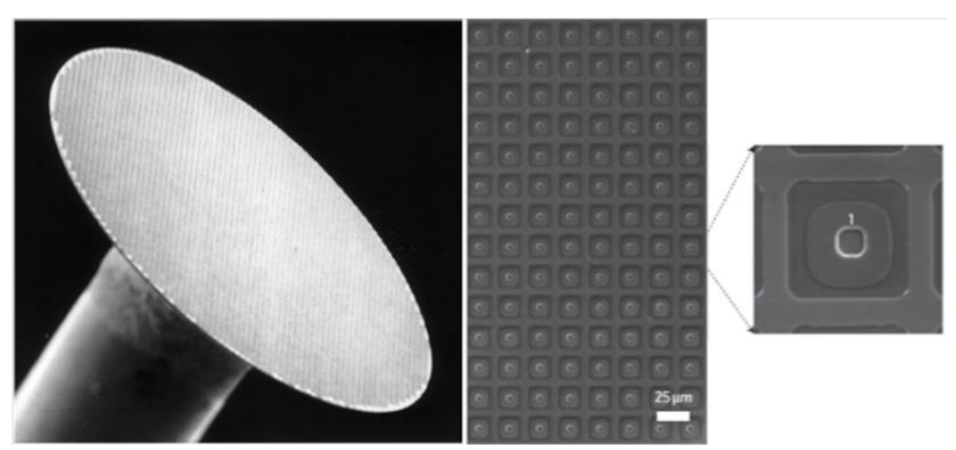

Figure 8. mPVA type prosthesis. Left: The $1 \mathrm{~mm}$ MPVA device. Middle: Expanded view of the electrode array. Right: Magnified view of a single electrode in the array. Modified from Mathieson et al.. 2012.

incorporates hundreds of tiny electrodes (each $10 \mu \mathrm{m}$ ) on its surface that have the potential to generate high acuity vision, its single ground return electrode limits its spatial resolution. With the return electrode located on the opposite side of the electrodes, current must flow around the prosthesis. The current could potentially stimulate any cell along the path it takes, degrading spatial resolution. A new photovoltaic prosthetic design (Figure 9; Mathieson et al., 2012) attempts to address both nutrient diffusion and current flow. First, the new design has $5 \mu \mathrm{m}$ 


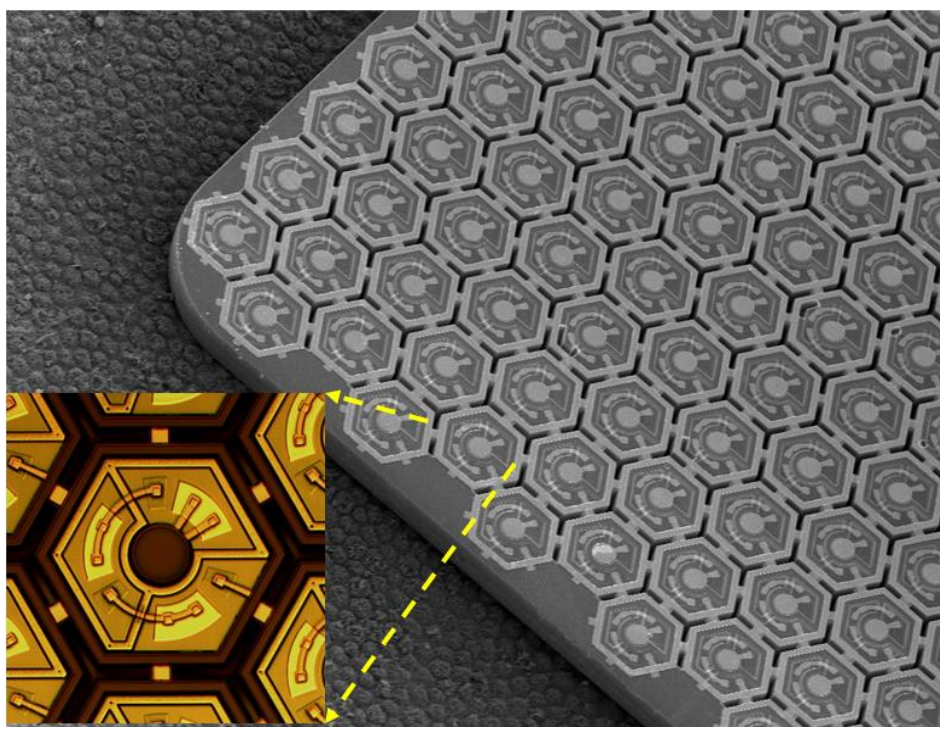

Figure 9. Scanning electron microscopy image of the bPVA prosthesis current generating surface with a magnified single pixel shown in the inset. Modified from Wang et al., 2012. gaps around each electrode

that should increase nutrient

diffusion through the

prosthesis to the retina. The

design also includes a high

pixel-density array with

individual return electrodes

surrounding each pixel.

Secondly, this design uses

a bipolar pulse with a

cathodic and anodic phase,

which has been shown to produce a more consistent response to repeated stimulation than pulses of either type alone (Jensen and Rizzo, 2009). Prototype designs have been produced in three different pixel (electrode) sizes (70, 140 , and $280 \mu \mathrm{m})$.

The intrinsic response properties of silicon make near infrared (NIR) light an ideal stimulus in terms of the responsivity of the array material to wavelength (Figure 10). Essentially, this means the device will create more current for

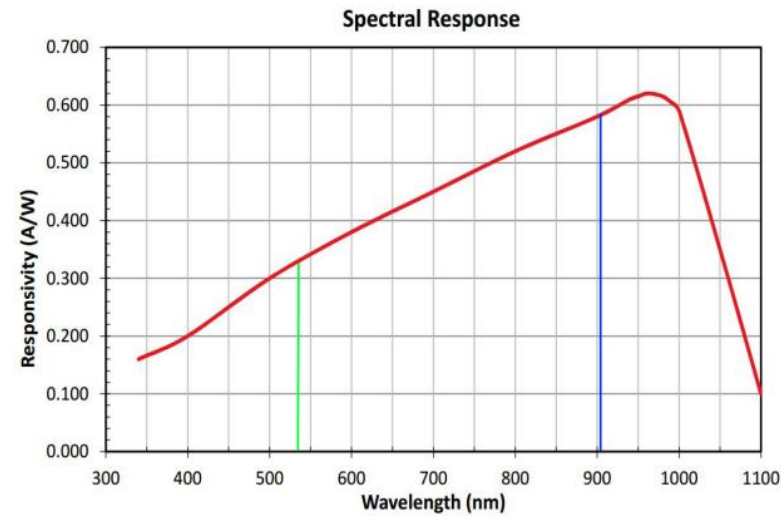

Figure 10. Typical responsivity curve of silicon based optical sensor. The blue line represents the wavelength of the near-infrared laser and the green line represents the visible laser wavelength of the PASCAL system. Modified from www.thorlabs.com 


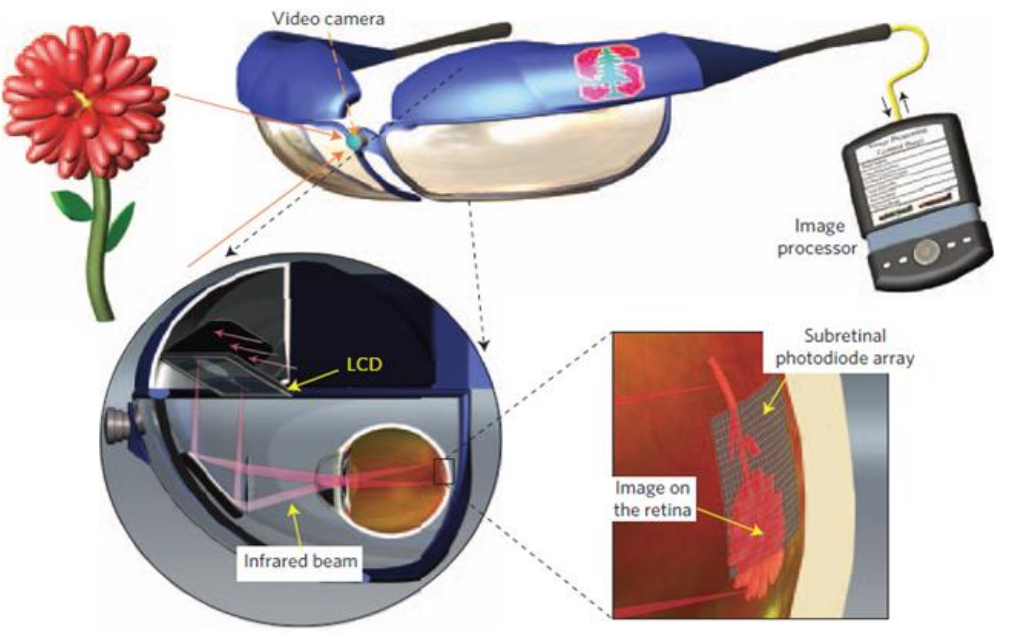

Figure 11. System design for a patient. The glasses contain a camera and projection system connected to a small pocket computer that processes the image before generating an IR image to project on the prosthesis. (Mathieson et al., 2012)

light in the NIR range as compared to visible light of the same intensity. The design of the system for patient use includes a pair of glasses mounted with a camera and projection system (Figure 11). The camera captures the visual scene and sends this information to a small pocket computer to process the image. The computer then sends a signal to the projector system contained within the glasses. With software to track eye movements, the projector generates a NIR image and transmits NIR light directly onto the prosthesis. In addition, this could allow any residual visual processing in the patient's retina to still be utilized with visible light either from ambient light (possibly using a dichroic mirror) or visual light projected from the system. Theoretically, the two signals should add together to enhance visual perception.

\section{Experimental Goal}

The goal of these experiments was to characterize the retina-prosthesis interface by showing the devices provide effective, repeatable and safe stimulation of the retina that results in visual signals that are transmitted to a 
visual structure of the brain. The two prosthesis designs were compared to determine if the bPVA presents a significant improvement over the mPVA design in terms of spatial activation and threshold. In my experiments, electrophysiological recordings were used to compare the MPVA and the bPVA retinal prosthetic designs in terms of their ability to drive high resolution, low threshold responses. As in DeMarco et al., (2007), my experiments used an intact preparation to record responses in the SC as an indicator of the prosthesis/retina interface, while stimulating the prosthetic device in situ.

\section{Materials and Methods}

\section{Animals}

All experiments were carried out with the approval of the Institutuional Animal Care and Use Committee (IACUC) of the University of Louisville. The Tg S334ter-3 rat was used for all experiments described in this chapter (Chapter II). Homozygous Tg S334ter-3 rats were acquired from Dr. Matthew LaVail (University of California, San Francisco). Homozygous Tg S334ter-3 rats were generated on the albino Sprague-Dawley strain of rat. Because albinism affects retinal function, homozygous $\mathrm{Tg}$ S334ter-3 rats were bred with Long Evans pigmented rats to create hemizygous Tg S334ter-3 pigmented rats (one copy of the transgene and two normal rhodopsin genes). Hemizygous Tg S334ter-3 rats were the experimental animals and Long Evans rats served as normal controls (Charles River Labs). 


\section{Implant Surgery}

Experimental animals are first anesthetized with an initial cocktail of ketamine $(85 \mathrm{mg} / \mathrm{kg})$ and xylazine $(7.5 \mathrm{mg} / \mathrm{kg})$. Once anesthetized, the pupil is dilated with tropicamide and phenylephrine. A suture is placed in the upper eyelid and traction applied to expose the superior portion of the eye. The eye is treated with proparacaine as a topical anesthetic and a second suture is placed in the superior conjunctiva near the limbus and the eye is rotated forward to expose the superior conjunctiva. The conjunctiva is then dissected away to expose the sclera. A 19-gauge vitrectomy knife is used to make a $1 \mathrm{~mm}$ incision through the sclera and the retina. Ten-minutes after a retinotomy, the retina will naturally detach from the RPE at the retinotomy site, which creates a space to implant the prosthesis. The prosthetic device is then manipulated carefully into the retinotomy site and placement is verified with a fundus lens. Closure of the retinotomy site has not been necessary because adverse effects (infection, vitreous exudation, etc.) at the retinotomy site have not been oberved.

Two to four weeks after implantation, the surgical outcome was assessed with fundus imaging, optical coherence tomography (OCT) and fluorescein angiography (Figure 13). From the fundus image I determined the location of the prosthesis in relation to the optic nerve. OCT images were used to determine the general health of the retina. This technique generates perpendicular images through the retina can help identify problems such as edema, gliosis, or other trauma near the prosthetic device. The retinal vasculature is also an important indicator of a successful surgery and the health of the retina. Fluorescein was 
used to illuminate vessels within the eye, after the dye is injected in the intraperitoneal cavity. Once in the vascular system, the dye is transported to the retina and illuminates the retinal vasculature for assessment. A successful surgery will show vasculature over the entire prosthesis as shown in Figure 12. If the prosthesis was not subretinal or there were conspicuous complications within the retina, the animal was not used for experimental purposes.
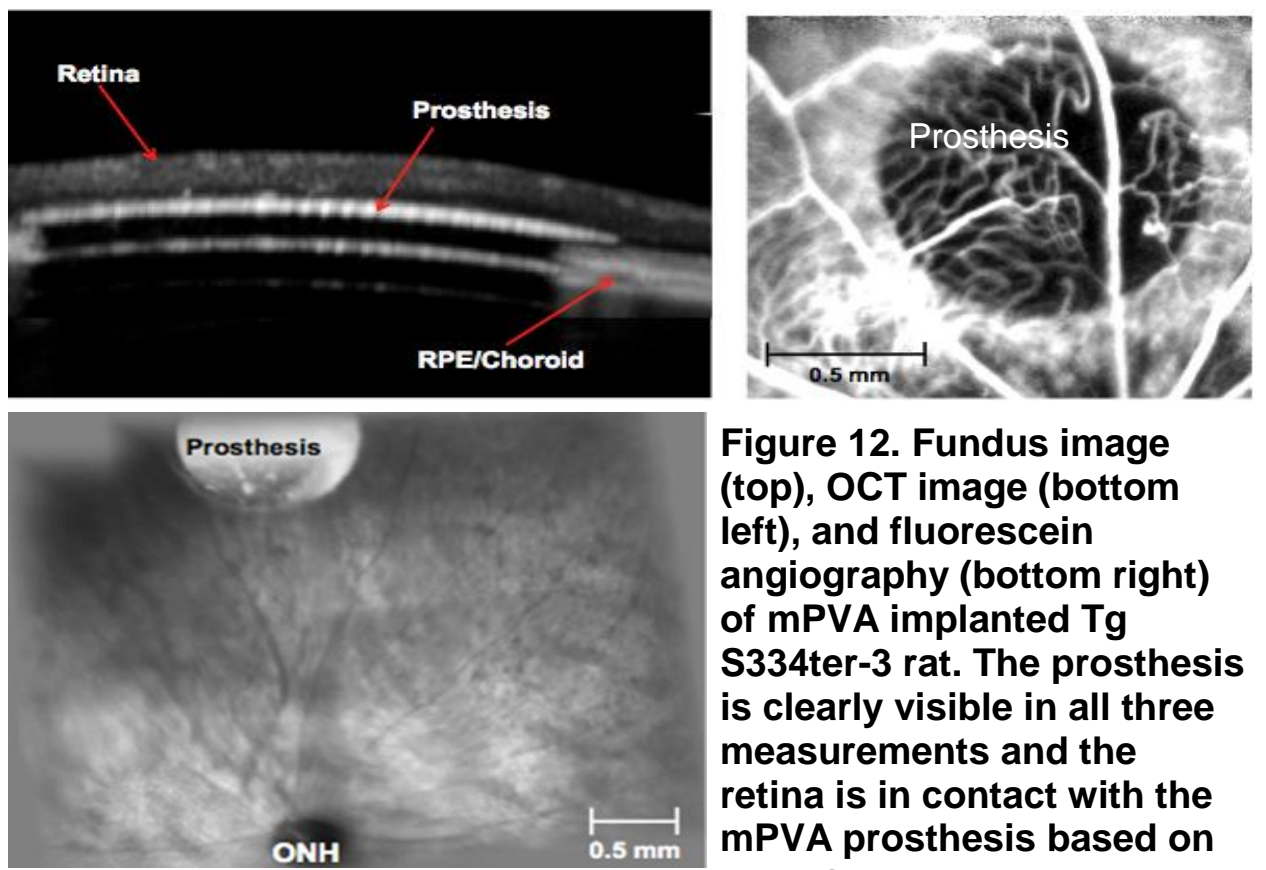

Figure 12. Fundus image (top), OCT image (bottom left), and fluorescein angiography (bottom right) of mPVA implanted Tg S334ter-3 rat. The prosthesis is clearly visible in all three measurements and the retina is in contact with the mPVA prosthesis based on the OCT image.

\section{Experimental Surgery}

Animals were anesthetized with the same cocktail used for the implant surgery. A diluted anesthesia cocktail (1:1 in ringer's solution) was administered throughout the duration of the experiment via a catheter inserted into the femoral vein and an infusion pump. Once the catheter was in place, the animal was immobilized in a stereotaxic. The skin on the dorsal surface of the skull was cut down the midline and retracted to expose the skull surface. A micro-drill was then 
used to create a $\sim 1.5 \times 1.5 \mathrm{~mm}$ square craniotomy in the skull. The cortex was then aspirated to expose the SC surface. Custom made lacquer coated tungsten electrodes $(0.25-2 \mathrm{M} \Omega)$ were used to record extra-cellular activity in either the SC or optic nerve.

\section{Electrophysiology}

To record and assess prosthesis-evoked activity in the SC, a similar design to DeMarco et al. (2007) was employed but incorporated the use of targeted stimulation of the prosthetic devices. A modified PASCAL photocoagulation laser (Topcon Medical Laser Systems) was used to direct a laser directly to the prosthetic device. Normally, the PASCAL system is designed to heat tissue within the retina to destroy portions of the retina or vasculature. This can be used to treat retinal detachment, diabetic retinopathy, and the wet form of age-related macular degeneration. In our application, the system provides an infrared laser (905 $\mathrm{nm}$ ) to stimulate the prosthetic device or a green laser (535 $\mathrm{nm}$ ) for visual stimulation, alignment and to precisely target the prosthesis. The laser power has been reduced from power levels that damage the retina $\left(\sim 300 \mathrm{~mW} / \mathrm{mm}^{2}\right.$ for a

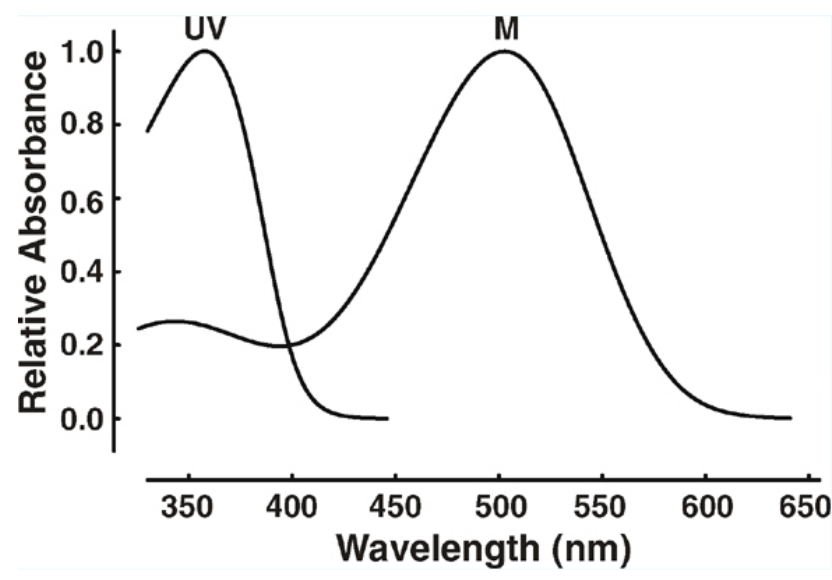

Figure 13. Spectral sensitivity of the UV and $M$ cones in rats. Jacobs et al., 2001. 
single pulse) to innocuous levels for the retina $\left(\sim 2.4\right.$ to $\left.19.1 \mathrm{~mW} / \mathrm{mm}^{2}\right)$. A custom LabView program (National Instruments Corporation) drives the laser and modulates the pulse width (duration) and intensity. The laser spot size can be adjusted to smaller or larger through the optics within the laser system itself or by changing the fiber optic cable that transmits the laser beam. For both prosthetic devices, the subretinal approach for implantation is utilized. In addition to the advantages of NIR light to stimulate silicon, I can be certain that any evoked activity with NIR light is generated through the prosthesis. Like mice, rats are dichromats with a medium/long (M) cone and a UV-cone (or S cone), with peak absorbance at 359 and $509 \mathrm{~nm}$ for the UV-cone and M-cone respectively (Figure 13). The M-cone spectral sensitivity is far below the $905 \mathrm{~nm}$ NIR light used for this study. Therefore, stimulation with $905 \mathrm{~nm}$ NIR light will not stimulate either cone type.

Multi-cellular responses were recorded from the SC using a single, lacquer-insulated tungsten electrode. To map the spatial extent and retinotopy of the prosthesis-evoked responses, the entire exposed dorsal surface of the SC was recorded by stepping the electrode in 200 micron steps across 54 distinct sites within the SC. The electrode depth was typically 150-400 microns, consistent with the extent of the superficial layers of the SC. Trials consisted of 20-25 stimulus presentations, with successful stimulation being defined as clear responses in at least $50 \%$ of the trials. 


\section{Data Analysis}

All data were collected and processed in Spike2 (Cambridge Electronic Designs, Cambridge, England). For statistical analyses, Prism 5.04 (Graphpad Software, La Jolla, CA) was used. Normally distributed data were analyzed with parametric statistics and a $p$-value $<0.05$ was considered significant. Unless noted, the data were normally distributed.

\section{Results}

Direct stimulation of either type of prosthetic device yields short latency responses that are transmitted to the $S C$ via retinal synaptic input from $B C s$ to RGCs

The Tg S334ter-3 rat was used to assess the interface between both types of PVA and the retina. Devices were implanted at either 35 or 75 days of age and evaluation times post-surgery were grouped into 50-90 and 120-150 days. An example of a prosthesis evoked response to direct IR stimulation is shown in Figure 14. Direct IR stimulation of the PVA produces prosthesis-evoked responses in $80 \%(n=21 / 25)$ of mPVA implanted and in $95 \%(n=20 / 21)$ of bPVA implanted rats that were implanted successfully. This high success rate represents an improvement over previous work with the same device in the RCS RP model, where 64\% ( $n=9 / 14)$ showed mPVA prosthesis-evoked responses. I believe that this is due to the use of the new PASCAL stimulation system as it combines the visualization of the device with direct presentation of the stimulus onto the prosthesis (both visible and IR). In contrast, a full field IR stimulus was used by Demarco et al. (2007). 


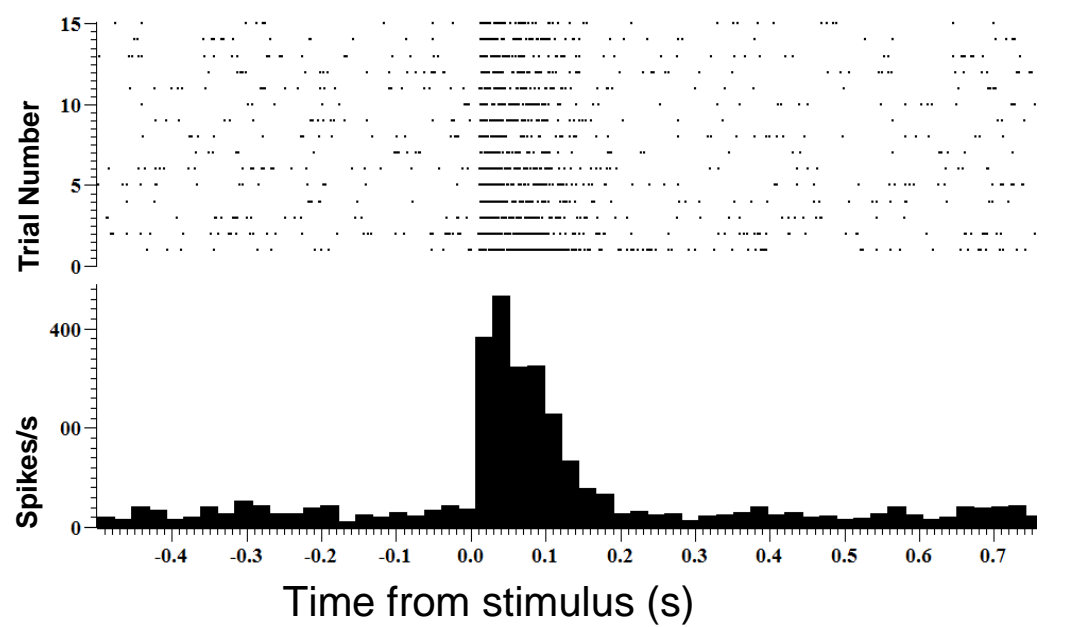

Figure 14. Raster plots and post-stimulus time histogram of prosthesis-evoked responses. The stimulus is pulsed at time 0 with 0.5 seconds shown before and $0.75 \mathrm{~s}$ shown after the pulse.

In addition to its selectivity for IR stimuli, both mPVA and bPVA

prosthesis-evoked responses can be distinguished from visually-evoked responses (with the same duration) by their significantly shorter time to peak (Figure 15A; 53 and $43 \mathrm{~ms}$ vs. $176 \mathrm{~ms}$ ). The responses evoked by the prosthetic devices also had a significantly shorter duration than the visible stimulus of the same pulse duration. The bPVA-evoked responses were slower to peak and had a longer duration compared to mPVA-evoked responses (Figure 15B). A shorter onset latency for PVA evoked responses is consistent with the idea that responses are transferred through one less synapse (photoreceptor to BC) compared to visually evoked responses. The integration time of light adapted photoreceptors is $40 \mathrm{~ms}$ (McNaughton, 1990), partially accounting for the difference in time to peak. When added with the transmission time of approximately $100 \mathrm{~ms}$ from $\mathrm{BC}$ transmission to $\mathrm{RGC}$ response (Baylor and Fettiplace, 1977), these estimates suggest that the devices stimulate BCs directly. To address this question, synaptic blockers were used to prevent synaptic signaling within the retina. If the PVA-evoked responses are transmitted 
through the retinal circuitry, it would be expected that synaptic blockers would eliminate PVA-evoked responses. If responses are present, it would indicate that the PVA is acting directly on RGCs.

A.

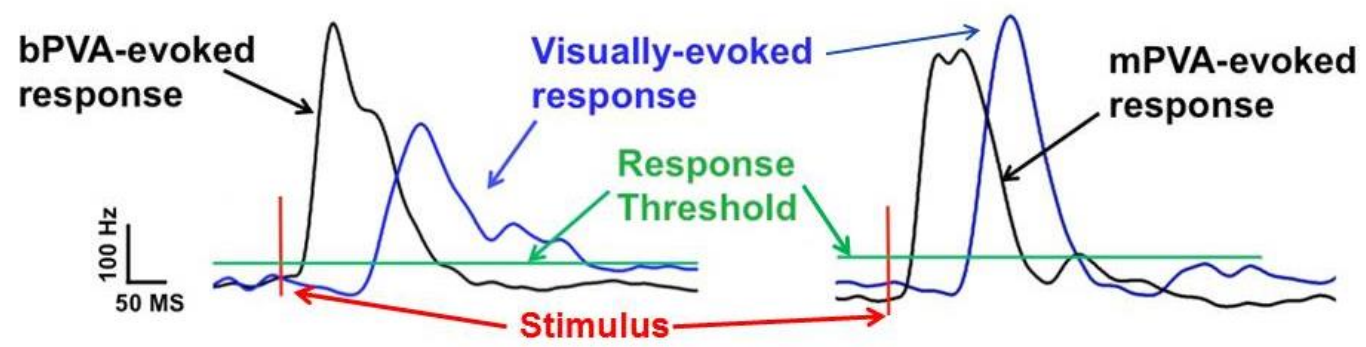

B.
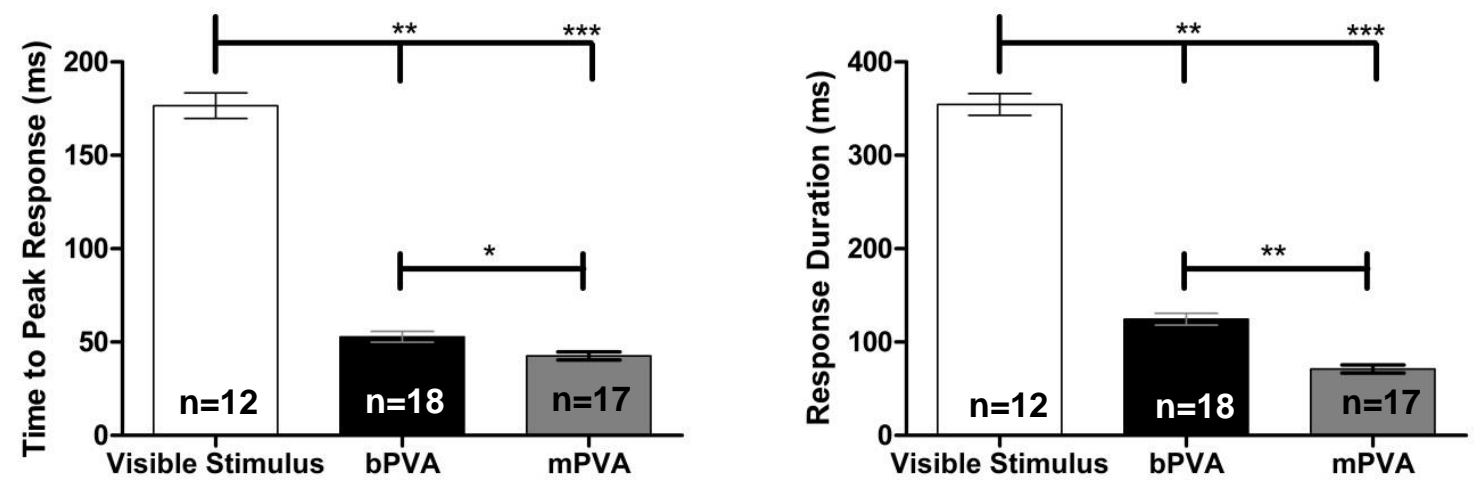

Figure 15. PVA-evoked responses respond with a shorter latency and duration than visually-evoked responses in the same animals. A - A comparison of the implant evoked responses (black trace) with the visually evoked response (blue trace) from a bPVA (left) and an mPVA (right). In each case the stimulus duration was $4 \mathrm{~ms}$. $B$ - A quantitative comparison of two aspects of the evoked response shows that implant evoked responses are significantly faster to peak and shorter duration compared to visually evoked response. ${ }^{*} \mathrm{p}<0.05,{ }^{* *} \mathrm{p}<0.01,{ }^{* * *} \mathrm{p}<0.001$

In four rats, an SC location with a robust prosthetic-evoked response was selected for the synaptic blocker injection. While recording the spontaneous activity, $2.5 \mu \mathrm{l}$ of saline was injected into the vitreous chamber. Injections of saline did not reduce the spontaneous activity or eliminate implant evoked responses in the SC (Figure 16A, B, D). Five minutes after the saline injection, an injection of $2.5 \mu$ containing a cocktail of synaptic blockers (10 $\mu \mathrm{M}$ strychnine, 
$10 \mu \mathrm{M}$ picrotoxin, $50 \mu \mathrm{M}$ TPMPA, $50 \mu \mathrm{M}$ D-AP5, and $10 \mu \mathrm{M}$ CNQX) was made. When the blockers were present synaptic activity was largely eliminated and bPVA-evoked responses were no longer elicited (Figure 16C, D). The effect of the synaptic blockers was verified with a steady decline in spontaneous activity, which was usually evident within 3-5 min after injection. These results indicate that the prosthetic directly stimulates cells in the INL and not RGCs directly.

A

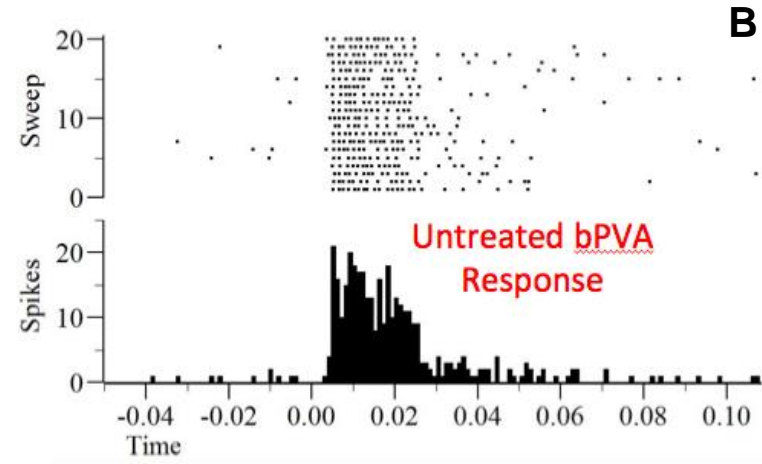

C

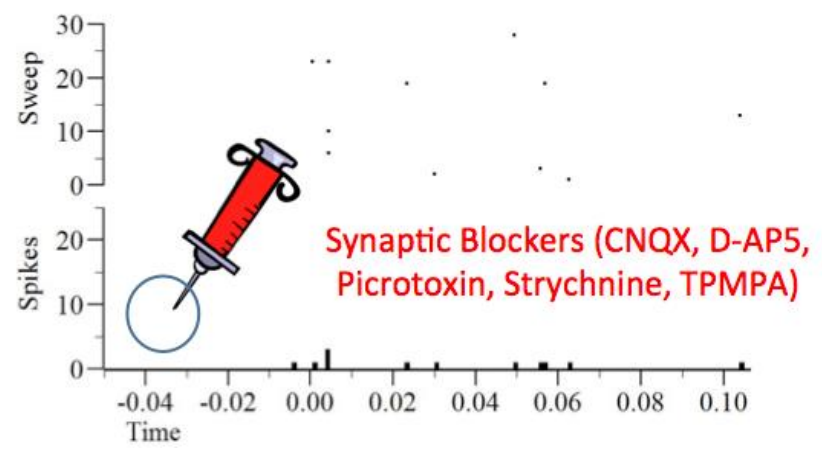

B $\left.\begin{array}{c}30 \\ 20- \\ 10- \\ 0\end{array}\right]$

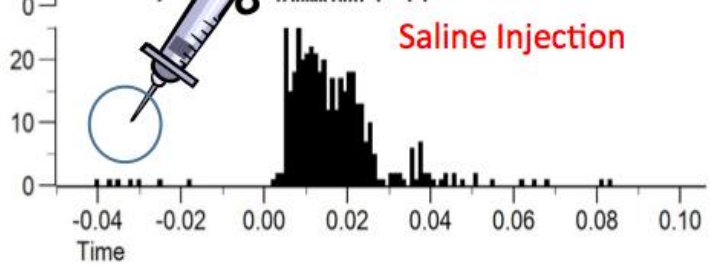

D

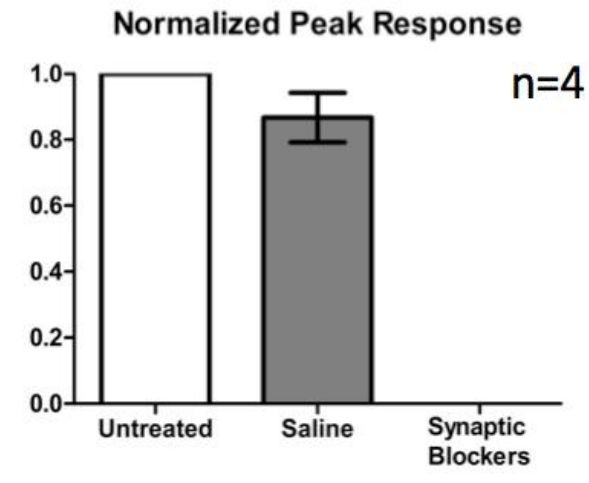

Figure 16. The bPVA response in the SC requires synaptic transmission of the signal through the inner retinal circuit. A. Representative averaged response and raster plot of bPVA evoked response. B. Injection of $2.5 \mu$ of saline did not significantly alter the bPVA-evoked response or spontaneous activity in the same animal. C. Injection of the synaptic blocker cocktail (2.5 $\mu$ l total volume) eliminated all bPVA-evoked responses. D. Average normalized peak response across four animals.

Given that the responses are network dependent, the additional reduction in time to peak of the IR-evoked responses is likely due to direct depolarization of the BC, thereby bypassing the PR-BC signalling cascade. The light-evoked time to 
peak response in WT rat BCs is $\sim 80 \mathrm{~ms}$ for ON BCs, $\sim 100 \mathrm{~ms}$ for OFF BCs, and $\sim 175 \mathrm{~ms}$ in rod BCs (Euler and Masland, 2000). In normal signalling, glutamate binding opens iGluRs directly in OFF BCs while in ON BCs, a reduction in glutamate binding inhibits a G-protein coupled cascade that normally closes the TRPM1 channel. These signaling processes act to depolarize BCs and open voltage gated calcium channels at the $\mathrm{BC}$ axon terminals. The increase in intracellular calcium causes the release of vesicles from the axon terminal. These channel gated and G-protein related processes in the BC dendrites are likely to be bypassed when stimulated by a PVA. The latency, therefore, would be limited to the time for calcium entry into the $\mathrm{BC}$ terminals, vessicular release, neurotransmitter diffusion across the BC to RGC synapse, and depolarization of the RGC.

In addition, Sekirnjak et al. (2008) reported direct activation of RGCs in monkey retina resulted in sub-millisecond latency to response. In no experiment did I record responses in the SC with a latency that was less than 35 milliseconds. Taken together, the above data strongly support my hypothesis that the bPVA devices stimulate the retinal network rather than RGCs directly.

Direct stimulation of the prosthetic device yields responses in a location generally matched to the position of the implant in the retina.

Since the SC forms a retinotopic map, the IR-evoked responses should correlate with the location of the device in the eye. Without a predictable relationship between the location of the prosthetic and the area of the retina stimulated, spatial information would not be reliably relayed to the patient. To 
address whether or not the predicted location of stimulation matched the observed location of stimulation, fundus images for each implanted rat were taken, which included the optic nerve head $(\mathrm{ONH})$ and the device. The location of the device relative to the $\mathrm{ONH}$ along the vertical and horizontal meridians were computed and compared to the sites of evoked activity on the SC retinotopic map. Figure 17 shows representative images of the fundus of two rats implanted with an mPVA (top) and a bPVA (bottom). The corresponding PVA evoked responses are plotted on the right as a heat map of the dorsal surface of the SC. Responsive areas appear as colors, where red represents the strongest excitatory responses and blue the weakest responses. Black areas indicate locations in the SC where prosthetic evoked responses were not elicited. I compared the location of PVA evoked responses recorded in the SC to their expected location based on implant placement in the eye. The fundus image was used to compute an estimate of the expected location of PVA influence in the SC. To do this, I first measure the distance to the center of the device from the center of the optic nerve head along the vertical (superiorinferior) and horizontal (temporal-nasal) axes (Figure 18A). These two distances are converted to visual degrees where $59 \mu \mathrm{m}$ subtends $1^{\circ}$ of visual angle in the rat eye (Siminoff et al., 1966). These estimates are then compared to the location of the peak PVA-evoked responses in the SC using an image adapted from Siminoff et al. (1966, Figure 18B). Each line on the SC map in Figure 18B represents 10 degrees and the optic nerve has a defined position. Using the fundus image estimates, the difference between the two locations was calculated 
along each axis. For reference, the bPVA device is $1.2 \times 0.8 \mathrm{~mm}$ which corresponds to $20 \times 13.5$ degrees of visual angle.
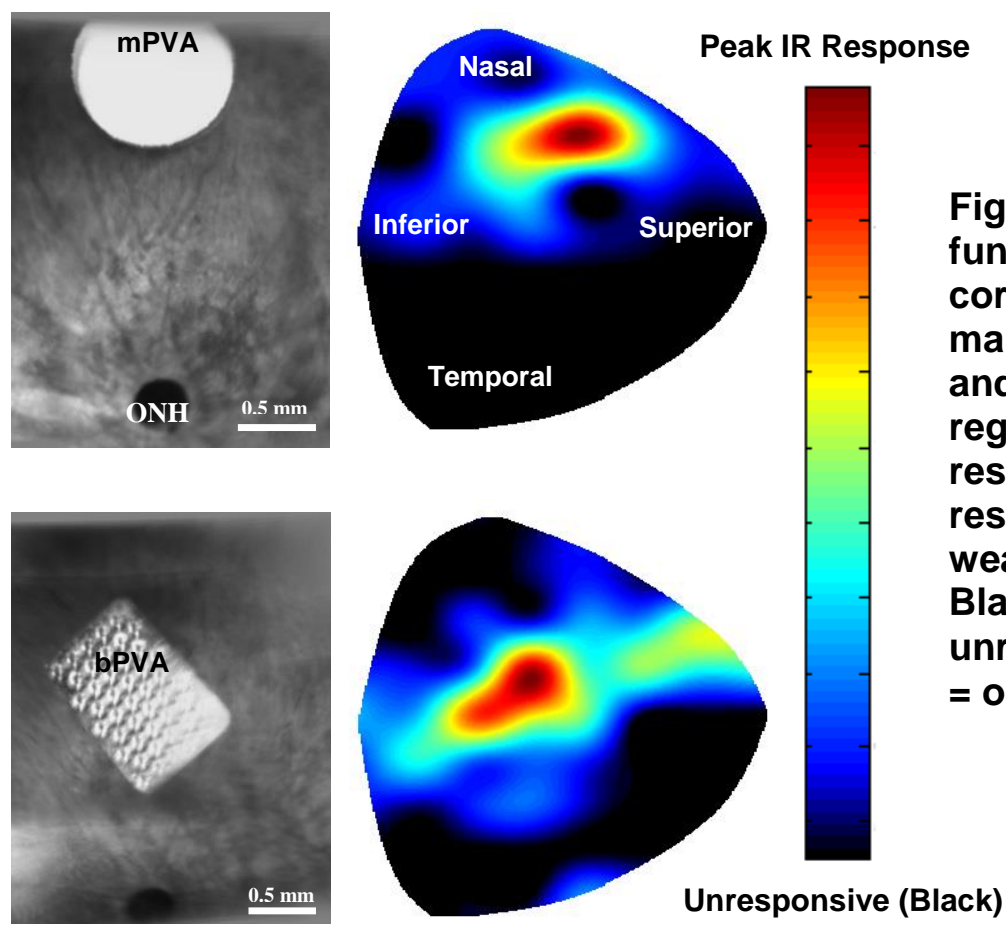

Figure 17. Representative fundus images and corresponding SC activity maps. The top fundus image and map show a localized region with prosthesis-evoked responses. Stronger responses are in red and weaker responses in blue. Black represents unresponsive locations. $\mathrm{ONH}$ = optic nerve head. 
A

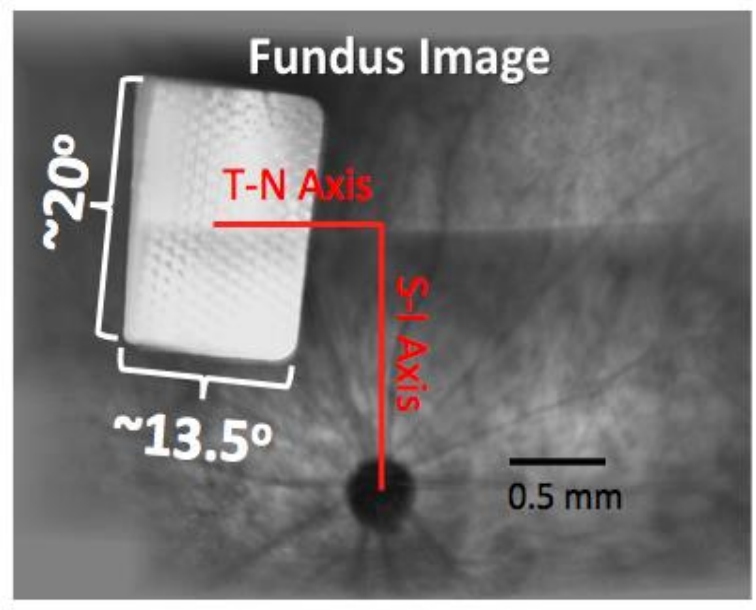

B

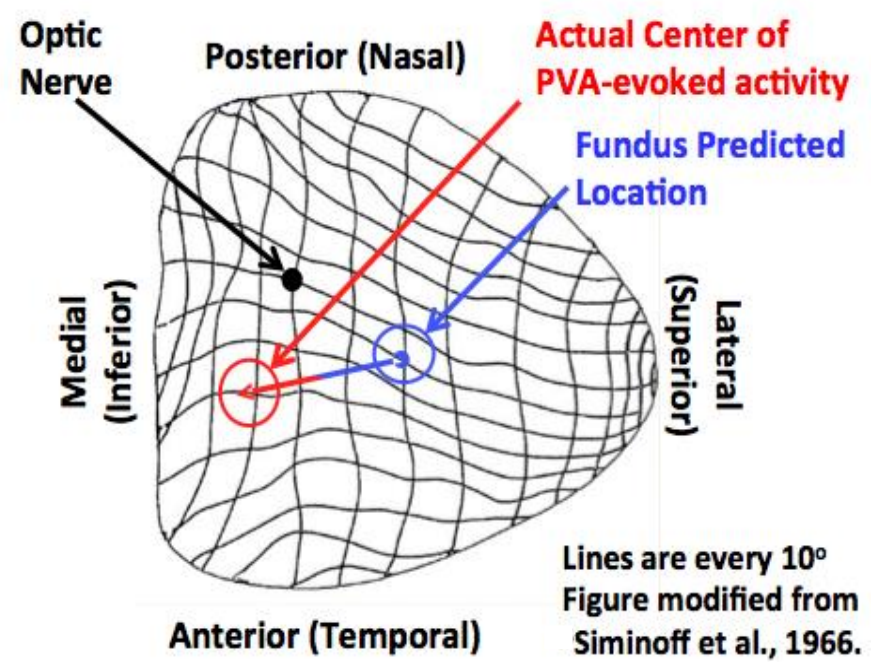

Figure 18. A. Fundus image showing the size of the implant in visual degrees and the distance from the optic nerve along both the S-I and T-N axis. B. For the animal shown in $A$, the deviation of the $\mathrm{SC}$ recorded activity from the predicted location is plotted on the dorsal surface of the SC. Each line on the SC map represents ten visual degrees.

Figure 19A plots the predicted location of the PVA as a function of the location of its actual evoked activity. Each arrow represents one animal and the length of the arrow represents the change between predicted location (blue dot) and the actual center of the recorded activity in the SC (red arrow head). The rat retina covers approximately 140 degrees along the T-N axis and 130 degress across the S-I axis (Siminoff et al 1966). Figure 19B plots a summary of the deviations from the predicted location for all rats. On this graph, a perfect match between predicted and actual is represented by the green circle at the origin $(0,0)$. Given the size of the retinal representation, the differences I observed are fairly restricted. However, there is a trend, in 22 out of 23 rats the predicted location is superior to the actual area of activity. Additionally, in 16 out of 23 rats the predicted location is nasal to the actual area of activity. Given the consistency of the direction of difference, it most likely indicates a consistent error in one of our 
estimates of location on the SC surface due to errors of electrode placement (discussed below) or differences in the visual map with the estimates of Siminoff et al., 1966. In addition, there is the possibility that the implant could have changed location in the time between imaging the fundus and electrophysiological assessment (2-4 weeks).

A.

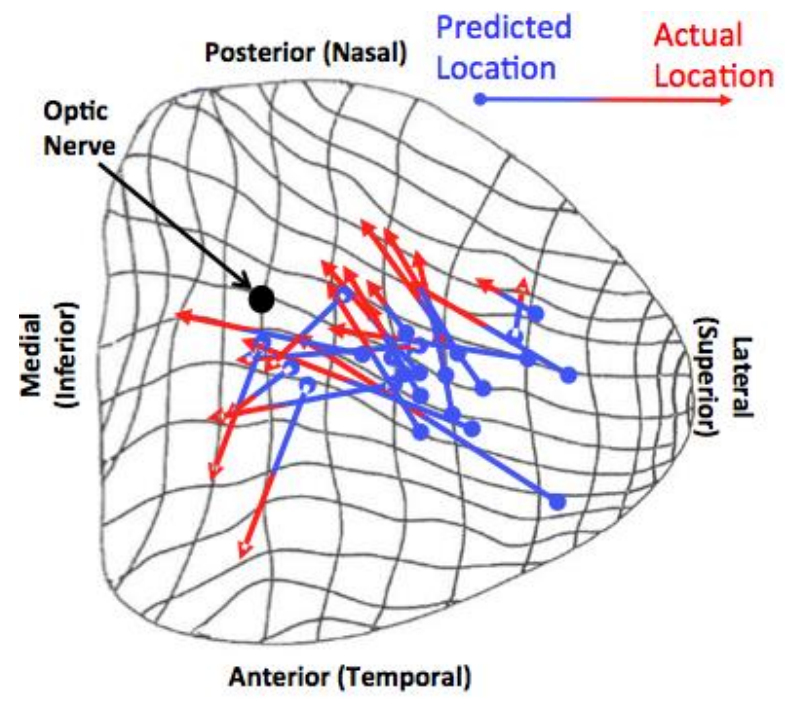

B.
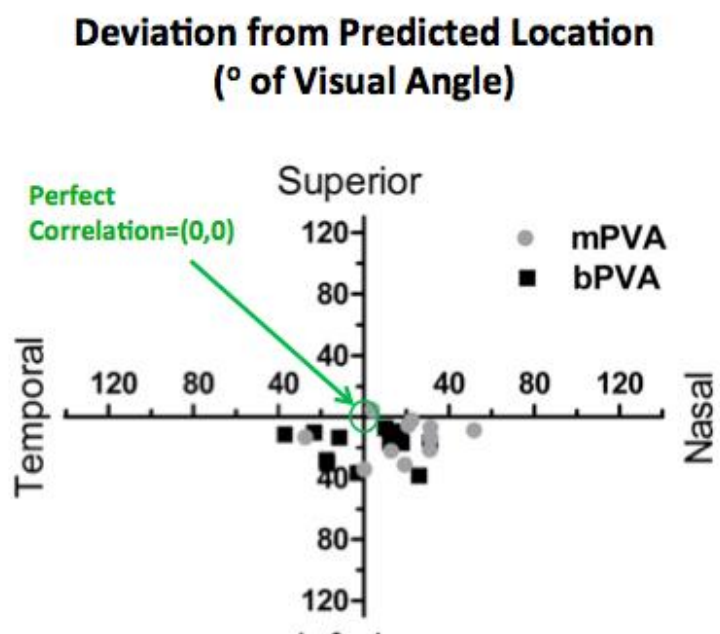

Inferior

Figure 19. A. SC representation of each animal's predicted vs. actual location of PVA influence. B. The deviation from the predicted location. Perfect correlation between the predicted and actual area of PVA influence in the SC would be at the origin $(0,0)$.

Measurement errors were introduced due to the way I estimated the location of the device based the fundus image and also due to the recording protocol. The device itself covers up to $20^{\circ}$, but the fundus measurement was taken to the center of the device. This introduces an error of up to $10^{\circ}$ due to the size of the device as the recorded activity was not necessarily from the center of the device. Current spread at the edges of the prosthesis also makes the device functionally slightly larger than its physical size by $\sim 200 \mu \mathrm{m}\left(3.5^{\circ}\right)$ at each edge (unpublished obervations by our collaborators). Our estimates of SC location 
may contribute as a result of anatomic variation. Blood vessels on the surface of the SC are used as a reference for placement of the first recording site, but there can be substantial anatomical variation from animal to animal. When all sources of error are considered, I compute a potential error of $30^{\circ}, 18$ out of 23 locations fall within this predicted error range. Data points outside of this margin of error may indicate an implant interface directly with RGCs and their axons crossing under the PVA. This would create large deviations from the predicted location. Alternatiely, devices may have physically shifted more in these animals over time. Because there were so few of these animals and the calculations were made after experiments, I did not test the idea that RGCs were directly stimulated in these animals.

Implantation age and duration have no significant effect on thresholds for mPVA evoked responses

The threshold for stimulation is a concern with implant devices, as too much current can cause damage to both the retina and the device. Further, there could be differences in the interface between the implant and the degenerating retina as photoreceptor death proceeds. To examine these questions, I compared thresholds in two groups of Tg S334ter-3 rats implanted at different stages of degeneration, $\sim$ P35 and $\sim P 75$. I also varied the time between implantation and evaluation in both implantation age groups: $120-150$ days post implantation. At P35 there are 3-5 rows of nuclei left in the ONL while P75 animals only have $1-2$ rows of nuclei remaining, responding exclusively to 
photopic stimuli. Figure 20 compares the threshold responses across implantation age (A) and duration (B). The data show that neither implantation age nor duration have a significant effect on threshold, consistent with data reported by Chan et al (2011).
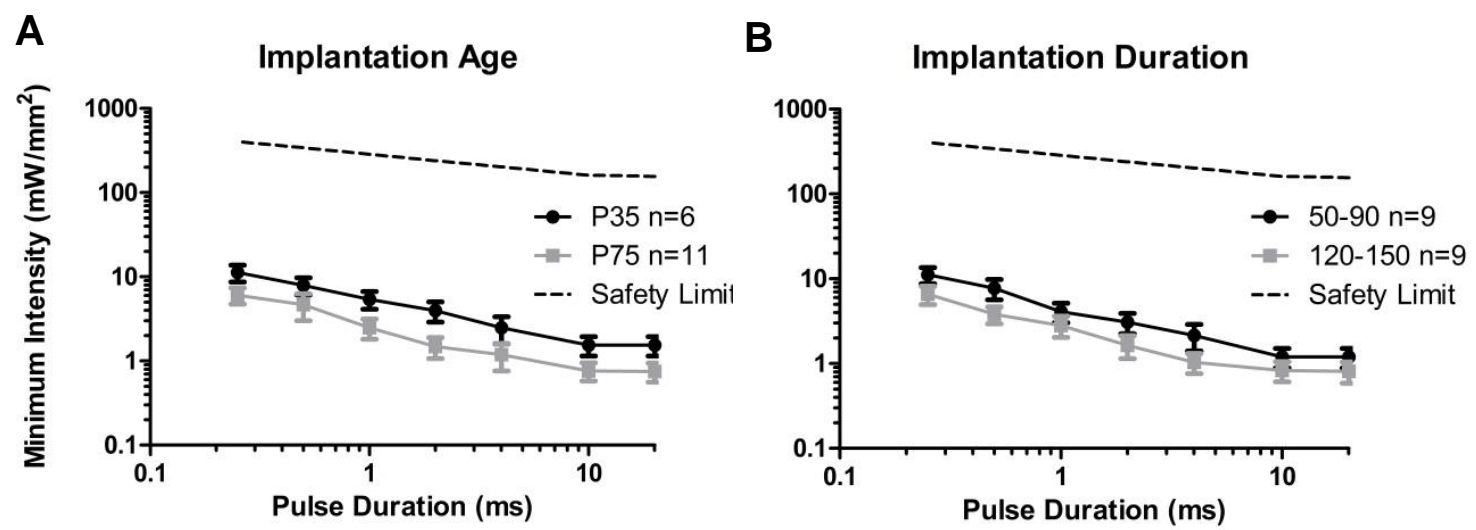

Figure 20. The figures plot the minimum intensity required to elicit a response at a particular pulse duration. A. Implantation age had no effect on thresholds for stimulation. B. Implantation duration also did not affect stimulation thresholds. Note all thresholds are at least $1.5 \mathrm{log}$ units below safety limits (dashed line) for the retina.

Threshold for stimulation is not different across pixel sizes in bPVA devices but bPVA devices have a significantly higher threshold than mPVA devices.

Theoretically, reducing pixel size and increasing density will increase visual spatial resolution. However, pixel size affects current density which can affect lateral current spread. To compare the effect of pixel size on threshold, three groups of animals were implanted with small $(70 \mu \mathrm{m})$, medium $(140 \mu \mathrm{m})$, or large $(280 \mu \mathrm{m})$ pixel bPVA devices. Figure 21 compares the threshold responses across pixel size within bPVA devices (A) and between mPVA and bPVA devices (B). The data show that pixel size did not significantly influence threshold for bPVA devices. Figure 21A shows the comparison across pixel size with all 
having similar thresholds. When compared to mPVA devices however, bPVAs have a consistently higher threshold. This is probably a function of their smaller current spread, which is more spatially limited than the bPVA device. The current generated by smaller electrodes will not spread as far, requiring either a closer interface between the electrode and the tissue to be stimulated or for the electrode to produce more current (Palanker et al., 2005). Thus, higher threshold in bPVAs is consistent with expectations. It is important to note that all thresholds are well below the defined safetly limit for these devices in the retina.
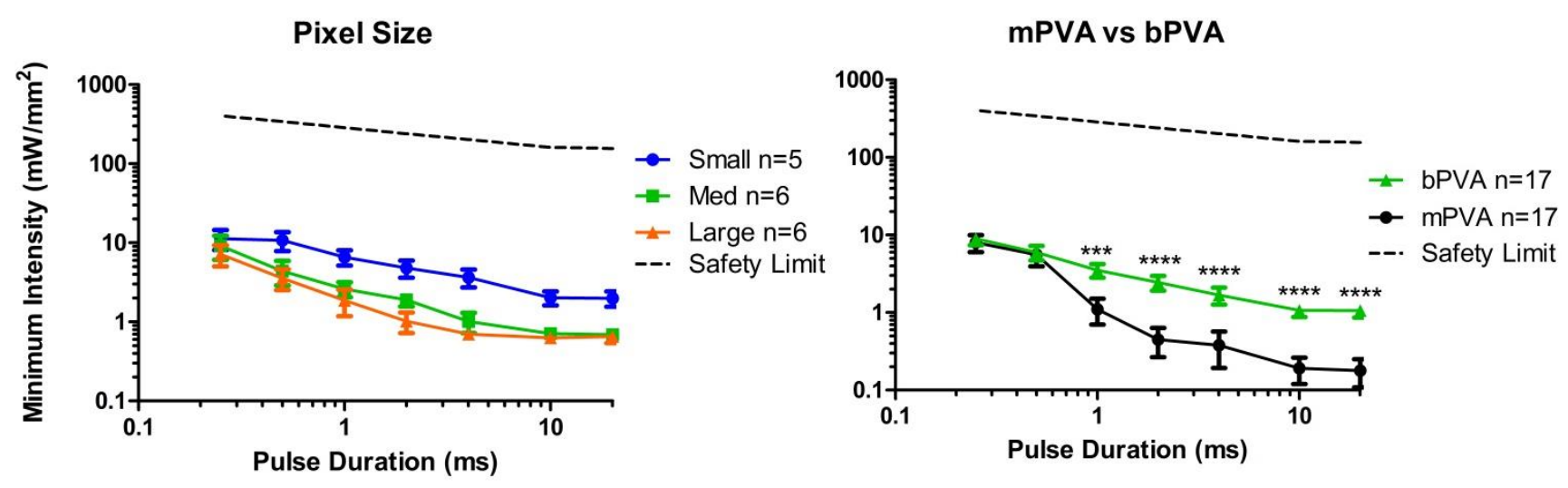

Figure 21. Device thresholds are well below the safety limits. A. The intensityduration plot comparing bPVA pixel sizes. Medium and large pixels were not significantly different from one another. B. Comparison between small bPVA, combined medium and large bPVA, and mPVA device thresholds. mPVA thresholds were similar to the medium/large bPVA devices but small bPVA devices had a significantly higher threshold than the mPVA device thresholds. (Kruskal-Wallis). ${ }^{\star \star *} \mathrm{P}<0.001,{ }^{* \star \star \star} \mathrm{p}<0.0001$

PVA-evoked responses have a linear range as a function of pulse duration

The interaction between the retina and a prosthetic device is certain to shape the perception for a patient. In the intact retina, the output of the photoreceptor is proportional to the intensity of the light within a given adaptation range. Similarly, it is ideal if the amount of current produced by the implant is 
proportional to the intensity of the IR stimulus. In this way, larger portions of the retina or cells with higher threshold may be recruited when the device is driven harder to increase the current output of the device. In addition, the response limits of the retina are important to quantify in order to keep the stimulus intensity as low as possible (to prevent damage to the tissue) while eliciting consistent responses.

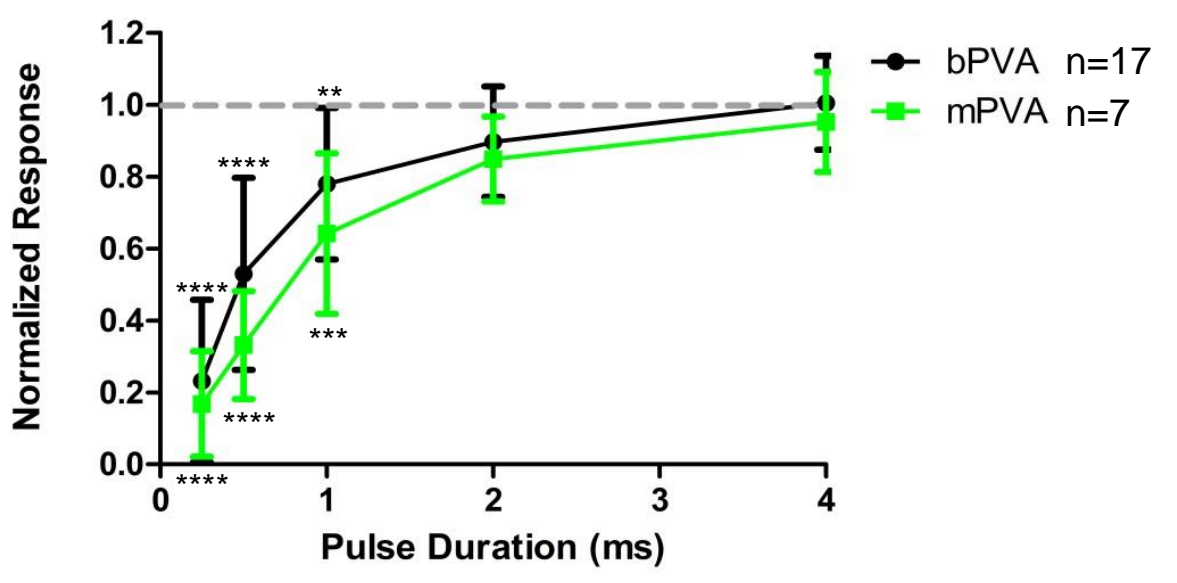

Figure 22. Normalized response as a function of pulse duration. Compared to the $10 \mathrm{~ms}$ response, both mPVA and bPVA devices show a linear range between 0.25 and $2 \mathrm{~ms}$. Beyond $2 \mathrm{~ms}$, the response does not significantly increase further. ${ }^{* \star} p<0.01,{ }^{* \star *} p<0.001,{ }^{* \star \star *} p<0.0001$

To determine if the peak SC response changes as a function of pulse duration, I recorded from the SC while modulating the pulse duration. Figure 22 plots these data and demonstrates a linear relationship between response and pulse duration between 0.25 and $2 \mathrm{~ms}$ with saturation at durations above $2 \mathrm{~ms}$. This relationship was the same for both bPBA and mPVA devices and there was no significant difference between device types. 
bPVA devices provide focal activation of the retina that is not seen with mPVA devices

At present there are devices that are being tested in human RP patients (Humayun et al., 2010). These implants have a limited number of electrodes with relatively wide spacing. While preliminary reports are promising, most patients can only use the device for simple pattern vision or luminance levels. However, the ultimate goal is to provide high spatial and temporal resolution vision to patients that have lost photoreceptors. To test the hypothesis that the bPVA design should activate the retina in a focal manner with increased spatial resolution over the mPVA, I measured the spatial resolution of mPVA $(n=12)$ and

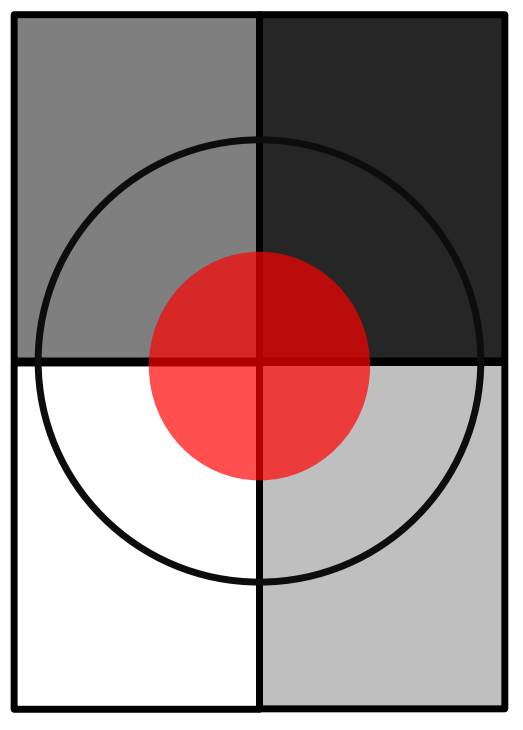

Figure 23. Diagram showing the five different stimulus locations for each device type. The larger circle represents an mPVA and the entire rectangle $a$ bPVA.
bPVA $(n=14)$ devices in implanted animals. An

SC site with a robust PVA-evoked response was selected and the response at this site was recorded as a small diameter spot stimulus (0.5 $\mathrm{mm}$ ) was systematically moved to one of five sites on the PVA. Figure 23 depicts the regions stimulated under this paradigm. Comparisons were made with the IR stimulus located in either the center or in one of four quadrants of the PVA. The comparison was made to determine if the response would increase or decrease depending on what region of the device was stimulated. Since the SC is topographically organized, 
stimulating the device in different regions should shift the area of response in the SC to match the region of the retina that is stimulated. In this way, recording at one SC site while moving the IR stimulus should reduce or increase the reponse recorded in the SC. If the response does not change, the entire device is stimulating regions of the retina that are larger than the stimulus. In this scenario, the entire device is functionally a single pixel, rendering the electrode array ineffective for any spatial resolution. Figure 24 plots representative
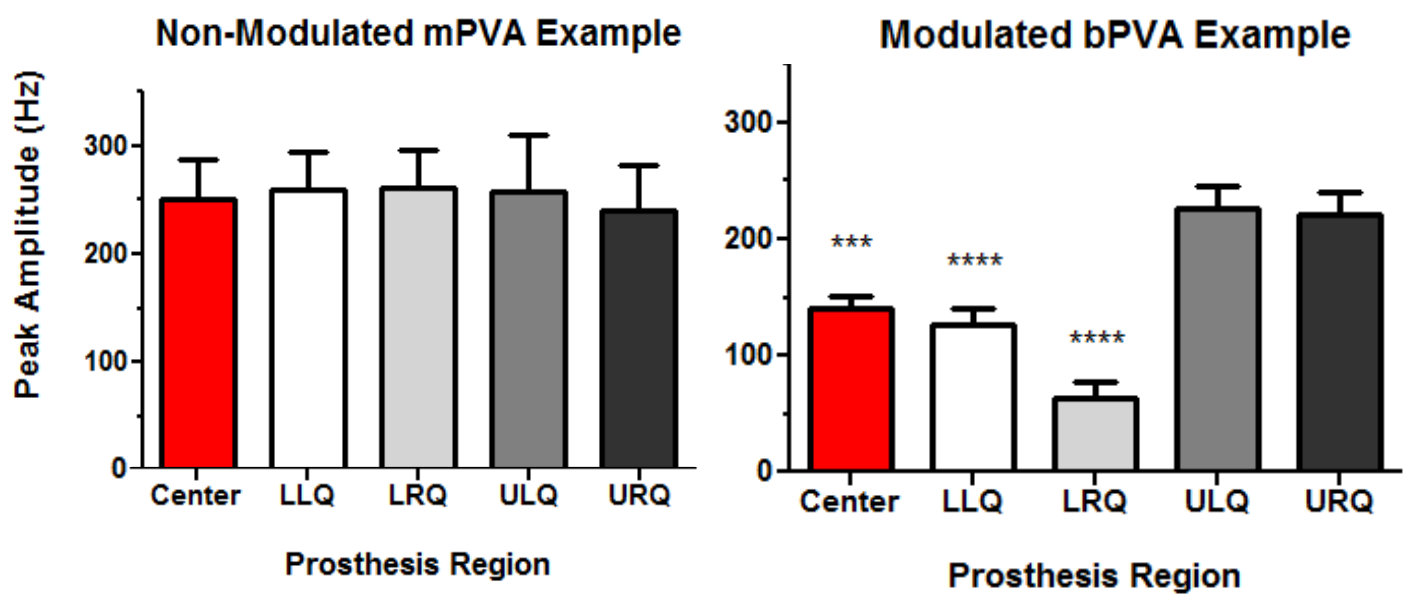

Figure 24. A. Histogram showing an example of the unmodulated response typical of mPVA devices. B. Example of a significantly modulated response from a bPVA implanted animal.

responses for an mPVA (A) and a bPVA (B) device. The responses evoked by spots across mPVA devices were similar in 14/17 animals (Figure 25), indicating little or no spatial resolution. In contrast, bPVA devices showed significant response change as a function of spot location in 22/23 animals, where the response at each region was compared to the largest response region across the device. I believe that the best explanation for response modulation in the $3 / 17$ mPVA is a poor interface between the prosthesis and the retina in one quadrant 
of the device. In these devices, the

quadrant with smaller responses may not

have been as closely juxtaposed to the

retina, resulitng in one region with a

significanly smaller response. These

results strongly support the idea that

current generated by bPVA devices is

able to flow locally because of the

individual return electrodes. This also

strongly supports the bPVA design as a

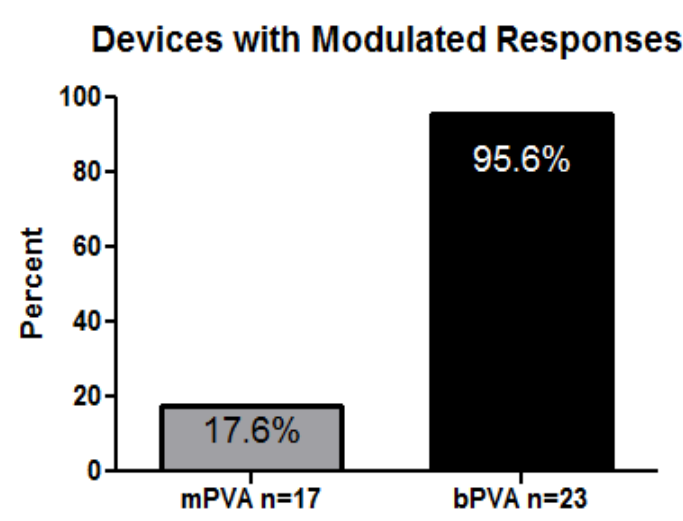

Figure 25. Overall, only 1 bPVA device did not show some modulation while only 3 of 17 mPVA devices showed any modulation when moving the stimulus location across the face of the PVA. significant improvement over older prosthetics.

\section{Discussion}

In comparing the mPVA and bPVA devices, my results demonstrate that the bPVA design is a significant improvement in prosthetic device design. The primary improvement is its superior spatial localization, while continuing to achieve low thresholds for activation that are far below established safety limits for the retina. The high integration success with the bPVA devices indicates a better interaction between the device and the retina than the older mPVA device. This is likely due to the targeted stimulus but may also result from the increased flexibility of the bPVA design. Jensen and Rizzo (2009) concluded that a biphasic pulse was the most consistent stimulus to use in the $r d 1$ mouse model of RP and my results with a higher success rate using bPVA devices support their 
conclusion. It was very evident that surgical implantation technique is paramount to a successful interface of the device with the retina. All animals without a clear interface between the device and the retina (OCT images) had no prosthesis evoked responses.

As manufacturing technology becomes more advanced, the size of the electrodes will undoubtedly decrese to theoretically provide increasingly focal stimulation of the retina. While it is important to increase the resolution of the devices and the perceived image, the safety of the retina must be considered as well. There was a trend for an increase in threshold in the smallest size pixel in the bPVA devices $(70 \mu \mathrm{m})$. In addition, there was a significant increase in thresholds for bPVA devices when compared to mPVA devices. While still far below safety limits, the challenge for new devices in the future will be a balance between the size of the electrodes and approaching safety limits. Any increase in risk to the patient or to the lifetime of the device must be carefully considered when attempting to improve the design of prosthetic devices.

It should be noted that other approaches also are being actively explored for the replacement of degenerated photoreceptors. For example, of great potential for restoration of vision for patients with degenerative disease is the transplantation of stem or other progenitor cells into the retina (Schmeer et al., 2012). The current state of this potential treatment is far behind retinal prosthetics. Early clinical trials using stem cells are currently under way though some trials ended prematurely due to safety concerns. In contrast, the FDA recently approved the first prosthetic device for use in humans. Second Sight 
Medical Products Inc. received approval for general use of their 60 electrode Argus II device in end stage RP patients in the US. Additionally, the state of the retina for stem cells or transplants may preclude patients with severely degenerated retinas. It has been shown that there are significant morphological and structural changes that take place in advanced stage human RP patients and animal models (Marc and Jones, 2003). In addition, the expression of mGluR6 receptors in the BCs has been shown to decline in multiple models of RP, including rd1 and 10 mice (Stasheff 2008, Puthussary et al., 2009; respectively) and RCS rat (Pu et al., 2006) while the expression of iGluR on OFF $\mathrm{BCs}$ is stable even after the loss of all visual signaling. If the retina can no longer respond to glutamate signaling, implantation of photoreceptors would be ineffective at these stages of degeneration the retina, even if synapses form between the stem cells and BCs. If this loss of glutamate receptors is evident in human RP patients as well, the best treatment option may be retinal prosthetic devices. In addition, embryonic stem cells require immune-suppressive therapy for the life of the patient, something that is not required with prosthetic devices. This is not a trivial matter as major health complications can arise when the immune system is suppressed.

The wide-spread use of retinal prosthetic devices is still in the beginning stages and many questions remain unanswered. Will retinal adaptation to electrical stimulation result in higher thresholds for activation over time? There is evidence for short-term adaptation on the order of seconds (Freeman and Fried, 2011) but long-term stability of thresholds in humans has not been definitively 
established. In many cases, the first response to PVA-stimulation did appear to be larger but this was not quantified. This analysis will be addressed in the future.

There is debate about what the most physiologically relevant stimulus is to the retina. It has been shown that rabbit RGCs can follow pulse trains up to 600 $\mathrm{Hz}$ when stimulated directly using tungsten electrodes (Cai et al., 2011) but normal human frequency limits are in the range of $45 \mathrm{~Hz}$. Does the stimulus have to transmit signals that are close to what is processed normally or will plasticity within the visual system enable it to adapt to this new information and interpret it as normal (or potentially supernormal) perceptions? Can a stimulation configuration be found to differentially stimulate specific cell sub-types to generate both ON and OFF signals? BCs are the point at which the ON and OFF pathways diverge, so stimulating all BC types at the same time may result in conflicting signals. On the other hand, there is evidence in the literature that patients with complete congenital stationary night blindness, where the retina lacks signaling through the ON pathway, have relatively normal day vision (Bijveld et al., 2013). Therefore, it is possible that the device does not require dual pathway signaling to provide normal vision.

Regardless of the answers to these questions, there is no doubt that retinal prosthetic devices integrate with the degenerate retina and generate signals that can be detected outside of the retina and can be used to represent some properties of the visual environment, in patients with photoreceptor degeneration. While the current visual acuity achieved with prosthetic devices in my studies as well as in work with human patients may be low (20/560 Zrenner et 
al., 2012 and 20/1260 with Argus II system, Humayun et al., 2012), this can still represent a life changing treatment. Many patients involved in clinical trials were late stage patients with little more than light or dark perception if any perception at all. The potential of these devices to provide independence for a profoundly blind patient cannot be understated. To have form, contrast, and/or depth perception again may allow them to navigate a room without bumping into objects, cross the street safely, or cook a meal easily; all things those with normal vision take for granted. 


\section{CHAPTER III}

\section{FUNCTIONAL ASSESSMENT OF TWO MODELS OF RETINITIS PIGMENTOSA INDICATE A PREFERENTIAL LOSS OF THE ON PATHWAY DURING THE DEGENERATIVE PROCESS}

\section{Introduction}

In many cases, patients with degenerative diseases of the retina do not present with symptoms until very late in the progression of the disease. Often the loss of vision is what drives patients to see a physician. Unfortunately, during the degenerative process within the photoreceptor layer, the rest of the retina is not static. Morphologically, there are numerous reports that show anatomical reorganization, but changes in function at the level of the RGCs have not been well studied. To better understand what treatments may be most effective, a description of these changes is needed. If early functional changes are found, this could facilitate early therapeutic intervention and may lead to new early screening procedures, all of which may lead to a better prognosis for patients with degenerative diseases. 


\section{Reactive Changes During Degeneration}

Morphologic changes that occur during the degenerative process have

been described previously. In rat and mouse models of RP, a combination of glial migration, vascular and RPE invasion, receptor expression changes, aberrant connections, and other changes have been described (Marc and Jones, 2003, Cuenca et al., 2004). Changes within the INL include neurite sprouting in horizontal cells, retraction of BC dendrites, and decreased mGluR6 staining (Strettoi et al., 2002). Aberrant connections form between rod BCs and cones in late stages of degeneration though it is not known if the synapses are functional (Marc and Jones, 2003, Cuenca et al., 2004).

In addition to structural changes, some morphological and functional changes have been reported. In the rd1 mouse, mGluR6 receptor expression on the $\mathrm{BC}$ dendritic tips is lost early in the degenerative process, while iGluR receptors (the OFF pathway) have been reported to persist and respond in vitro to AMPA/Kainate (iGluR agonists) puffs long after photoreceptors are lost (Stasheff, 2008). Functionally, the loss of RGCs with ON responses and the retention of RGCs with OFF responses have also been reported in the rd10 mouse (Puthessary et al., 2009, Stasheff et al., 2011) and the RCS rat (Pu et al 2006). The question remains, however, if this is something inherent to the degenerative process in general or if it is a result of a subset of specific mutations. The three examples listed above represent a mutation in rod photoreceptors (Pde6 gene) for the rd1 and rd10 mice and a mutation in the RPE (Mertk gene) for the RCS rat, suggesting this may be common to RP. However, 
Sekirnjak et al. (2011) reported that there was no differential loss of ON vs. OFF responses in the $\mathrm{Tg} \mathrm{P} 23 \mathrm{H}-1$ rat. They did find, however, that in the spontaneous firing rate of their OFF RGCs was elevated, while ON RGC firing rate declined. In both types of RGCs, receptive field size reduced with age, indicating a reduction in excitatory input. Importantly though, all RGCs were responsive to direct electrical stimulation even after light stimuli could no longer evoke a response (Sekirnjak et al 2009).

\section{Increased Spontaneous Activity and Rhythmic Bursting}

Many models of retinal dysfunction exhibit characteristic bursting in their spontaneous activity that is rhythmic. In the rd1 mouse, bursts of activity at $\sim 10$ $\mathrm{Hz}$ were found in both ON and OFF RGCs, but the morphology and dendritic stratification of these RGCs remained unaltered (Margolis et al 2008). This characteristic bursting activity has been described in other retinal mutants as well, including the nob mouse (Demas et al., 2006), Tg P23H-1 rat (Sekirnjak et al., 2009), and RCS rat (Sauve et al., 2001) among others. This suggests there are common functional changes within the retina when normal signaling pathways to RGCs are lost. If a common mechanism exists for changes in spontaneous activity, it is possible that there are other common functional changes across retinal degeneration models as well. 


\section{Experimental Goal}

The goal of these experiments was to describe some of the functional changes that are associated with the degenerative process. Responses were recorded from the SC in Tg rats and compared to age matched WT animals. Most previous research has focused on morphological changes within the retina but the functional changes have been explored to a lesser extent. To replace lost photoreceptors, either functionally or with cellular transplants, the remaining retina must be able to transmit signals. A description of functional changes will help guide future research and help determine the limits of proposed treatments. An in vivo preparation was used to determine what signals are transmitted to the SC from the retina as photoreceptors are lost.

\section{Materials and Methods}

\section{Animals}

Three rat strains were used for all experiments. The Long Evans strain (Charles River Labs) was used as the control for all experiments and exhibits normal retinal function and morphology. Homozygous $\mathrm{Tg} \mathrm{P} 23 \mathrm{H}-1$ and $\mathrm{Tg}$ S334ter-3 rats were acquired from Dr. Matthew LaVail at the University of California, San Francisco, CA. Homozygous animals for both models are albino as the Sprague-Dawley rat was used as the background strain. In order to circumvent effects of albinism, homozygous Tg animals were bred with Long Evans rats to create hemizygous Tg pigmented animals with one copy of the 
transgene and two normal rat rhodopsin genes. Hemizygous Tg animals are used in all experiments.

\section{In Vivo Experimental Surgery}

The animal is first anesthetized with a cocktail of anesthesia consisting of ketamine and xylazine administered at a dose of $\sim 105$ and $7.5 \mathrm{mg} / \mathrm{kg}$ respectively. Once deeply anesthetized, a custom catheter is surgically placed in the femoral vein to administer a booster cocktail at half the original concentration. The catheter is attached to a syringe pump to keep the animal at a constant plane of anesthesia for the duration of the experiment. Once the catheter is in place, the animal is placed in a stereotaxis (David Kopf Instruments) to immobilize the head. The skin over the skull is retracted by making a 1 inch incision down the midline. Any connective tissue is teased away from the skull to expose the sutures. Using the labdoidal, coronal and sagittal sutures as landmarks, a micro drill (Fine Science Tools, Heidelberg, Germany) is used to make an approximately $1 \times 1 \mathrm{~cm}$ opening in the skull. The dura is removed carefully and once exposed, the surface of the brain is vacuum aspirated to expose the surface of the SC. 


\section{In Vivo Electrophysiology}

Once the surface of the SC is exposed, a custom made lacquer coated tungsten electrode $(0.25-2 \mathrm{M} \Omega)$ is used to record extra-cellular activity in the SC. The margins of the SC are estimated based on large blood vessels that border the edges. The electrode is positioned over the first SC site using a set of manipulators (David Kopf Instruments) along the $X$ and $Y$ axis. Once in place, a hydraulic microdrive (David Kopf Instruments) is used to lower the electrode to the proper depth at a rate of $25 \mu \mathrm{m}$ per second. The best recorded responses were at a depth range between 150 and 400 microns. A clear bursting activity was heard if the electrode was lowered beyond this range, indicating the electrode had passed through the superficial visual layers of the SC. Data was sampled at 54 sites encompassing the entire exposed surface of the SC. The

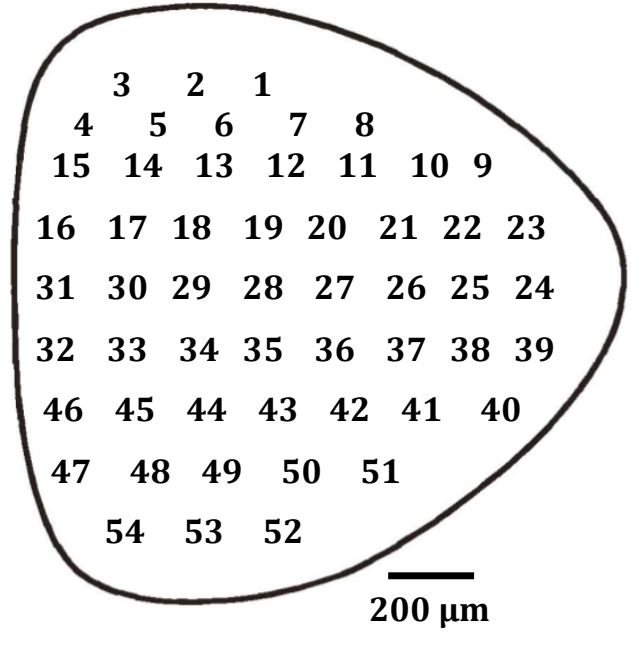

Figure 26. The 54 sites sampled across the dorsal surface of the SC. space between two adjacent sites is approximately $200 \mu \mathrm{m}$ (Figure 26).

Full field stimulation with a peak wavelength of $470 \mathrm{~nm}$ was presented using a hemi-field dome in a dim room. The stimulus intensity, duration (2s), and inter-stimulus interval (10s) was controlled using custom programs in LabView (National Instruments, Austin, TX). Signals were amplified through an XCell-3 Microelectrode 
Amplifier (FHC, Bowdoin, ME) and sampled at $10 \mathrm{KHz}$ using a Power1401 data acquisition unit (Cambridge Electronic Design, Cambridge, England). Data was collected and stored on a computer running Spike2 software (Cambridge Electronic Design, Cambridge, England).

\section{In Vivo Data Analysis}

Data was later analyzed offline using custom scripts in Spike2. Briefly, a threshold was set at 3.5 standard deviations above the average noise (spontaneous activity) levels and this was used to define the response (or lack of). The raw data was converted to a wave form based on the rate histogram (Figure 27). This wave form was then averaged across the $\sim 20$ trials and then analyzed for response parameters, including response area, amplitude, time to peak, and response duration. This was further broken down to the ON response and OFF response as the long 2 s stimulus allows the two responses to be clearly distinguished. Once the response parameters were quantified, statistical analysis was performed using Prism 6 (GraphPad, Inc., LaJolla, CA). 
A.

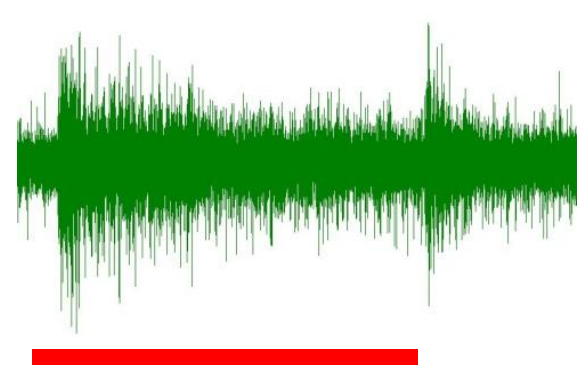

Figure 27. A. Example raw SC response trace. B. PSTH generated from the raw trace. C. Wave form converted from the PSTH in B. The response is defined as the time at which the trace crosses threshold (blue line in $\mathrm{C}$.) to the time it dips back below threshold. Threshold is defined as $\mathbf{3 . 5}$ standard deviations above the spontaneous activity. The red bar is the time the stimulus is on (2s).

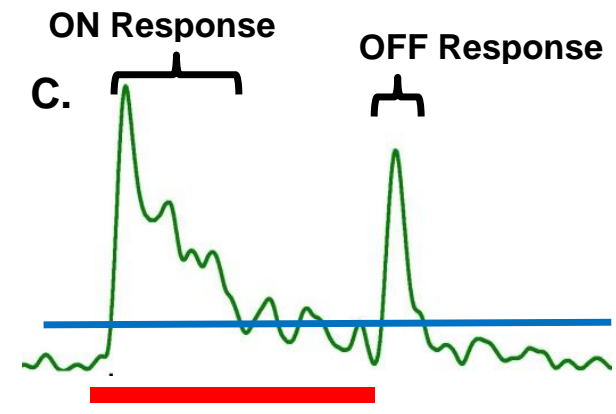

\section{In Vitro Preparation}

For multi-electrode array (MEA) recordings, animals were dark adapted for at least 1 hour prior to recording. Using infra-red goggles, animals were anesthetized with the same cocktail of ketamine and xylazine. Once deeply anesthetized, the animal was sacrificed via thoracotomy and the eyes enucleated. Under dim red light, the cornea and lens were removed and the retina dissected from the eye cup. The retina was then laid RGC side up and rinsed with oxygenated bicarbonate buffered Ringer's solution. A mixture of collagenase and hyaluronidase was then placed on the retina for 15 minutes to remove the vitreous. The retina was then rinsed three times, any remaining vitreous removed with fine forceps, and then quartered. One quarter was placed photoreceptor side against a culture membrane and then transferred to the recording chamber where it was placed RGC side against the electrodes. A 
platinum ring was then placed on top of the membrane but not the retina, so not to occlude any visual stimulus from the retina.

\section{In Vitro Electrophysiology}

The MEA (Multi-Channel Systems, Reutlingen, Germany) consists of a glass chamber with 60 platinum-iridium electrodes lining the bottom of the chamber. Each electrode is $30 \mu \mathrm{m}$ in diameter and the inter-electrode distance is $200 \mu \mathrm{m}$. Each electrode records extracellular spiking activity from the RGCs. Data is sampled at $10 \mathrm{kHz}$ and recorded with a MEA1060 system from MultiChannel Systems. The same full-field stimulus (470 nm, duration, intensity, isi, etc.) used for the in vivo experiments was used for these experiments. The data was collected using MC_Rack software (Multi-Channel Systems), band pass filtered between 80 and $3000 \mathrm{~Hz}$ and spikes were sorted into individual units manually using Offline Sorter (Plexon, Inc., Dallas, TX). Individual units with an amplitude less than $\sim 40 \mu \mathrm{V}$ were not used. Data was then analyzed using Neuroexplorer (Plexon, Inc., Dallas, TX).

\section{ON-OFF Index}

To compare the ON-OFF ratio between groups, an ON-OFF index value was calculated. This was done to normalize the data for comparison and ensure that the absolute values were not artificially creating differences between groups due to an experimental setup difference over time as this study was dependent on large age differences between animals, and hence long times between 
recording different groups. This value is calculated as follows; the OFF component is subtracted from the ON component and this is then divided by the sum of the same ON and OFF component:

\section{$\frac{\mathrm{ON}-\mathrm{OFF}}{\mathrm{ON}+\mathrm{OFF}}$}

This results in a possible range from 1 to -1 , where 1 represents a completely ON dominated response, -1 represents a completely OFF dominated response and 0 would be equal responses between the two components.

\section{Results}

As photoreceptors are lost, there is a shift from ON to OFF dominated SC responses in $\mathrm{Tg} \mathrm{P} 23 \mathrm{H}-1$ rats

I recorded the visual responses in the SC of control rats as they aged (50 to 300 days). In the SC, responses to a $2 s$ stimulus evoked a response at both light onset and at light offset. In control rats, SC responses show both an ON and OFF response to visual stimuli with a slightly larger ON response on average. This response profile does not change with age in WT rats. As no changes were seen in WT rats, in all of the comparisons with Tg rats, WT data were combined into one control group.

Consistent with morphological observations (Kolomiets et al., 2010), the cone-driven visual responses in hemizygous Tg P23H-1 RGCs declined with age up to P550 (Sekirnjak et al., 2009). My data also show visually evoked responses in the SC through $\mathrm{P} 300$ in hemizygous $\mathrm{Tg} \mathrm{P} 23 \mathrm{H}-1$ rats, my oldest 
group evaluated. In addition to the overall loss of visual function, I found a surprising change in the balance between the ON-OFF responses in the SC in $\mathrm{Tg} \mathrm{P23H-1} \mathrm{rats} \mathrm{when} \mathrm{compared} \mathrm{to} \mathrm{WT.} \mathrm{In} \mathrm{Tg} \mathrm{P23H-1} \mathrm{animals} \mathrm{up} \mathrm{to} \sim \mathrm{P} 90, \mathrm{SC}$ responses are balanced between ON and OFF and are relatively similar to WT. From $\sim \mathrm{P} 120$ the $\mathrm{Tg} \mathrm{P} 23 \mathrm{H}-1$ responses in the SC shifted towards OFF dominated and the shift became more pronounced with age and progression of photoreceptor degeneration (Figure 28). This finding had not been previously reported in this RP model; in fact a lack of change has been reported (Sekirnjak et al., 2009). At approximately P300, where the total ON response has decline to $\sim 25 \%$ of $\mathrm{WT}, \mathrm{Tg} \mathrm{P} 23 \mathrm{H}-1 \mathrm{SC}$ responses are strongly OFF-dominated and the OFF response has increased to $250 \%$ over the WT OFF response (Figure 29). The total response depends on both response amplitude and duration, and changes in either of these parameters could cause this overall response change. I examined both of these parameters and Figure 30 plots the changes in duration and amplitude of the SC ON and OFF responses as a function of age. 

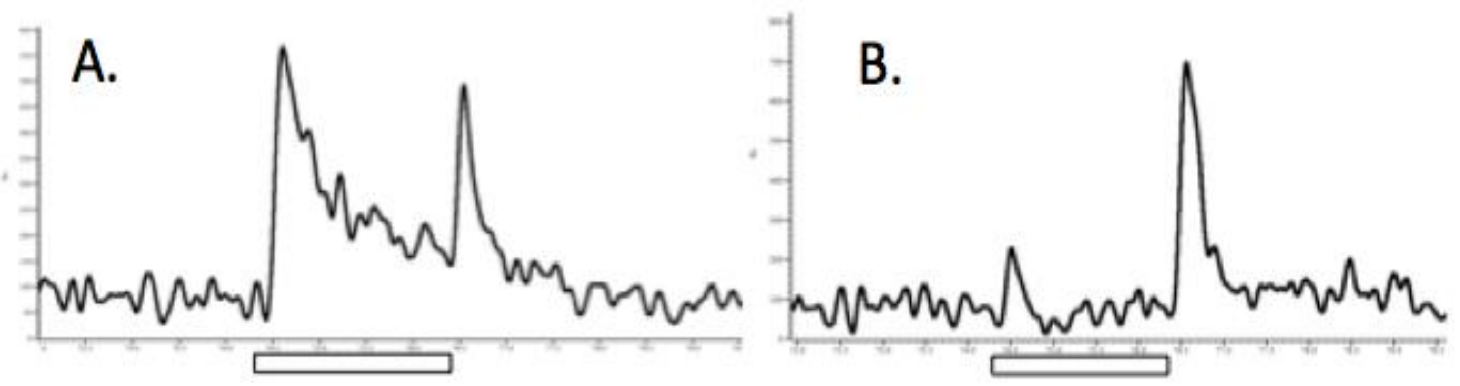

Figure 28.

A: Typical visually-evoked response in the SC of a WT rat.

B: Typical visually evoked response in the SC of $\mathrm{P} 300 \mathrm{Tg} \mathrm{P} 23 \mathrm{H}-1$ rats. The white bar beneath each PSTH represents the duration of the visible stimulus. There is a large On response and a smaller Off response in WT SC. In contrast, the response in P300 Tg P23H rats shows a strongly Offdominated response that has developed with age.
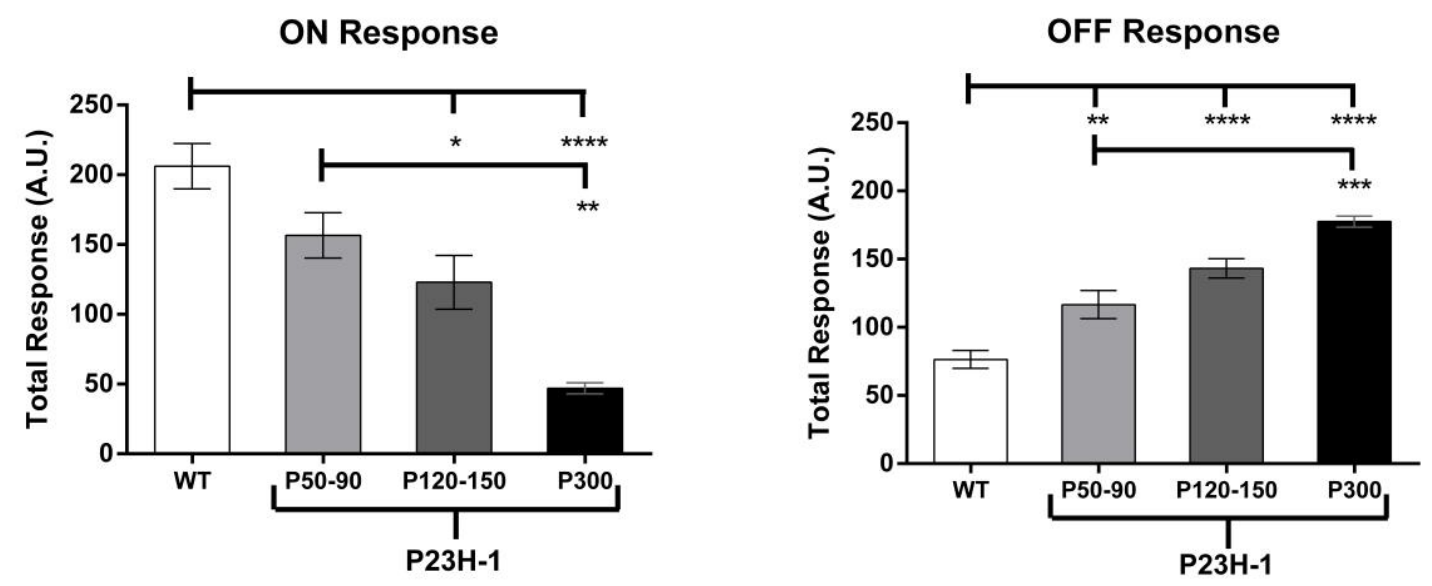

Figure 29. Comparison of the On and Off total SC response in $\mathrm{Tg} \mathrm{P} 23 \mathrm{H}-1$ rats as they age compared to WT rats. No age related changes were seen in WT rats, therefore all WT animals were grouped for this comparison. * $p<0.05$, ${ }^{\star *} p<0.01,{ }^{\star * *} p<0.001,{ }^{* \star \star *} p<0.0001$. WT $n=13, \mathrm{Tg}$ P23H-1 $n=6$ for each group 
A.

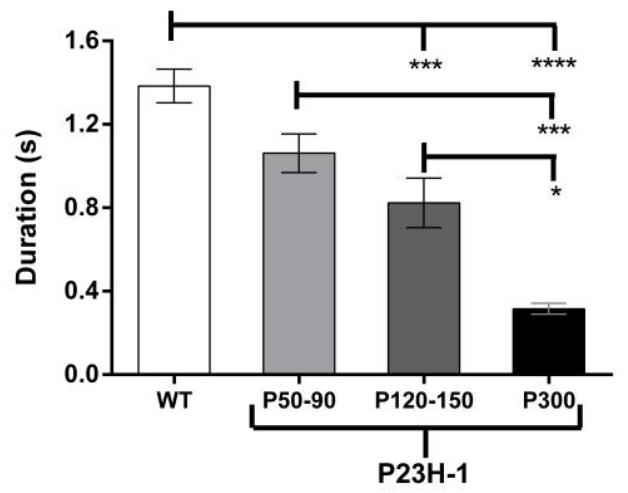

B.

ON Response

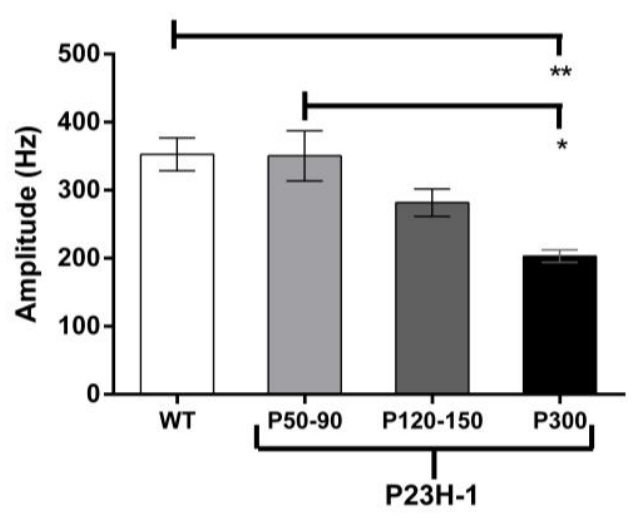

OFF Response

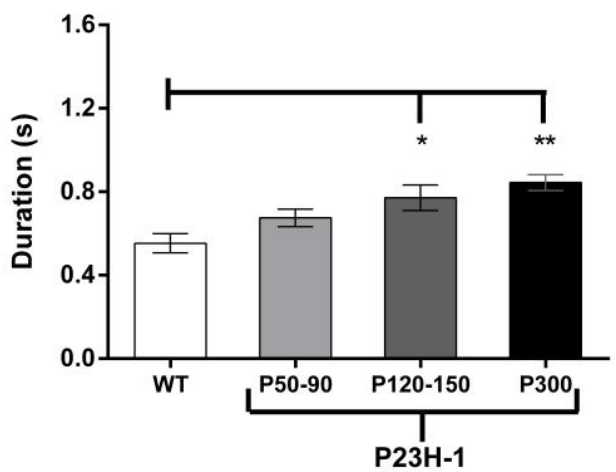

OFF Response

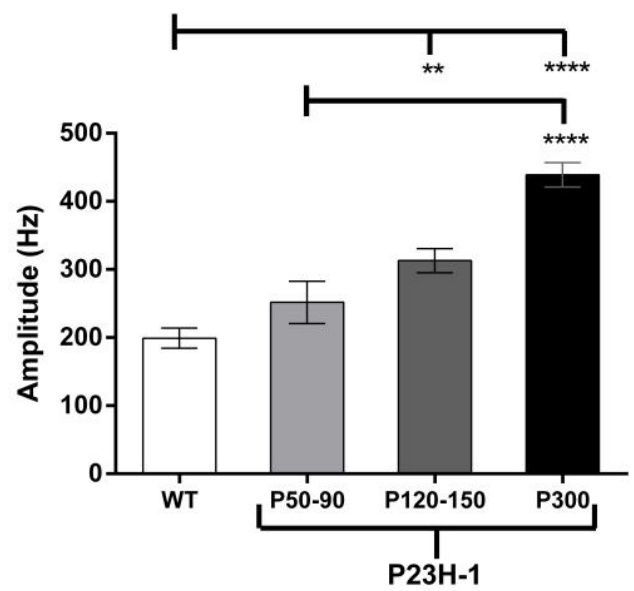

Figure 30. A. ON and OFF response duration in WT vs. Tg P23H-1 rats as they age. $\mathrm{B}$. ON and OFF response amplitude in WT vs. $\mathrm{Tg} \mathrm{P} 23 \mathrm{H}-1$ rats as they age. ${ }^{\star} p<0.05,{ }^{\star \star} p<0.01,{ }^{\star \star \star} p<0.001,{ }^{\star \star \star \star} p<0.0001$. WT $n=13$ Tg P23H-1 $n=6$ per group

The results suggest that the loss of the $\mathrm{ON}$ response is due mainly to a loss of response duration. In contrast, the increase in the OFF response is driven by an increase in response amplitude. This indicates that the ON pathway input to the SC initially becomes more transient and then loses overall excitatory input or there is a change in the SC circuitry as a result of a loss of excitatory input from the retina. The OFF response peak amplitude significantly increases, which is 
most likely to result from a loss of tonic inhibition from the declining ON pathway either at the level of the retinal input or within the SC circuit.

OFF dominated SC responses arise early and do not change in Tg S334ter-3 rats

When compared to the $\mathrm{Tg} \mathrm{P} 23 \mathrm{H}-1$ rat, the $\mathrm{Tg}$ S334ter-3 mutation results in a much faster degeneration of photoreceptors. This includes a decline in both rod and cone driven responses in the SC. The functional consequences of this degeneration were equally rapid. In Tg S334ter-3 rats, SC responses were already OFF dominated at the youngest age I recorded, P25 (Figure 31; Note: it is very difficult to record from rats younger than P25 in vivo). This difference between the two models is clearly evident with the aggressive degeneration present in the Tg S334ter-3 model.
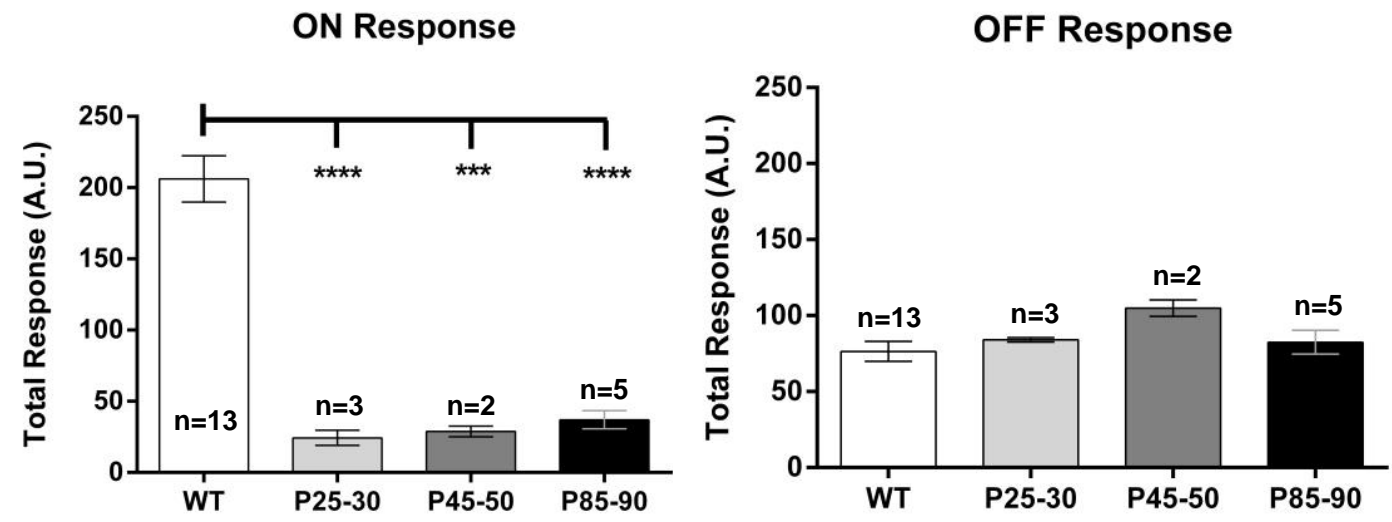

Figure 31. Comparison of the $\mathrm{ON}$ and OFF total $\mathrm{SC}$ response in $\mathrm{Tg}$ S334ter-3 rats. In contrast to the $\mathrm{Tg} \mathrm{P23H}-1$ rats, there were no age related changes in the Tg S334ter-3 rats. ${ }^{*} p<0.05,{ }^{* \star} p<0.01,{ }^{\star \star *} p<0.001$, ****P<0.0001

Similar to previous publications, I found that by $\sim P 180$, all visual responses in the SC are lost. There also were no significant changes in the ON or OFF response 
characteristics as the animals aged in contrast to $\mathrm{Tg} \mathrm{P} 23 \mathrm{H}-1$ rats. While the OFF response increased in Tg P23H-1 rats, this was not the case in Tg S334ter-3 rats. The OFF response was not significantly different from WT in Tg S334ter-3 rats at any age. Combined with the lack of change in the ON pathway with age, this may indicate a sort of functional steady state within the retina until all visual responses are lost. In addition, this may mean only neurons within the ON pathway need to be targeted for treatment, while both the ON and OFF pathway in $\mathrm{Tg} \mathrm{P} 23 \mathrm{H}-1$ rats may need to be treated to address their respective changes. Figure 32 plots the ON-OFF index values for the Tg S334ter-3 groups. When compared to the profile of the $\mathrm{Tg} \mathrm{P} 23 \mathrm{H}-1, \mathrm{Tg}$ S334ter-3 rats have a similar profile to the oldest $\mathrm{Tg} \mathrm{P} 23 \mathrm{H}-1$ rats with very OFF dominated responses in all age groups.

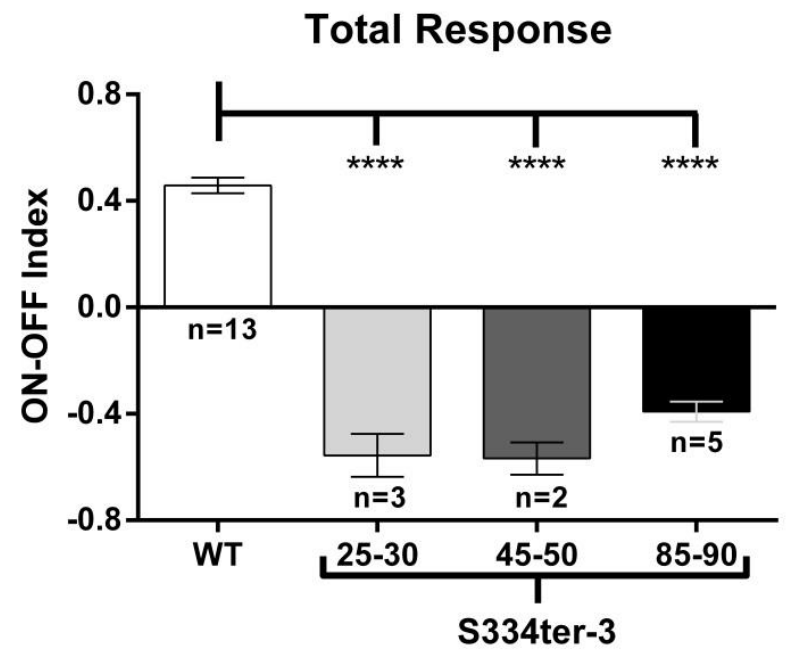

Figure 32. The ONOFF index for Tg S334ter-3 rats shows strongly OFF dominated responses, similar to P33 Tg P23H-1 rats but does not change as a function of age. ${ }^{* \star * *} p<0.0001$ 
The loss of ON responses is not specific to the SC as $\mathrm{Tg} \mathrm{P} 23 \mathrm{H}-1$ rat retina shows the loss of $O N$ responses in vitro

To determine if the shift towards OFF dominated responses can be the consequence of changes in the input to the SC, I evaluated the spontaneous and visually evoked responses in Tg P23H-1 rat RGCs using an MEA in vitro. MEA recordings were made from both WT and from Tg P23H-1 RGCs at P35 and P300. Only $60 \%$ of the RGCs in the $\mathrm{P} 300 \mathrm{Tg} \mathrm{P} 23 \mathrm{H}-1$ were visually responsive compared to $96.8 \%$ and $98.7 \%$ for $\mathrm{P} 35 \mathrm{Tg} \mathrm{P} 23 \mathrm{H}-1$ and WT rats respectively. In WT rats, the RGC profile is $37 \%$ ON RGCs, $41 \%$ OFF, and $21 \%$ ON-OFF RGCs. Only one RGC (2\%) was unresponsive to visual stimuli in WT rat retina. The P35 Tg P23H-1 retinas showed a similar RGC response profile; there were relatively equal proportions of ON (37\%) and OFF RGCs (36\%), a smaller proportion of ON/OFF RGCs (25\%) and very few visually non-responsive RGCs (3\%). In the P300 Tg P23H-1 retinas the portion of ON or ON-OFF RGCs (2\%) was greatly reduced, while OFF type RGCs were still abundant in $\mathrm{P} 300 \mathrm{Tg} \mathrm{P} 23 \mathrm{H}-1$ retinas (Figure 33). In addition, there were a large proportion of non-responsive cells in the $\mathrm{P} 300$ retinas $(40 \%)$. Also of note, the total percent of OFF cells is relatively similar across the three groups, at $\sim 40-45 \%$. This indicates that RGCs are not changing their response characteristics, i.e. ON cells are not becoming OFF cells during rewiring of the retinal circuitry. It appears that the response to light onset is declining and producing a decrease in RGCs with either ON or ON-OFF responses and that as this occurs there is an increase in visually non-responsive cells, while the OFF pathway remains robust. 
WT

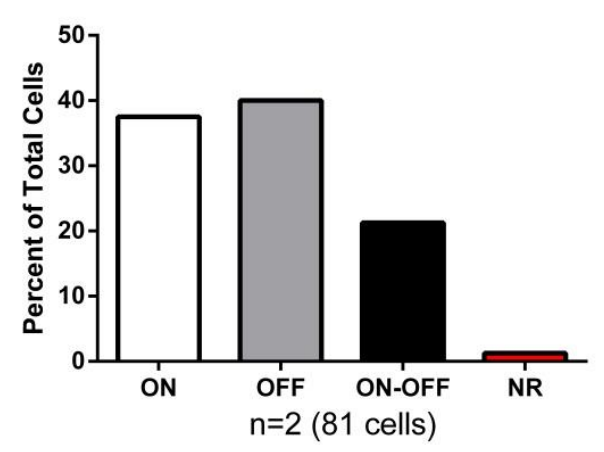

P300 P23H

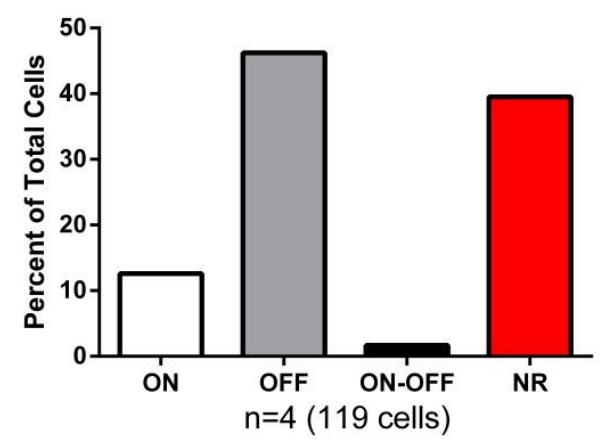

P35 P23H

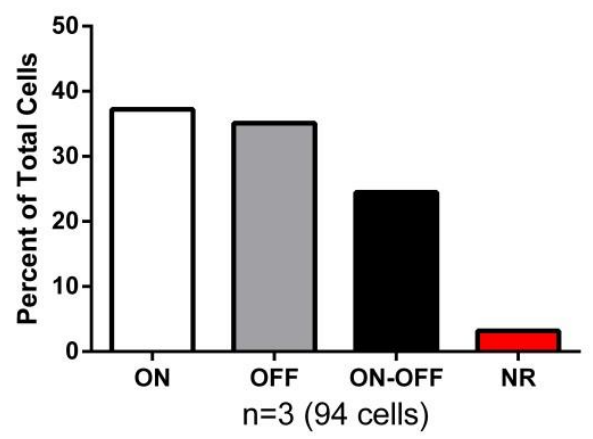

Figure 33. The cell class profile for WT, P35 and P300 Tg P23H-1 retinas. A clear change was evident in the P300 animals while the P35 group has a very similar distribution as WT animals. NR = non-responsive RGCs

Differential changes in the spontaneous activity of ON and OFF RGCs in Tg $P 23 \mathrm{H}-1$ rat retina.

In WT rat retina, no changes are evident in the spontaneous activity of any RGC class as the animals age. The frequency of action potentials does not increase with age in WT rats. No inherent peak frequency is present in the spontaneous firing rate either. In many retinal dystrophy models spontaneous activity often changes in frequency and/or pattern. For example, in mouse models of RP (Stasheff 2008,Trenholm et al., 2012) and congenital stationary night blindness (CSNB, Demas et al., 2006), changes in spontaneous activity of RGCs have been described in the form of bursting activity and increased overall activity. In RP models, these changes are correlated with the decline of 
photoreceptors. At P300, Tg P23H-1 OFF RGCs exhibit spontaneous activity with a significant rhythmic component, defined by a peak F0 component when a fast Fourier transformation (FFT) was performed. The remaining ON and ONOFF RGCs did not show a significant rhythmic component (Figure 34).

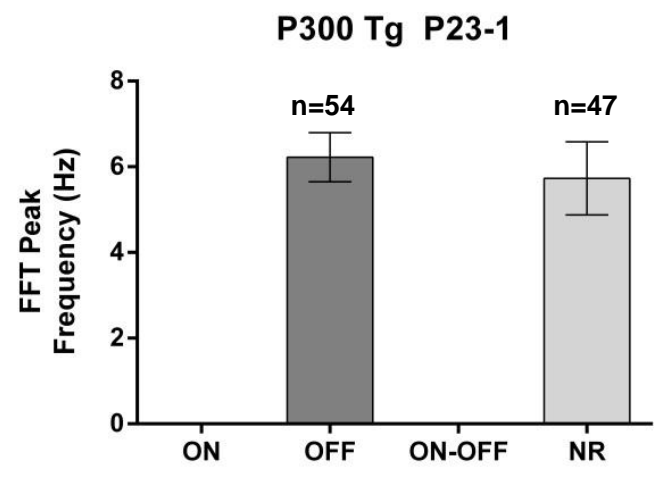

Figure 34. F0 peak of the fast Fourier transform in P300 Tg $\mathrm{P} 23 \mathrm{H}-1$ retinal cell classes. Only OFF cells and NR cells exhibited burst activity as evidenced by the F0 component.

When comparing the peak of the FFT, which shows the primary rhythmic frequency if there is rhythmic firing, OFF RGCs had an average fundamental peak at $6.22 \mathrm{~Hz}$ compared to $2.92 \mathrm{~Hz}$ for P35 Tg P23H-1. Non-responsive P300 RGCs also showed a fundamental peak at $5.89 \mathrm{~Hz}$, but this was not significantly lower than OFF cells. This suggests the same mechanism is driving the bursting activity in these cells. ON and ON-OFF responsive cells never had any rhythmic activity, evident by the lack of an F0 peak in the FFT for those cells. This may indicate differences in circuitry that lead to an early onset of rhythmicity in OFF cells even though they are still receiving input, while ON or ON-OFF cells do not exhibit rhythmic bursting until they become non-responsive to visual stimuli.

OFF RGCs in both P35 and P300 Tg rats trend towards an increase in spontaneous activity, with an average firing rate of 9.97 and $11.07 \mathrm{~Hz}$ respectively. However, this did not reach significance compared to WT OFF cells at $6.61 \mathrm{~Hz}$. There was a trend for a drop in ON RGC spontaneous activity for 
both Tg groups compared to WT, but again this was not a significant difference. Sekirnjak et al. (2011) reported an increase in OFF RGC spontaneous activity but no change in $\mathrm{ON}$ activity as $\mathrm{Tg} \mathrm{P} 23 \mathrm{H}-1$ rats age and this data has a similar trend.

\section{Discussion}

My data differs from previously published results describing RGC function in the Tg P23H-1 rat (Sekirnjak et al., 2011). In light of other models of RP that do show a shift towards OFF dominated responses (rd1, rd10, RCS rat), they tested the $\mathrm{Tg} \mathrm{P} 23 \mathrm{H}-1$ rat and concluded that there was no change in the ONOFF balance. My data show there are functional changes in both $\mathrm{Tg} \mathrm{P} 23 \mathrm{H}-1$ and Tg S334ter-3 rat models of RP. While the Tg P23H-1 rat showed a progressive shift from ON to OFF dominated SC responses, SC responses in the Tg S334ter3 rat were OFF dominated from the earliest age. Even though the two models are different in this respect, there is a common loss of the ON pathway while the OFF pathway remains robust. This change was very robust in my experiments so this may be a consequence of a different experimental setup. Their stimulus was white noise, which consists of a randomly flickering checkerboard of small squares, while my stimulus was full field.

The MEA data for $\mathrm{Tg} \mathrm{P} 23 \mathrm{H}-1$ suggests that the shift to OFF dominated responses in the SC is due to a change in input from RGCs. This does not eliminate the possibility that there are reactive changes in the SC however. In fact, functional and structural changes in the SC of RCS rats have been reported 
previously. Vugler and Coffey (2003) reported the loss of calretinin and parvalbumin immunoreactivity in SC neurons of RCS rats Pothecary et al. (2005) reported altered SC expression of iGluR receptors in RCS rats that led to changes in SC responses compared to wild type rats. These examples may be mutation specific but it is likely that the SC (and other areas of the visual system) exhibits reactive changes during the degenerative process that is not mutation specific.

This functional change from ON to OFF dominated responses appears to be a consequence of photoreceptor degeneration in general, rather than specific to certain RP models. This is the fourth description of such a change and the third mutation (Mertk, Pde6, and now rhodopsin) in which this change is occurring. Similar to the $\mathrm{Tg}$ S334ter-3, the rd1 (Pde6 mutation) is a very rapid decline, yet still shows a similar preferential loss of the ON pathway. It could be argued that this may be specific to rodent models since all of the above examples are either rat or mouse but unpublished data from the McCall laboratory shows evidence of a similar change in a pig model of RP (also the $\mathrm{P} 23 \mathrm{H}$ mutation). If this is something universal to photoreceptor degeneration, it may be possible to exploit this change in the clinic to either diagnose early stages of RP or AMD and also to assess the speed of disease progression, perhaps looking at changes in threshold responses of patients to light increments and decrements. Renner et al. (2006) reported that there was a larger decrease in the ON ERG response vs. OFF response in humans with various retinal dystrophies thought not in RP patients. This was a small sample but does 
support the possibility that a differential change in the ON vs. OFF pathway is detectable in the clinic.

Since this functional change appears across multiple models with different mutations (rd1-Pde6, RCS-Mertk, P23H/S334ter -rhodopsin), a more universal explanation is likely to account for these changes. My hypothesis is that the loss of expression of mGluR receptors is a protective mechanism for ON BCs. As photoreceptors are lost, glutamate release onto the terminals of the horizontal and $\mathrm{BC}$ dendrites must decline. Since $\mathrm{ON} B C$ s respond in a sign inverting manner to glutamate, the absence of glutamate may keep these ON BCs in a depolarized condition. If this is the case and there are no compensatory mechanisms, the time the TrpM1 channel is in its open state should increase and the constant depolarized state should increase intracellular calcium potentially leading to excitotoxicity and eventually cell death if the depolarization is not corrected. As there is no precedence for a selective cell death of ON vs. OFF $\mathrm{BCs}$, it is likely that the ON BCs may eliminate expression of the mGluR6 receptor either by a reduction in trafficking to the cell surface or down regulation of expression in general. In contrast, OFF BCs express ionotropic glutamate receptors, which are sign conserving so the loss of glutamate is a lack of depolarization. In this case, there would be no excitotoxicity that would cause OFF BCs to stop expressing iGluR on their dendritic tips. In addition, the nob mouse does not show the same change glutamate receptor expression (Ball et al., 2003) even though there is a lack of response from ON BCs. The nob mouse is a model for congenital stationary night blindness in which the protein 
nyctalopin is mutated, which is required for the localization of TrpM1 channels to the ON BC dendritic tips. Photoreceptors in the nob mouse are still functional, as evidenced by the presence of an a-wave, so BCs still receive input but this input no longer results in membrane voltage changes in ON BCs. This suggests that the mechanism depends on input from photoreceptors and the presence of a functional TrpM1 channel for expression to change. Without functional TrpM1 channels, there is no depolarization in ON BCs and therefore no excitotoxicity in nob. Therefore, the mechanism that reduces mGluR6 expression is never initiated in the ON BCs of the nob mutant. I believe this supports the idea that the lack of input from photoreceptors is causing the reactive changes seen in RP models.

These functional changes also have important implications for future treatments. For example, if stem cells are able to integrate into a host retina, how can we be certain that the retina will respond properly if there is significant remodeling in structure and function? If this change does represent a modification in the expression of glutamate receptors, as has been previously reported, then the release of glutamate from newly integrated photoreceptors may be ineffective. It's possible that a feedback mechanism may exist if photoreceptors are reintroduced to the system, causing the BCs to express their normal glutamate receptors again but this has not been reported to occur. 


\section{CHAPTER IV}

\section{IMPLANTATION OF RETINAL PROSTHETIC DEVICES IMPARTS A FUNCTIONAL NEUROPROTECTIVE EFFECT}

\section{Introduction}

When a cure is not available, an important strategy in treating neurodegenerative disease is to slow its progression. The mPVA device has been observed to exhibit neuroprotective effects by preservation of photoreceptor numbers (Pardue et al., 2005) and as light evoked responses recorded in the SC (DeMarco et al., 2007). There is evidence that electrical stimulation, in general, provides a neuroprotective effect with an increase in ONL thickness and ERG awave and b-wave amplitude (Morimoto et al., 2005, Morimoto et al., 2007, Ni et al., 2009, Morimoto et al., 2012). The question remains however, does preservation have functional implications that have not been previously tested? In cases where there was preservation, the benefit was often transient, delaying degeneration for a short time (1-2 weeks) (Morimoto et al., 2007, Ciavatta et al., 2009). This does not preclude the possibility that an effect on the retina still exists after photoreceptors are lost. This could be in the form of preservation of the remaining cellular layers (INL, RGC), receptor expression (mGluR, Glycine, 
GABA), or RPE structure as RPE cells often invade other cellular layers during the degenerative process. The possibility of a prosthesis-mediated neuroprotective effect has important implications for the timing of surgery in a patient. If there is a long-term functional preservation of visual responses or if cells or the structure of the retina is preserved, it may mean implantation at an earlier stage of degeneration may be beneficial. Alternatively, if there is no protective effect, surgery may be best left until late stages of the disease when the risk to the patient is minimized. Although I did not perform a stimulation protocol in the implanted rats, it is possible that the low level of current generated by the implant with ambient lighting conditions may stimulate the retina enough to support neuroprotection.

\section{Experimental Goal}

The goal of these experiments was to determine if there is any effect on the visually-evoked response recorded in the SC when a prosthetic device is present. With the ON-OFF response changes described in Chapter III for both $\mathrm{RP}$ models, a neuroprotective effect is defined here as a significant increase in the function of the ON pathway. In WT, both the ON and OFF pathways are functional and stable with age. In both models, the OFF pathway dominates the degenerate retina, either early in the process ( $\operatorname{Tg}$ S334ter-3) or as the animals age $(\mathrm{Tg} \mathrm{P} 23 \mathrm{H}-1)$. The implication for degenerative diseases, if this is a common symptomatic change, is that without a functional ON pathway, treatment options may be limited in their potential for normal vision. 


\section{Materials and Methods}

\section{Animals}

Three groups of Tg P23H-1 and Tg S334ter-3 animals were used in these experiments. One group of $\mathrm{Tg} \mathrm{P} 23 \mathrm{H}-1$ was implanted with bPVA devices and tested. Two groups of Tg S334ter-3 animals were tested; one group implanted with bPVA devices and the other implanted with mPVA devices.

\section{Implantation Surgery}

The same protocol was used as described in Chapter II. Animals were implanted at $\sim$ P35 for both Tg models. This age is very early in the degenerative process for the $\mathrm{Tg} \mathrm{P} 23 \mathrm{H}-1$ rat when responses are similar to WT. Only a small loss of ONL thickness is evident at this age, with $\sim 12$ rows of nuclei remaining vs. $\sim 15$ rows in WT rats. The Tg S334ter-3 responses were already significantly different from WT at this age, but this is as early as the rats will easily tolerate anesthesia and surgery to make a full recovery. In addition, there are still 3-5 rows of nuclei at this age, allowing some degeneration to still take place before experiments were carried out. Tg S334ter-3 were $\sim \mathrm{P} 75$ and Tg P23H-1 were $\sim$ P150 at the time of recording since these time points represent when retinal function is significantly different from WT rats for both groups.

\section{In Vivo Electrophysiology}

These experiments borrow elements from both Chapter II and III for the electrophysiology. Data was collected and analyzed in a similar fashion using an XCell3 Microelectrode Amplifier (FHC) into a Power 1401 data acquisition unit 
(CED). Data was collected and stored on a computer running Spike2 (CED) and analyzed offline. To assess the function as it related to the region of implant influence, the visually evoked responses were mapped using the same hemi-field LED stimulus for the right eye and the IR evoked responses were mapped using the IR laser system. This allowed for a retinotopic map of both visual and IR evoked responses and for regions under the influence of the prosthesis to be compared to regions outside the influence of the prosthesis. To examine whether there is a trophic effect, $\mathrm{Tg} \mathrm{P} 23 \mathrm{H}-1$ rats were implanted with a bPVA device. The $\mathrm{Tg} \mathrm{P} 23 \mathrm{H}-1$ group was implanted with bPVA devices for this specific set of data while Tg S334ter-3 data was gathered from animals implanted for data in Chapter II. I recorded and characterized the prosthetic and visually evoked responses in the SC and examined whether there was evidence of a neurotrophic effect by comparing the visually evoked responses within animals depending on the proximity to PVA influence. In addition, these responses were compared with responses of non-implanted controls whose ages were matched to the age at the endpoint of implantation ( P140-150). I used full field visible stimuli and the IR laser and recorded these evoked responses in a systematic map across the SC dorsal surface. I compared visually evoked responses, which changed significantly with disease progression (see Chapter III for detailed analysis): the shift in the ON-OFF index and spontaneous activity levels. 


\section{Results}

Implantation of a bPVA device imparts a neuroprotective effect in the $\mathrm{Tg} \mathrm{P} 23 \mathrm{H}-1$ rat retina

In the $\mathrm{Tg} \mathrm{P} 23 \mathrm{H}-1$ rat model, there is a prolonged decline in visually evoked responses, a change in spontaneous activity of OFF and non-responsive (NR) RGCs (bursting activity), as well as a shift from ON dominated responses to

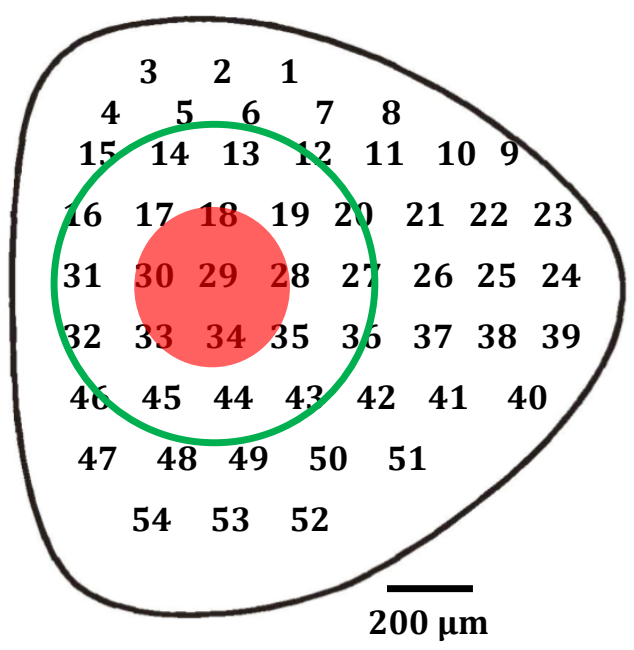

Figure 35. Schematic diagram of IR, Near and Far site designation. The red circle represents the IR responsive sites, the green circle excluding the red circle represents near sites $(<400 \mu \mathrm{m})$ and outside the green circle are far sites $(>400 \mu \mathrm{m})$.
OFF dominated responses (See Chapter III). In vitro recordings from RGCs in these rats showed similar changes with the loss of ON responsive RGCs, indicating that the changes arise from altered retinal signaling and input to the SC. To determine if there was a functional neuroprotective effect of implantation, I divided the SC into regions based on how close they were to the SC area

where IR-evoked responses could be recorded. Figure 35 shows an example of how these areas were calculated. Comparisons were made between areas that showed direct IR-evoked responses, areas adjacent to $(<400 \mu \mathrm{m})$ PVAresponsive sites, and areas far away from the area of PVA influence $(>400)$. Figure 36 shows an example of the two topographic responses maps of a $\mathrm{Tg}$ $\mathrm{P} 23 \mathrm{H}-1 \mathrm{SC}$ at $\mathrm{P} 140$. The maps were created from the IR-evoked responses 
(black and white) and visually evoked response plotted as the ON-OFF indices (color map with peak index value $=$ red and lowest index value $=$ blue). The large map shows the superimposition of the two individual maps. In the region of overlap, the ON-OFF index is highest for this animal, indicating there may be a correlation between the region of PVA influence and the function of the ON pathway.

To evaluate whether this neurotrophic effect was confined to the are directly in contact with the implant or a paracrine effect that could encompass the entire retina, I compared the average ON-OFF index within IR responsive in the Tg P23H-1 SC sites to SC sites adjacent $(<400 \mu \mathrm{m})$ or distal $(\geq 400 \mu \mathrm{m})$ to this area in P150 animals. Figure 37 plots the ON-OFF index in relation to the PVA influence and shows that SC responses shift significantly towards balanced ON and OFF responses. Regardless of their distance from IR responsive areas, the areas outside of the influence of the device remained OFF dominant and did not differ significantly from one another. This indicates that the neurotrophic effect was limited to regions that are directly under the influence of the PVA, rather than a global effect. 

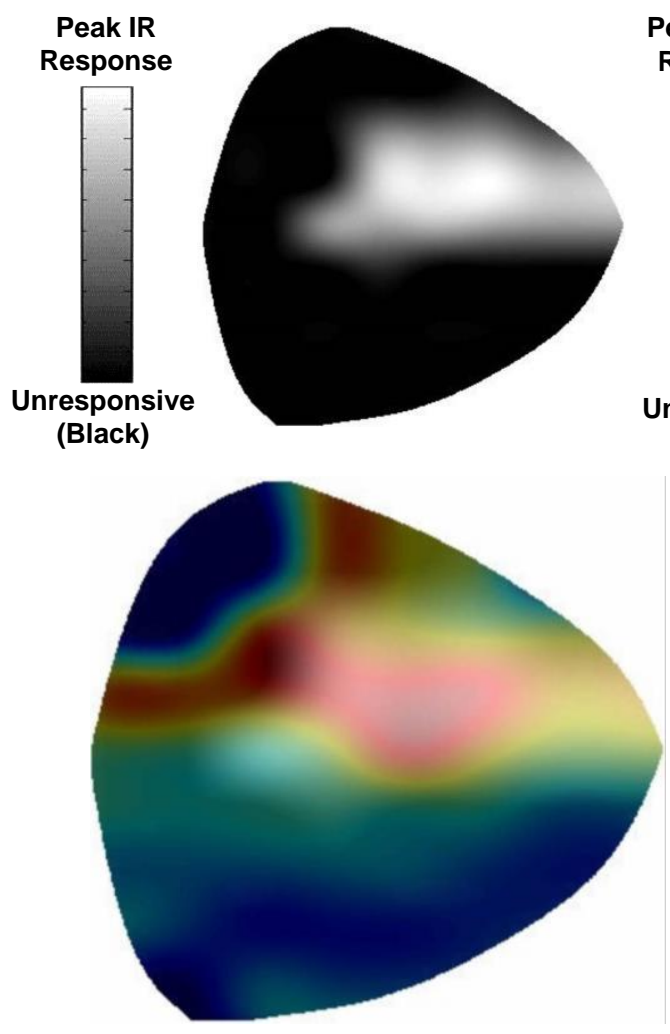

P23H with bPVA Device

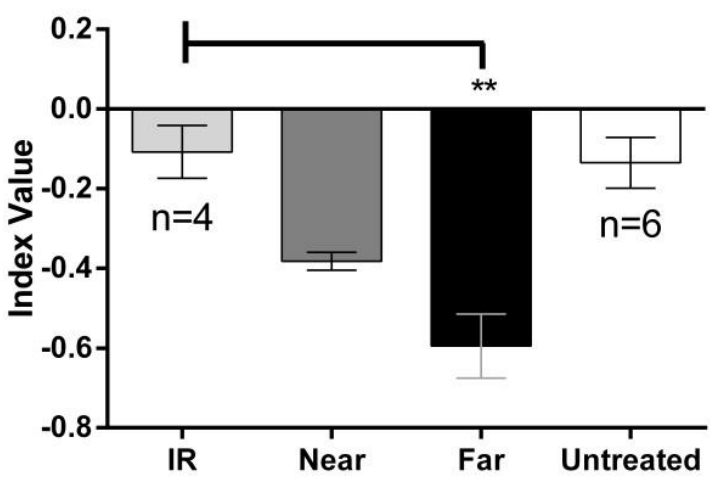

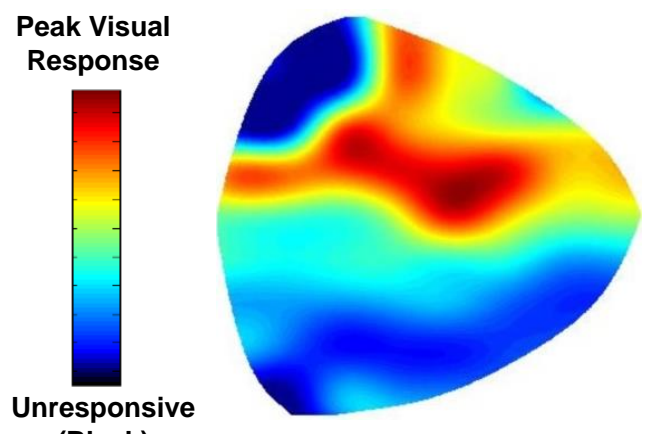

(Black)
Figure 36. Example SC topographic maps from the same $\mathrm{Tg} \mathrm{P} 23 \mathrm{H}-1$ animal. The black and white map represents IR responsive sites in white. The color map represents $\mathrm{ON}$ OFF index values, with the highest index values in red and low values in blue. The maps were then superimposed to show the relationship between the IR responsive region and the ON-OFF index value.
Figure 37. Comparison of the ON-OFF index and topographic relationship between IR responsive sites (IR), sites adjacent to but not more than $400 \mu \mathrm{m}$ from the IR responsive area (Near), and sites greater than $\mathbf{4 0 0} \mu \mathrm{m}$ from the IR responsive region (Far). .In bPVA implanted Tg P23H-1 rats, regions under the influence of the implant were significantly different than regions outside the influence of the prosthesis. ${ }^{* \star} p<0.01$

As described previously (Chapter III), the ON-OFF index depends on both peak amplitude and duration of the ON and OFF response. With this 
dependence, it is possible that rather than a protective effect on retinal function, the effect could result from a decrease in the OFF response amplitude or duration rather than a protective effect on the retina. To evaluate how the presence of the prosthetic changes these aspects of the response, I compared the amplitude and duration of the ON and OFF SC response. Figure 39 plots the ON and OFF duration and peak responses as a function of region and shows a significant increase in the total response of the ON response while the OFF response was similar across areas. This suggests that the neurotrophic effect of the device is selective to the ON pathway. The untreated Tg group was not significantly different than the IR responsive areas. This may suggest there is no neuroprotective effect. However, this can be interpreted in different ways and is addressed within the discussion section below.

Figure 38 shows that there was a significant increase in the amplitude of the response and a trend for the duration of the SC ON response to increase. In contrast, neither component of the OFF response was different. Taken together, there was a significant effect on the ON response in areas under the influence of the device while no change was seen in OFF responses regardless of the position relative to the bPVA. Spontaneous activity was also compared to determine if there was any effect on the firing rate. There was no effect across groups with implants, but the spontaneous activity of all regions of the SC was significantly lower than that of untreated, age-matched controls (Figure 39). It is difficult to say if this represents a protective effect as the spontaneous activity has been reported to change in $\mathrm{Tg} \mathrm{P} 23 \mathrm{H}-1$ rats depending on the $\mathrm{RGC}$ cell type 
(Sekirnjak et al., 2009). ON cells were reported to decrease their firing rate while OFF cells increase their spontaneous activity. Since the experiments outlined here are recording multicellular SC activity, it is impossible to know the functional class that has input to a particular area of the SC. Therefore, it cannot be determined if these changes are being mitigated by the PVA or if this was caused by another factor, such as variance between litters (implanted animals tended to be from the same litter).

A.

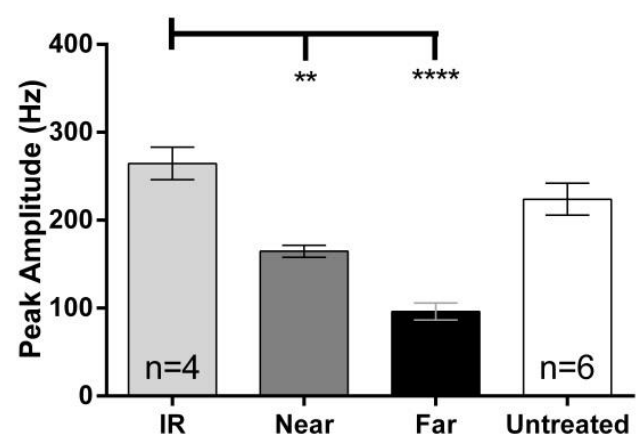

B.

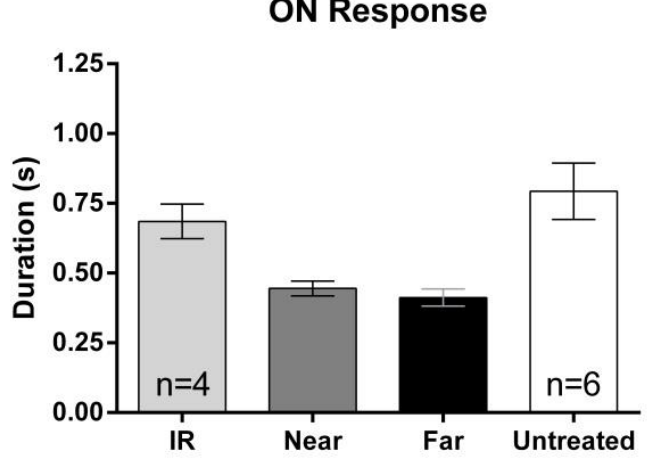

OFF Response

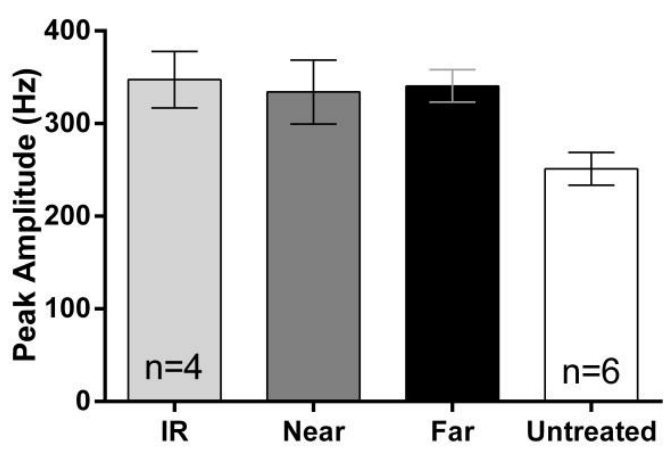

OFF Response

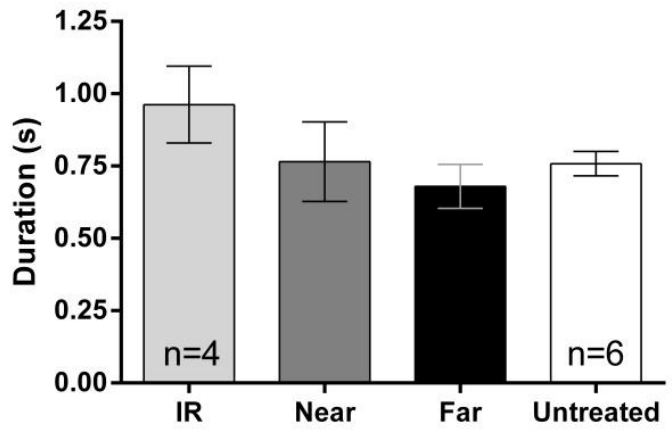

Figure 38.

A. Tg P23H-1 peak amplitude comparison for the ON (left) and OFF (right) response. ${ }^{\star *} p<0.01,{ }^{* \star \star *} p<0.0001$

B. Response duration comparison. No groups were significantly different from one another. 


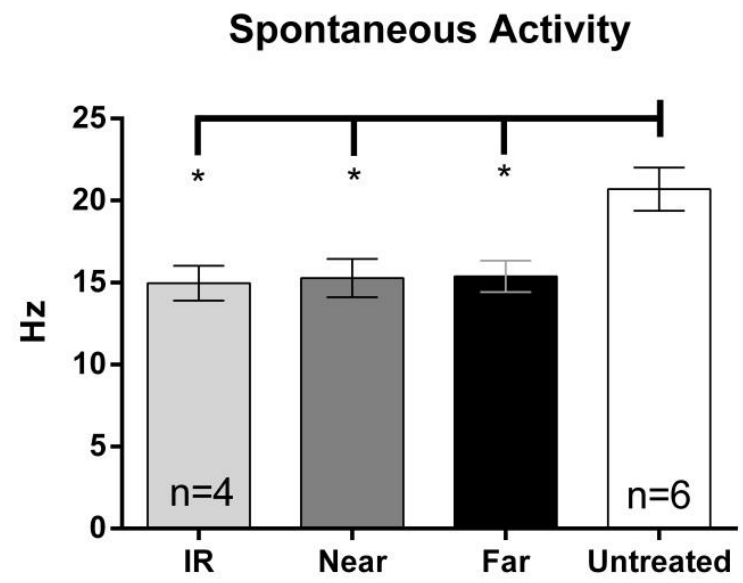

Figure 39. Comparison of the spontaneous $\mathrm{SC}$ activity in $\mathrm{Tg}$ $\mathrm{P} 23 \mathrm{H}-1$ rats as a function of implant influence. No region of the SC had any change in spontaneous activity but all regions had lower activity than untreated rats. ${ }^{*} p<0.05$

Implantation may impart a partial recovery of the ON pathway in the $\mathrm{Tg}$ S334ter-3 rat

As described in Chapter III, degeneration in the Tg S334ter-3 rat is much more rapid than the $\mathrm{Tg} \mathrm{P} 23 \mathrm{H}-1$ rat. The $\mathrm{Tg}$ S334ter-3 rat also showed a significantly different ON-OFF index from WT. Even as early as the age of implantation (P35), OFF responses dominated the SC response profile. Although this may be a significant hurdle for a neuroprotective effect, I made comparisons in the Tg S334ter-3 in the same manner as described for the $\mathrm{Tg} \mathrm{P} 23 \mathrm{H}-1$ above. Figure 40 plots the ON-OFF index for regions under the influence of the PVA and regions outside of its influence and shows that despite the more aggressive nature of the $\operatorname{Tg}$ S334ter-3 degeneration that the prosthetic significantly increases the ON-OFF index in regions under its influence. There was no significant difference between sites that are directly adjacent $(<400 \mu \mathrm{m})$ to the area of influence and areas more than $400 \mu \mathrm{m}$ away, similar to the effect seen in 
$\mathrm{Tg} \mathrm{P} 23 \mathrm{H}-1$ rats. When compared to untreated $\mathrm{Tg}$ animals, the ON-OFF index was significantly higher for regions under the influence of the PVA.

\section{S334ter with bPVA Device}

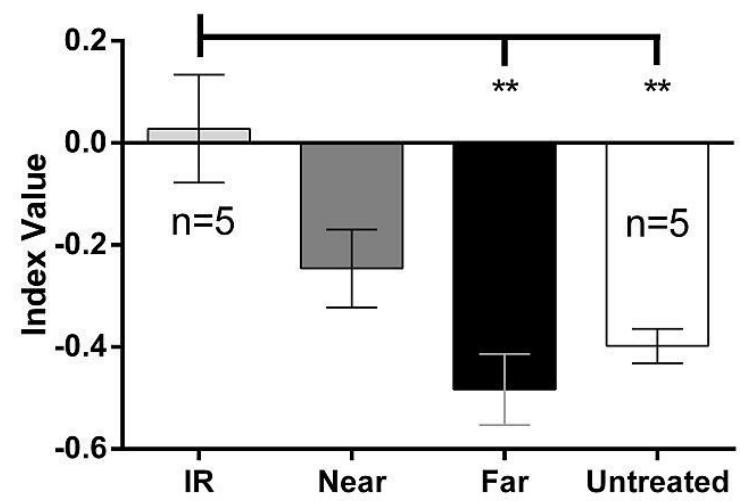

Figure 40. ON-OFF index comparison in relation to area of PVA influence. Regions under the influence of the PVA showed a significant increase in the ON-OFF index, even when compared to untreated Tg rats. ${ }^{* *} p<0.01$

Figure 41 plots the ON and OFF peak responses and response duration for $\mathrm{Tg}$ S334ter-3 rats. Though there was a significant change in the ON-OFF index, there was no significant change in the $\mathrm{ON}$ duration or peak response. There was a trend however, towards a higher ON peak response for sites under the influence of the PVA. This may be a consequence of a low $n$ (5) and may be significant if the number of rats was increased. An important point to make here as well was the lack of change in the OFF pathway as the animals aged, the OFF response was consistent regardless of the measurement used. Surprisingly, the OFF response of the untreated animals was much larger than the OFF response in implanted animals. This explains the significant change in the ON-OFF index when compared to untreated animals. However, when the internal control regions are compared to the IR-responsive regions, there is a trend for an increased ON response in regions under the influence of the PVA and the ON-OFF index value was still significantly higher in regions of PVA influence. 

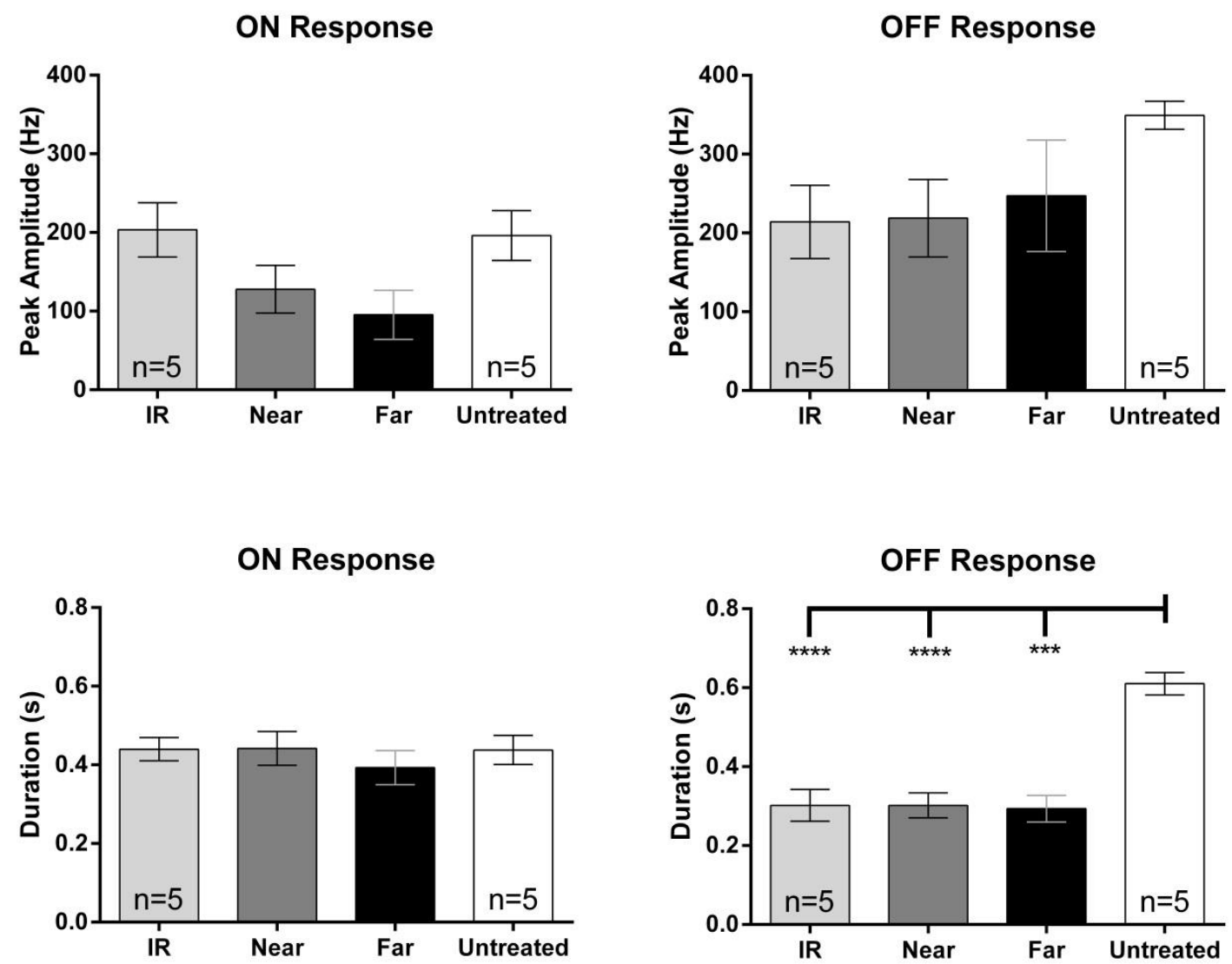

Figure 41.

A. Comparison of the $\mathrm{ON}$ and OFF peak response in relation to regions of PVA influence. No significant changes were found, though a trend towards larger ON responses was seen in regions of PVA influence.

$B$. Comparison of the ON and OFF response duration. The OFF response was significantly longer in untreated Tg animals. ${ }^{\star * \star} p<0.001,{ }^{\star * * *} p<0.0001$

In contrast to the change in $\mathrm{Tg} \mathrm{P} 23 \mathrm{H}-1$ spontaneous activity, there was no significant change in the spontaneous activity in Tg S334ter-3 rats either across regions within an implanted rat or compared to untreated age-matched Tg rats (Figure 42). 


\section{Spontaneous Activity}

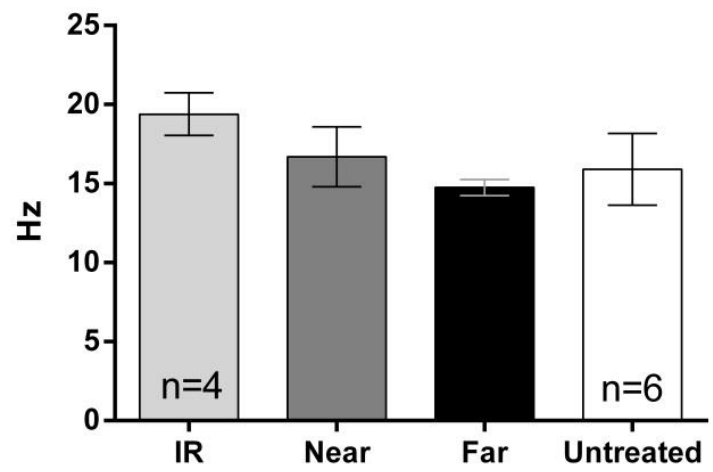

Figure 42. Spontaneous activity in implanted and untreated Tg S334ter-3 rats. No significant change in spontaneous activity was seen.

\section{Discussion}

My data suggest there may be a neuroprotective effect on the function of the retina in regions under the influence of the prosthesis. In both the $\mathrm{Tg} \mathrm{P} 23 \mathrm{H}-1$ and $\mathrm{Tg}$ S334ter-3 rat models show significantly larger ON-OFF indices in regions within IR-evoked responses, suggesting a rescue of function within the ON pathway. Although there are trends that indicate some change with distance, the significant effects appear local to the region of PVA influence. This suggests that the effect is either due to stimulation provided by the device, albeit low in level, or the presence of a foreign object. I favor the former explanation since it is local and not global, which would be expected from a foreign object influence. Rather, the data suggests there is an effect directly related to contact with the PVA. Whether this is a physical interface or functional contact (current spreading to regions away from the PVA) cannot be determined.

My data supports the hypothesis that a prosthetic device can impart a neuroprotective effect by prolonging ON pathway function, especially in the 
slower degenerating $\mathrm{Tg} \mathrm{P} 23 \mathrm{H}-1$ rat. The results in Tg S334ter-3 rats were not conclusive but this may be due to the length of the implantation. In the $\mathrm{Tg} \mathrm{P} 23 \mathrm{H}$ 1 rats, the implant was left in the eye for $\sim 115$ days while in Tg S334ter-3 rats, it was only in the eye for $\sim 40$ days. The effect may simply require a longer duration of implantation than the 40 days used here. This time point was chosen because the degeneration is much more rapid in the $\operatorname{Tg}$ S334ter-3, so it can be a difficult balance finding an age with robust visually-evoked responses and the implantation duration. This may be why there was simply a trend for increased ON pathway function rather than significant difference.

In patients, if implantation enhances the maintenance of retinal function, the long term prognosis for patients, regardless of treatment, will be better. The data suggest it may be beneficial to implant these devices earlier in the degenerative process, as the function of the ON pathway may be spared. Importantly, there was no detrimental effect of the OFF pathway response with implantation in the $\mathrm{Tg} \mathrm{P} 23 \mathrm{H}-1$ rats, leading to the conclusion that there is a general beneficial effect of implantation. While not as compelling, there still was a trend within the ON pathway and a significant increase in the ON-OFF index of IR responsive regions in $\mathrm{Tg}$ S334ter-3 rats. When taken together with the data in Chapter III, the larger ON responses in implanted animals supports the maintenance and/or recovery of the ON pathway, with no discernible latency in the loss of visually evoked responses. This does not preclude the possibility that an effect on the retina still exists after photoreceptors are lost. This could be in the form of preservation of the remaining cellular layers (INL, RGC), receptor 
expression (mGluR, Glycine, GABA), or RPE structure as RPE cells often invade other cellular layers during the degenerative process.

An argument could be made that the presence of the devices actually seemed to be detrimental to the function of the retina based on the comparison to untreated $\mathrm{Tg}$ animals. Indeed, there was no significant difference in any measure between regions under PVA influence when compared to untreated controls. However, this may be explained by light damage during the implantation surgery. Multiple studies have shown that increased exposure to light, even under normal ambient intensities, accelerates the degenerative process in both RP models used here (Organisciak et al., 2003, Vaughan et al., 2003, Jozwick et al., 2006, Thomas et al., 2007). In fact, even an exposure to bright light as short as 1 hour has been reported to significantly accelerate photoreceptor degeneration in the $\mathrm{Tg} \mathrm{P} 23 \mathrm{H}-1$ rat (Vaughan et al 2003). In light of these reports, it is likely that the light used to illuminate the eye during the implantation surgery negatively affected the retina in implanted animals. The surgery typically lasted $\sim 45$ minutes, which is a significant amount of time to have a bright light illuminate the eye. Vaughan et al. (2003) exposed two lines each of Tg P23H-1 and Tg S334ter-3 rats to intense light $\left(1200-1400 \mathrm{~cd} / \mathrm{m}^{2}\right)$ for $1-8$ hours. The light source used for implantation surgery was very bright, somewhere between 1100 and $10,000 \mathrm{~cd} / \mathrm{m}^{2}$, certainly enough light to damage the retina. Unfortunately, the experimental setup did not allow for comparison to the non-implanted (and presumably non-light damaged) eye as the stereotaxic apparatus physically impeded the ability to direct the full field light source on the opposite eye. Bearing 
this in mind, the most effective comparison would be within the same eye, comparing areas under PVA influence to areas outside of its influence. When these regions are compared, $\mathrm{Tg} \mathrm{P} 23 \mathrm{H}-1$ ON pathway function is significantly larger within the PVA influenced area. Although not statistically significant, the trend is the same in Tg S334ter-3 rats.

DeMarco et al (2007) reported trophic effects of implantation of mPVAs in $\mathrm{RCS}$ rats. First, visual responses were more robust in areas under the influence of the mPVA. Secondly, there was an increase in the number of visual responsive SC sites even in regions that were not correlated with the likely position of the prosthesis. This may be due to a more global response to either the surgery or foreign body response to the prosthesis. The global response could be an increase in trophic factors, such as Fgf2 as reported by Ciavatta et al. (2009) in the RCS rat. While an increase in trophic factors cannot be ruled out for my data, there was no observable delay in the loss of visual responses. My data support an alternative hypothesis. Since the effect was localized to area under the influence of the PVA, it is more likely that the current generated by the prosthesis results in the functional neuroprotection. While the devices are not producing enough current to elicit PVA-evoked responses to ambient light, they still produce current in response to ambient light (see Figure 10), albeit at a much reduced level. The rats are housed in a normal 12/12 light-dark cycle, so there are up to 12 hours each day that the devices are producing some basal level of current. In addition, in both $\mathrm{Tg}$ models, the only areas that showed a significant increase in the ON-OFF index were the areas directly under the influence of the 
device. This does not rule out the possibility that the electrical current elicits an increase in trophic factors directly where it sits in the retina, but regardless, the effect was tied to a functional connection with the bPVA. This is consistent with reported neuroprotection using trans-corneal electrical stimulation in RCS rats where there was a preservation of both numbers of photoreceptors and ERG amplitudes in treated animals (Morimoto et al., 2007). It should be noted that the RCS rat and its photoreceptor degeneration is caused by a very different mechanism. Specifically, in the RCS rat the cells of the retinal pigment epithelium are dysfunctional and fail to phagocytize disks shed from the outer segment of photoreceptors. This leads to a buildup of debris in the subretinal space and a cascade of events ending in photoreceptor death. As a consequence, the effect of implantation may have quite different trophic effects.

The important question remains, however, what is the underlying cause of the neuroprotective effect? Does electrical stimulation cause an increase in trophic factors and if so, which ones? Does electrical current act directly on BCs to change their membrane potential and if so, does it close or open ion channels and which ones (excitatory at the dendritic or axonal tips or inhibitory at the axonal tips)? If the hypothesis for the loss of the ON pathway described in Chapter III is correct, then I believe my results support the theory that electrical current acts directly on the retina, likely hyperpolarizing BCs through some mechanism. This could prevent a reactive (excitotoxic or other) mechanism that reduces the expression of mGluR6. Regardless, the data suggest that the implant itself prevents the loss of the ON pathway in both of these models of RP. 


\section{CHAPTER V}

\section{SUMMARY AND FUTURE DIRECTIONS}

In summary, Chapter II described the retina-prosthesis interface with the following conclusions:

1. Targeted prosthesis stimulation yields a high success rate for retinal activation.

2. Synaptic blocker injections show PVA-evoked responses are mediated through INL neurons rather than through direct activation of RGCs.

3. Activation in the SC is generally correlated to the location of the device in the eye.

4. Implantation age and duration had no effect on threshold for stimulation.

5. Pixel size in bPVA devices did not affect threshold but bPVA devices had a significantly higher threshold for activation than mPVA devices.

6. All thresholds for activation were below established safety limits for the retina.

7. A linear range of increasing PVA-mediated responses was present.

8. Focal activation of the retina was possible with bPVA devices but not mPVA devices.

9. The bPVA device design represents a significant improvement in prosthesis design of the mPVA design. 
One of the major hurdles to treating RP is the heterogeneity in the cause of the disease. The cost for a company to develop genetic treatments for every cause of RP is not cost effective for these companies. More comprehensive treatments that are independent of the cause are most likely to be developed on a commercial basis. To this end, the use of prosthetic devices, stem cells, and optogenetic tools likely represent the most promising options. When safety concerns are considered, currently prosthetic devices have been deemed safe by the FDA while both stem cells and optogenetic manipulation still have concerns and questions that have yet to be addressed. In addition, the state of the retina may guide which treatments will be most effective for a given patient. If BCs are unable to faithfully transmit signals mediated by glutamate, prosthetic devices will still work at either the BC or RGCs level.

The Argus II system developed by Second Sight, Inc. is an epiretinal design that is the only retinal prosthesis to be approved by the FDA. It has a proven track record with years of research to back up its safety and efficacy. However, the best resolution achieved with this device is quite poor, only reaching 20/1260. The prosthesis group in Germany led by Dr. Zrenner has achieved a much higher resolution (20/560) with their subretinal implant. However, both systems rely on an external power supply. Both of these issues are addressed by the bPVA design. It is a subretinal chip that has the potential for high acuity vision while being completely self-contained. The debate between subretinal and epiretinal prosthetic designs is still ongoing with both systems 
having their advantages and disadvantages. In my opinion, subretinal stimulation using a photovoltaic array offers the best design for a retinal prosthetic device.

The future of the retinal prosthesis field will undoubtedly be higher resolution designs. A thorough understanding of what vision is possible with visual prosthetics will also drive future designs and stimulus protocols. While forms, luminance and rudimentary motion can be encoded by current designs, the visual system encodes much more information than these basic perceptions. Can other facets of vision be faithfully encoded such as color vision, depth perception, and ON/OFF signals? Regardless of this outcome to these questions, prosthetic devices have been proven safe, can transmit information to completely blind patients, and have long term stability.

Chapter III described functional changes associated with photoreceptor degeneration and concluded the following:

1. In the $\mathrm{Tg} \mathrm{P} 23 \mathrm{H}-1$ rat model of $\mathrm{RP}$, there is a shift in $\mathrm{SC}$ responses from $\mathrm{ON}$ dominated to OFF dominated that increases as a function of age.

2. In the Tg S334ter-3 rat model of RP, SC responses are strongly OFF dominated from a very early age likely due to the rapid nature of this model's degeneration.

3. MEA recordings in $\mathrm{Tg} \mathrm{P} 23 \mathrm{H}-1$ rat retina indicate that the progressive changes are caused by the loss of $\mathrm{ON}$ signaling within the retina rather than changes within the SC. 
4. An increase in spontaneous activity and rhythmic activity in Tg P23H-1 RGCs are only seen in OFF and NR RGCs once photoreceptors have begun to degenerate.

The functional consequences of photoreceptor degeneration have not been extensively explored. The state of the retina during the degenerative process may have profound effects on the potential for certain treatments to be effective. If we can categorize these changes, future research can work to either prevent or reverse these changes and better treat patients with degenerative diseases such as RP and AMD. In addition, studying the reactive changes in the retina may be beneficial towards understanding plasticity within the retina. Other species, such as chickens (Fisher, 2005) and frogs (Filoni, 2009), are able to regenerate cells within the retina if they are damaged. Some of the processes that occur during the degenerative process may be common to proliferative or developing cells. In this regard, studying the mechanisms of these reactive changes may help discover why mammalian retinas (and neurons in general) are unable to regenerate and how plasticity in the retina may be encouraged and utilized.

Chapter IV described a possible functional neuroprotective mechanism through implantation of a prosthetic device with the following conclusions: 1. Implantation of bPVA devices significantly increased the function of the ON pathway in $\mathrm{Tg} \mathrm{P} 23 \mathrm{H}-1$ rats. 
2. The effect was present only in SC regions that were under the influence of the PVA.

3. Implantation in Tg S334ter-3 rats showed a similar trend for an increased ON response but only was significantly different when analyzing the ON-OFF index.

Electrical stimulation of the retina may be an effective neuroprotective mechanism, whether applied directly to the retina or by stimulating the whole eye. The functional recovery described in this chapter was only present in regions under the influence of the PVA. This suggests that this example of functional neuroprotection may not be possible using external stimulation of the retina. If the current was increased, this may be enough to see a similar effect. The results with the $\mathrm{Tg}$ S334ter-3 rats were not as strong as in implanted $\mathrm{Tg}$ $\mathrm{P} 23 \mathrm{H}-1$ rats, but as previously discussed, this may be due to the difference in implantation duration. Alternatively, the rapid degeneration and loss of the ON pathway in the Tg S334ter-3 may prevent electrical stimulation from being effective in this model. For the majority of RP patients, this is a slow process with many patients not being severely impaired until late in life. As such, the $\mathrm{Tg} \mathrm{P} 23 \mathrm{H}-$ 1 rat probably represents a more analogous model of RP than the Tg S334ter-3. If the effect is truly mediated by the current generated by the device, the next logical step would be to actively stimulate the device with IR light over the course of a few weeks (or months) to see if the effect is related to the amount of current generated by the PVA. This may enhance the effect and increase the ON response even further. In addition, even if the progression of the disease is not slowed or halted, if the retinal circuitry is spared during the degenerative process, 
such as the expression of mGluR6 on BCs, this can increase the treatment options and effectiveness for a patient. 


\section{REFERENCES}

Ball, S., Pardue, M.T., McCall, M.A., Gregg, R.G., and Peachey, N.S. (2003) Immunohistochemical analysis of the outer plexiform later in the nob mouse shows no abnormalities. Visual Neuroscience 20:267-272.

Baylor, D.A. and Fettiplace, R. (1977) Kinetics of synaptic transfer from receptors to ganglion cells in turtle retina. J Physiology 271(2): 425-448.

Baylor, D.A., Lamb, T.D., and Yau, K.W. (1979) Responses of retinal rods to single photons. J Physiology 288, 613-634.

Berntson, A. and Taylor, W.R. (2000) Response characteristics and receptive field widths of on-bipolar cells in the mouse retina. J Physiology 524(3), 879-889.

Bijveld., M.M., Florijn, R.J., Bergen, A.A., van den Born, L.I., Kammermans, M., Prick, L., Riemslag, F.C., van Schooneveld, M.J., Kappers, A.M., and van Genderen, M.M. (2013) Genotype and Phenotype of 101 Dutch Patients with Congenital Stationary Night Blindness. Ophthalmology (ahead of print).

Butterwick, A., Vankov, A., Huie, P., Freyvert, Y., and Palanker, D. (2007) Tissue damage by pulsed electrical stimulation. IEEE Trans Biomed Eng 54(12), 22612267.

Cai, C., Ren, Q., Desai, N.J., Rizzo, J.F., Fried, S.I. (2011) Response variability to high rates of electric stimulation in retinal ganglion cells. J. Neurophysiology 106: 153-162.

Chalupa, L.M. and Werner, J.S. (2003) The Visual Neurosciences. 1930 pp.

Chan, L.H., Lee, E-J., Humayun, M.S., and Weiland, J.D. (2011) Both electrical stimulation thresholds and SMI-32_immunoreactive retinal ganglion cell density correlate with age in S334ter line 3 rat retina. J. Neurophysiol 105: 2687-2697

Chow, A.Y., Chow, V.Y., Packo, K.H., Pollack, J.S., Peyman, G.A., and Schuchard, R. (2004) The artificial silicon retina microchip for the treatment of vision loss from retinitis pigmentosa. Arch Ophthalmol 122(4), 460-469.

Chow, A.Y., Bittner, A.K., and Pardue, M.T. (2010) The Artificial Silicon Retina in retinitis pigmentosa patients (an American Opthalmological Association thesis). Trans Am Ophthalmol Soc. 108, 120-154. 
Ciavatta, V.T., Kim, M., Wong, P., Nickerson, J.M., Schuler, R.K., McLean, G.Y., and Pardue, M.T. (2009) Retinal Expression of Fgf2 in RCS rats with Subretinal Microphotodiode Array. Invest Ophthal and Vis Sci 50(10): 4523-4530.

Cirone, J., Pothecary, C.A., Turner, J.P., and Salt T.E. (2002) Group I metabotropic glutamate receptors (mGluRs) modulate visual responses in the superficial superior colliculus of the rat. J Physiology 541(3), 895-903.

Concepcion, F., Mendez, A.M., and Chen, J. (2002) The carboxyl-terminal domain is essential for rhodopsin transport in rod photoreceptors. Vision Research 42, 417-426.

Cuenca, N., Pinilla, I., Sauve, Y., Lu, B., Wang, S., and Lund, R.D. (2004) Regressive and reactive changes in the connectivity patterns of rod and cone pathways of $\mathrm{P} 23 \mathrm{H}$ transgenic rat retina. Neuroscience 127, 301-317.

Danias, J., Shen, F., Goldblum, D., Chen, B., Ramos-Esteba, J., Podos, S.M., and Mittag, T. (2002) Cytoarchitecture of the retinal ganglion cells in the rat. Inv Ophth and Vis Sci 43(3), 587-594.

DeMarco, P.J. Jr., Yarbrough, G.L., Yee, C.W., McLean, G.Y., Sagdullaev, B.T., Ball, S.L., and McCall, M.A. (2007) Stimulation via a subretinally placed prosthetic elicits central activity and induces a trophic effect on visual responses. Inv Ophth and Vis Sci 48(2), 916-926.

Demas, J., Sagdullaev, B.T., Green, E., Jaubert-Miazza, L., McCall, M.A., Gregg, R.G., Wong, R.O.L., and Guido, W. (2006) Failure to Maintain Eye-Specific Segregation in nob, a Mutant with Abnormally Patterned Retinal Activity. Neuron 50: 247-259.

Drager, U.C. and Hubel, D.H. (1976) Topography of Visual and Somatosensory Projections to Mouse Superior Colliculus. J Neuropphysiology 39(1), 91-101.

Dryja, T.P., McGee, T.L., Reichel, E., Hahn, L.B., Cowley, G.S., Yandell, D.W., Sandberg, M.A., and Berson, E.L. (1990) A point mutation of the rhodopsin gene in one form of retinitis pigmentosa. Nature 343(6256):364-366.

Eikenscheidt, M., Jenkner, M., Thewes, R., Fromherz, P., and Zeck, G. (2012) Electrical stimulation of retinal neurons in epiretinal and subretinal configuration using a multicapacitor array. J. Neuropysiol 107: 2742-2744.

Filoni, S. (2009) Retina and lens regeneration in anuran amphibians. Seminars in Cell and Developmental Biology 20(5): 528-534. 
Fishman, G.A., Farber, M.D., and Derlacki, D.J. (1988) X-linked retinitis pigmentosa: profile of clinical findings. Arch Ophthalmol 106: 369-75.

Fisher, A.J. (2005) Neural regeneration in chick retina. Progress in Retina and Eye Research 24(2): 161-182.

Freeman, D.K. and Fried, S.I. (2011) Multiple components of ganglion cell desensitization in response to prosthetic stimulation. J. Neural Engineering 8(1): 016008

Friedman, D.S., O'Colmain, B.J., Munoz, B., Tomany, S.C., McCarty, C., de Jong, P.T., Nemesure, B., Mitchell, P., and Kempen, J. (2004) Prevalence of age-related macular degeneration in the United States. Archives of Opthalmology 122(4), 564-572.

Gebhard, J.W. (1952) Thresholds of the human eye for electric stimulation by different wave forms. J Experimental Psychology 44(2), 132-140.

Hartong, D.T., Berson, E.L., and Dryja, T.P. (2006) Retinitis Pigmentosa. The Lancet 368(9549), 1795-1809.

Hoffman, K.P. (1973) Conduction velocity in pathways from retina to superior colliculus in the cat: a correlation with receptive-field properties. J Neurophysiol 36, 409-424.

Humayun, M.S., de Juan, E. Jr., Weiland, J.D., Dagnelie, G., Katona, S., Greenberg, R., and Suzuki, S. (1999) Pattern electrical stimulation of the human retina. Vision Research 39(15): 2569-2576.

Humayun, M.S., Dorn, J.D., da Cruz, L., Dagnelie, G., Sahel, J.A., Stanga, P.E., Cideciyan, A.V., Duncan, J.L., Eliott, D., Filley,E., Ho, A.C., Santos, A., Safran, A.B., Arditi, A., Del Priore, L.V., Greenberg, R.J.; Argus II Study Group. (2012) Interim results from the international trial of Second Sight's visual prosthesis. Ophthalmology. 119(4):779-88.

Illing, M.E., Rahul, R.S., Bence, N.F., and Kopito, R.R. (2002) A rhodopsin mutant linked to autosomal dominant retinitis pigmentosa is prone to aggregate and interacts with the ubiquitin proteasome system. J Biolog Chem 277(37) 34150-34160.

Jacobs, G.H., Neitz, J., and Deegan, J.F. (1991) Retinal receptors in rodents maximally sensitive to ultraviolet light. Nature Letters 353:655-656.

Jacobs, G.H., Fenwick, J.A., and Williams, G.A. (2001) Cone-based vision of rats for ultraviolet and visible lights. J Exp Biology 204, 2439-2446. 
Jensen, R.J. and Rizzo, J.F. (2008) Activation of retinal ganglion cells in wildtype and rd1 mice through electrical stimulation of the retinal neural network. Vision Research 48, 1562-1568.

Jensen, R.J., and Rizzo, J.F. (2009) Activation of ganglion cells in wild-type and rd1 mouse retinas with monophasic and biphasic current pulses. J Neural Eng (3): 035004.

Jozwick, C., Valter, K., and Stone, J. (2006) Reversal of functional loss in the $\mathrm{P} 23 \mathrm{H}-3$ rat retina by management of ambient light. Experimental Eye Research 83: 1074-1080.

Kondo, M., Sakai, T., Komeima, K., Kurimoto, Y., Ueno, S., Nishizawa, Y., Usukura, J., Fujikado, T., Tano, Y., and Terasaki, H. (2009) Generation of a transgenic rabbit model of retinal degeneration. Invest Ophth and Vis Sci 50(3), 1371-1377.

Kolomiets, B., Dubus, E., Simonutti, M., Rosolen, S., Sahel, J.A., and Picaud, S. (2010) Late histological and functional changes in the $\mathrm{P} 23 \mathrm{H}$ rat retina after photoreceptor loss. Neurobiology of Disease 38: 47-58.

Kurosawa, T. (1954) Selective stimulation of retinal receptors with electric currents of various forms. Tohoku Journal of Experimental Medicine 62(2), 195207.

Kimberling, W.J., Weston, M.D., Möller, C., Davenport, S.L., Shugart, Y.Y., Priluck, I.A., Martini, A., Milani, M., and Smith, R.J. (1990) Localization of Usher syndrome type II to chromosome 1q. Genomics. 7(2):245-249.

Lewin, A.S., Drenser, K.A., Hauswirth, W.W., Nishikawa, S., Yasumura, D., Flannery, J.G., and LaVail, M.M. (1998) Ribozyme rescue of photoreceptor cells in a transgenic rat model of autosomal dominant retinitis pigmentosa. Nat Med 4(8), 1081.

Liu, C., Li, Y., Peng, M., Laties, A.M., and Wen, R. (1999) Activation of caspase3 in the retina of transgenic rats with the rhodopsin mutation S334ter during photoreceptor degeneration. J Neuroscience 19(12), 4778-4785.

Lucas, P.W., Dominy, N.J., Riba-Hernandez, P., Stoner, K.E., Yamashita, N., Loria-Calderon, E., Petersen-Pererira, W., Rojas-Duran, Y., Salas-Pena, R., Solis-Madrigal, S., Osorio, D., and Darvell, B.W. (2003) Evolution and Function of Routine Trichromatic Vision in Primates. Evolution 57(11): 2636-2643.

Lund, R.D. (1964) Terminal distribution in the superior colliculus of fibres originating in the visual cortex. Nature 204, 1283-1285. 
Manookin, M.B., Beaudoin, D.L., Ernst, Z.R., Flagel, L.J., and Demb, J.B. Disinhibition combines with excitation to extend the operating range of the OFF visual pathway in daylight J Neuroscience 28(16), 4136-4150.

Marc, R.E. and Jones B.W. (2003) Retinal remodeling in inherited photoreceptor degenerations. Molecular Neurobiology 28(2), 139-147.

Margolis, D.J., Newkirk, G., Euler, T., and Detwiler, P.B. (2008) Functional stability of retinal ganglion cells after degeneration-induced changes in synaptic input. J Neuroscience 28(25), 6526-6536.

Mathieson, K., Loudin, J., Goetz, G., Huie, P., Wang, L., Kamins, T.I., Glambos, L., Smith, R., Harris, J.S., Sher, A., and Palanker, D. (2012) Photovoltaic Retinal Prosthesis with High Pixel Density. Nature Photonics 6(6) 391-397.

May, P.J. (2006) The mammalian superior colliculus: laminar structure and connections. Prog in Brain Res 151, 321-378.

Maynard, E.M., Nordhausen, C.T., and Normann, R.A. (1997) The Utah intracortical electrode array: a recording structure for potential brain-computer interfaces. Annual Rev Biomed Eng 3, 145-168.

McNally, N., Kenna, P.F., Rancourt, D., Ahmed, T., Stitt, A., Colledge, W.H., Lloyd, D.G., Palfi, A., O'Neill, B., Huphries, M.M., Humphries, P., and Farrar, G.J. (2002) Murine model of autosomal dominant retinitis pigmentosa generated by targeted deletion at codon 307 of the rds-peripherin gene. Human Molec Gen 11(9), 1005-1016.

McNaughtan, P.A. (1990) Light Response of Vertebrate Photoreceptors. Physiological Reviews 70(3): 847-883.

Mocko, J.A., Kim, M., Faulkner, A.E., Cao, Y., Ciavatta, V.T., and Pardue, M.T. (2011) Effects of subretinal electrical stimulation in Mer-KO mice. Inv Ophth and Vis Sci 52(7), 4223-4230.

Morimoto, T., Miyoshi, T., Fujikado, T., Tano, Y., and Fukuda, Y. (2005) Electrical stimulation enhances the survival of axotomized retinal ganglion cells in vivo. Invest Ophthalmol \& Vis Sci. 46(6):2147-2155.

Moimoto, T., Fujikado, T.,Choi, S., Kanda, Hiroyuki, K., Miyoshi, T., Fukuda, Y., and Tano, Y. (2007) Transcorneal Electrical Stimulation Promotes the Survival of Photoreceptors and Preserves Retinal Function in Royal College of Surgeons Rats. Invest Ophthalmol \& Vis Sci 48(10): 4725-4732.

Morimoto, T., Kanda, H., Kondo, M., Terasaki, H., Nishida, K., and Fujikado, T. (2012) Transcorneal electrical stimulation promotes survival of photoreceptors 
and improves retinal function in rhodopsin P347L transgenic rabbits. Invest Ophthalmol \& Vis Sci. 53(7):4254-4261.

Ni, Y.Q., Gan, D.K., Xu, H.D., Xu, G.Z., Da, C.D. (2009) Neuroprotective effect of transcorneal electrical stimulation on light-induced photoreceptor degeneration. Exp Neurol. 219(2):439-452.

O'Hearn, T.M., Sadda, S.R., Weiland, J.D., Maia, M., Margalit, E., and Humayun, M.S. (2006) Electrical stimulation in normal and retinal degeneration (rd1) isolated mouse retina. Vision Research 46: 3198-3204.

Olsson, J.E., Gordon, J.W., Pawlyk, B.S., Roof, D., Hayes, A., Molday, R.S., Mukai, S., Cowley, G.S., Berson, E.L., and Dryja, T.P. (1992) Transgenic mice with a rhodopsin mutation (Pro23His): a mouse model of autosomal dominant retinitis pigmentosa. Neuron 9(5), 815-830.

Organisciak, D.T., Darrow, R.M., Barsalou, L., Kutty, R.K., and Wiggert, B. (2003) Susceptibility to Retinal Light Damage in Transgenic Rats with Rhodopsin Mutations. Invest Oph Vis Sci 44(2): 486-492.

Palanker, D., Vankov, A., Huie, P., and Baccus, S. (2005) Design of a highresolution optoelectronic retinal prosthesis. J Neural Eng. 2: S105-S120.

Pardue, M.T., Stubbs, E.B. Jr, Perlman, J.I., Narfström, K., Chow, A.Y., and Peachey, N.S. (2001) Immunohistochemical studies of the retina following longterm implantation with subretinal microphotodiode arrays. Exp Eye Res.

73(3):333-343.

Pardue, M.T., Phillips, M.J., Yin, H., Sippy, B.D., Webb-Wood, S., Chow, A.Y., and Ball, S.L. (2005) Neuroprotective effect of subretinal implants in the RCS rat. Invest Ophthalmol \& Vis Sci 46(2), 674-682.

Petters R.M., Alexander, C.A., Wells, K.D., Collins, K.D., Sommer, J.R., Blanton M.R., Rojas, G., Hao, Y., Flowers, W.L., Banin, E., Cideciyan, A.V., Jacobson, S.G., and Wong, F. (1997) Genetically engineered large animal model for studying cone photoreceptor survival and degeneration in retinitis pigmentosa. Nat Biotechnol. 15, 965-970.

Pothecary, C.A., Thompson, H., and Salt, T.E. (2005) Changes in glutamate receptor function in synaptic input to the superficial superior colliculus (SSC) with aging and in retinal degeneration in the Royal College of Surgeons (RCS) rat. Neurobiology of Aging 26: 965-972.

Price, B.A., Sandoval, I.M., Chan, F., Simons, D.L., Wu, S.M., Wensel,T.G., and Wilson, J.H. (2011) Mislocalization and Degradation of Human P23H-Rhodopsin- 
GFP in a Knockin Mouse Model of Retinitis Pigmentosa Invest Ophthalmol \& Vis Sci. 52(13):9728-9736.

$\mathrm{Pu}, \mathrm{M}$., Xu, Liand, and Zhang, H. (2006) Visual Response Properties of Retinal Ganglion Cells in the Royal College of Surgeons Dystrophic Rat Invest Ophthalmol \& Vis Sci. 47(8): 3579-3585.

Purves, D., Augustine, G.J., Fitzpatrick, D., Katz, L.C., LaMantia, AS., McNamara, J.O., and Williams, S.M. (2001) Neuroscience $2^{\text {nd }}$ edition. 681

Puthussery, T., Gayet-Primo, J., Pandey, S., Duoisin, R.M., and Taylor, W.R. (2009) Differential loss and preservation of glutamate receptor function in bipolar cells in the rd10 mouse model of retinitis pigmentosa. Euro $\mathrm{J}$ of Neurosci 29: 1533-1542.

Rattay, F. and Resatz, S. Effective Electrode Configuration for Selective Stimulation with Inner Eye Prostheses. IEEE Trans Biomed Eng 51(9), 16591664.

Renner, A.B., Kellner, U., Cropp, E., and Foerster, M.H. (2006) Dysfunction of transmission in the inner retina: incidence and clinical causes of negative electroretinogram. Graef's Archive for Clinical and Exp. Ophthalmol. 244(11): 1467-1473.

Robinson, D.A. (1972) Eye movements evoked by collicular stimulation in the alert monkey. Vision Research 12 (11), 1795-1808.

Ross, J.W., Fernandez de Castro, J.P., Zhao, J., Samuel, M., Walters, E., Rios, C., Bray-Ward, P., Jones, B.W., Marc, R.E., Wang, W., Zhou, L., Noel, J.M., McCall, M.A., DeMarco, P.J., Prather, R.S., and Kaplan, H.J. (2012) Generation of an inbred miniature pig model of retinitis pigmentosa. Invest Oph Vis Sci 53(1), 501-507.

Sauve, Y., Girman, S.V., Wang, S., Lawrence, J.M., and Lund, R.D. (2001) Progressive Visual Sensitivity Loss in the Royal College of Surgeons Rat: Perimetric Study in the Superior Colliculus. Neuroscience 103(1): 51-63.

Schmeer,C.W., Wohl, S.G., and Isenmann, S. (2012) Cell-replacement therapy and neural repair in the retina. Cell Tissue Res 349: 363-374.

Sekirnjak, C., Hottowy, P., Sher, A., Dabrowski, W., Litke, A.M., and Chichilnisky, E.J. (2008) High-resolution electrical stimulation of primate retina for epiretinal implant design. Neurobiology of Disease 28(17), 4446-4456.

Sekirnjak, C., Hulse, C., Jepson, L.H., Hottowy, P., Sher, A., Dabrowski, W., Litke, A.M., and Chichilnisky, E.J. (2009) Loss of responses to visual but not 
electrical stimulation in ganglion cells of rats with severe photoreceptor degeneration. J Neurophysiol 102, 3260-3269.

Sekirnjak, C., Jepson, L.H., Hottowy, P., Sher, A., Dabrowski, W., Litke, A.M., and Chichilnisky, E.J. (2011) Changes in physiological properties of rat ganglion cells during retinal degeneration. J Neurophysiol 105 (5), 2560-2571.

Shah, H.A., Montezuma, S.R., and Rizzo, J.F. (2006) In vivo electrical stimulation of rabbit retina: Effect of stimulus duration and electrical field orientation. Exp Eye Research 83: 247-254.

Shinde, V.M., Sizova, O.S., Lin, J.H., LaVail, M.M., and Gorbatyuk, M.S. (2012) ER stress in retinal degeneration in S334ter Rho rats. PLoS One 7(3), e33266.

Shyu, JS., Maia, M., Weiland, J.D., O'Hearn, T., Chen, SJ., Margalit, E., Suzuki, S., and Humayun, M.S. (2006) Electrical stimulation in isolated rabbit retina. IEEE Trans on Neural Sys and Rehab Eng 14(3), 290-298.

Siminoff, R., Schwassmann, H.O., and Kruger, L. (1966) An Electrophysiological Study of the Visual Projection to the Superior Colliculus of the Rat. J Comp Neurology 127(4):435-444.

Skorupski, P. and Chittka, L. (2010) Photoreceptor Spectral Sensitivity in the Bumblebee, Bombus impatiens (Hymenoptera: Apidae). PLoS One 5(8): e12049.

Stasheff, E. (2008) Emergence of sustained spontaneous hyperactivity and temporary preservation of OFF responses in ganglion cells of the retinal degeneration (rd1) mouse. J Neurophysiol 99 (3), 1408-1421.

Stasheff, S.F., Shankar, M., and Andrews, M.P. (2011a) Developmental time course distinguishes changes in spontaneous and light-evoked retinal ganglion cell activity in rd1 and rd10 mice. J Neurophysiol 105 (6), 3002-3009.

Strettoi, E., Porciatti, V., Falsini, B., Pignatelli, V., and Rossi, C. (2002) Morphological and functional abnormalities in the inner retina of the $\mathrm{rd} / \mathrm{rd}$ mouse. J Neuroscience 22 (13), 5492-5504.

Suzuki, D., Irieda, H., Homma, M., Kawagishi, I., and Sudo, Y. (2010) Phototactic and Chemotactic Signal Transduction by Transmembrane Receptors and Transducers in Microorganisms. Sensors 10: 4010-4039.

Thomas, B.T., Seiler, M.J., Aramant, R.B., Samant, D., Qiu, G., Vyas, N., Arai, S., Chen, Z., and Sadda, S.R. (2007) Visual Functional Effects of Constant Blue Light in Retinal Degenerate Rat Model. Photochemistry and Photobiology 83: 759-765. 
Thompson, D.A., McHenry, C.L., Li, Y. (2002) Retinal dystrophy due to paternal isodisomy for chromosome 1 or chromosome 2, with homoallelism for mutations in RPE65 or MERTK, respectively. Am J Hum Genet 70: 224-29.

Vaughan, D.K., Coulibaly, S.F., Darrow, R.M., and Organisciak, D.T. (2003) A Morphometric Study of Light-Induced Damage in Transgenic Rat Models of Retinitis Pigmentosa. Invest Oph Vis Sci 44(2): 848-855.

Veraart, C., Raftopoulos, C., Mortimer, J.T., Delbeke, J., Pins, D., Michaux, G., Vanlierde, A., Parrini, S., and Wanet-Defalque, M.C. (1998) Visual sensations produced by optic nerve stimulation using an implanted self-sizing spiral cuff electrode. Brain Research 813(1), 181-186.

Vugler, A.A., and Coffey, P.J. (2003) Loss of calretinin immunoreactive fibers in subcortical visual recipient structures of the RCS dystrophic rat. Experimental Neurology 184(1): 464-478.

Wang, L., Mathieson, K., Kamins, T.I., Loudin, J.D., Galambos, L., Goetz, G., Sher, A., Mandel, Y., Huie, P., Lavinsky, D., Harris, J.S., and Palanker, D.V. (2012) Photovoltaic retinal prosthesis: implant fabrication and performance. J. Neural Engineering 9(4), 046014.

Wang, Y., Weick, M., and Demb, J.B. (2011) Spectral and temporal sensitivity of cone-mediated responses in mouse retinal ganglion cells. J Neurosci 31(21), 7670-7681. 


\title{
CURRICULUM VITAE
}

\author{
James W. Fransen, Ph.D. \\ 2525 Glenmary Ave Apt. 6 \\ Louisville, KY 40204 \\ iwfran04@gmail.com \\ 815-262-3456
}

\section{Education:}

University of Louisville, Louisville, KY (Ph.D. June 2013)

- Department of Anatomical Sciences and Neurobiology

Northern Illinois University (M.S. May 2009)

- Biology Department with Specialization in Anatomical Sciences

University of Illinois (B.S. May 2004)

- Biology Department

\section{Teaching Positions}

- Teaching fellowship

- Human Anatomy and Physiology, Northern Illinois University (Fall 2008-Spring 2009)

- Teaching assistant

- Medical Neuroanatomy

University of Louisville (Fall 2010)

\section{Graduate Fellowship}

- Integrated Programs in Biomedical Sciences (IPIBS) Fellowship University of Louisville (Fall 2009 - Fall 2011)

\section{Post-Doctoral Fellowship}

- NIH Training Grant (T32) through the Institute for Cellular Therapeutics University of Louisville (July 2013 - June 2014) 


\section{Abstracts}

- James W. Fransen, Alex A. Thomas, Heiko Schmid, Maureen A. McCall

"Functional assessment of retinal input to the superior colliculus in the transgenic $\mathrm{P} 23 \mathrm{H}-1$ rat model of retinitis pigmentosa."

Society for Neuroscience, Nov 2011. Washington, D.C.

- James W. Fransen, Moon K. Kim, Alice Adkins, James Loudin, Machelle T. Pardue, Daniel V. Palanker, Maureen A. McCall

"Subretinal implantation of Microphotodiode Arrays: In vivo assessment of threshold and spatial activation."

Association for Research in Vision Ophthalmology (ARVO), May 6-9, 2012 Ft. Lauderdale, FL

- James W. Fransen, Alex A. Thomas, Heiko Schmid, Gobinda Pangeni, Maureen A. McCall

"Response modifications in the superior colliculus result from retinal degeneration in rhodopsin rat models of retinitis pigmentosa."

FASEB Retinal Neurobiology and Visual Processing, July 29-Aug 4, 2012 Steamboat Springs, CO

- James W. Fransen, Gobinda Pangeni, Benjamin M. James, James Loudin, Keith Mathieson, Theodore I. Kamins, James Harris, Daniel V. Palanker, Machelle T. Pardue, Maureen A. McCall

"Responses evoked by monopolar and bipolar photovoltaic microphotodiode arrays in the S334ter-3 rat model of retinitis pigmentosa."

Association for Research in Vision Ophthalmology (ARVO), May 5-9, 2013 Seattle, WA

- Jacob G. Light, James W. Fransen, Alice Adkins, Gobinda Pangeni, James Loudin, Keith Mathieson, Daniel V. Palanker, Maureen A. McCall, Machelle T. Pardue 
"Immunohistochemical and electrophysiological analysis of rat retinas after subretinal implantation of photovoltaic arrays."

Association for Research in Vision Ophthalmology (ARVO), May 5-9, 2013 Seattle, WA

- Juan P. Fernandez de Castro, Jay Demas, James W. Fransen, Paul DeMarco, Henry Kaplan, Maureen McCall

"Changes in retinal ganglion cell activity in the $\mathrm{P} 23 \mathrm{H}$ mini-swine model of retinal degeneration."

Association for Research in Vision Ophthalmology (ARVO), May 5-9, 2013 Seattle, WA 